\title{
Dissipation and Extra Light in Galactic Nuclei. I. Gas\#Rich Merger Remnants
}

\section{Citation}

Hopkins, Philip F., Lars Hernquist, Thomas J. Cox, Suvendra N. Dutta, and Barry Rothberg. 2008. "Dissipation and Extra Light in Galactic Nuclei. I. Gas\#Rich Merger Remnants." The Astrophysical Journal 679 (1): 156-81. https://doi.org/10.1086/587544.

\section{Permanent link}

http://nrs.harvard.edu/urn-3:HUL.InstRepos:41381610

\section{Terms of Use}

This article was downloaded from Harvard University's DASH repository, and is made available under the terms and conditions applicable to Open Access Policy Articles, as set forth at http:// nrs.harvard.edu/urn-3:HUL.InstRepos:dash.current.terms-of-use\#OAP

\section{Share Your Story}

The Harvard community has made this article openly available.

Please share how this access benefits you. Submit a story.

Accessibility 
Submitted to ApJ, OCTOBER 24, 2007

Preprint typeset using LTEX style emulateapj v. 08/22/09

\title{
DISSIPATION AND EXTRA LIGHT IN GALACTIC NUCLEI: I. GAS-RICH MERGER REMNANTS
}

\author{
Philip F. Hopkins ${ }^{1}$, LARs Hernquist ${ }^{1}$, Thomas J. CoX ${ }^{1,2}$, Suvendra N. DutTa ${ }^{1}$, \& BARry RothberG ${ }^{3,4}$ \\ Submitted to ApJ, October 24, 2007
}

\begin{abstract}
We study the origin and properties of "extra" or "excess" central light in the surface brightness profiles of remnants of gas-rich mergers. By combining a large set of hydrodynamical simulations with data on observed mergers that span a broad range of profiles at various masses and degrees of relaxation, we show how to robustly separate the physically meaningful extra light - i.e. the stellar population formed in a compact central starburst during a gas-rich merger - from the outer profile established by violent relaxation acting on stars already present in the progenitor galaxies prior to the final stages of the merger. This separation is sensitive to the treatment of the profile, and we demonstrate that certain fitting procedures can yield physically misleading results. We show that our method reliably recovers the younger starburst population, and examine how the properties and mass of this component scale with e.g. the mass, gas content, and other aspects of the progenitors. We consider the time evolution of the profiles in different bands, and estimate the biases introduced by observational studies at different phases and wavelengths. We show that, when appropriately quantified, extra light is ubiquitous in both observed and simulated gas-rich merger remnants, with sufficient mass $(\sim 3-30 \%$ of the stellar mass $)$ to explain the apparent discrepancy in the maximum phase-space densities of ellipticals and their progenitor spirals. The nature of this central component provides a powerful new constraint on the formation histories of observed systems and can inform both our studies of their progenitors and our understanding of the global kinematics and structure of spheroids.
\end{abstract}

Subject headings: quasars: general — galaxies: nuclei — galaxies: active — galaxies: evolution — cosmology: theory

\section{INTRODUCTION}

A wide range of observed phenomena support the view that gas-rich mergers play a central role in galaxy evolution. The most intense starbursts, ultraluminous infrared galaxies (ULIRGs), are always associated with mergers (e.g. Joseph \& Wright 1985; Sanders \& Mirabel 1996), with dense gas in their centers providing material to feed black hole $(\mathrm{BH})$ growth and to boost the concentration and central phase space density of merging spirals to match those of ellipticals (Hernquist et al. 1993; Robertson et al. 2006b). Studies of ongoing mergers and remnants from mergers of gas-rich systems (e.g., Lake \& Dressler 1986; Doyon et al. 1994; Shier \& Fischer 1998; James et al. 1999; Genzel et al. 2001; Tacconi et al. 2002; Dasyra et al. 2006, 2007; Rothberg \& Joseph 2004, 2006a), as well as poststarburst $(\mathrm{E}+\mathrm{A} / \mathrm{K}+\mathrm{A})$ galaxies (Goto 2005), have shown that the kinematic and photometric properties of these objects, including velocity dispersions, concentrations, stellar masses, light profiles, and phase space densities, are consistent with their eventual evolution into typical $\sim L_{*}$ elliptical galaxies.

The correlations obeyed by these mergers and remnants (e.g., Genzel et al. 2001; Tacconi et al. 2002; Rothberg \& Joseph 2006a b; Dasyra et al. 2007) are similar to e.g. the observed fundamental plane and Kormendy relations for relaxed ellipticals, and indicate that they will evolve onto these relations as their stellar populations age.

\footnotetext{
${ }^{1}$ Harvard-Smithsonian Center for Astrophysics, 60 Garden Street, Cambridge, MA 02138, USA

${ }^{2}$ W. M. Keck Postdoctoral Fellow at the Harvard-Smithsonian Center for Astrophysics

${ }^{3}$ Space Telescope Science Institute, 3700 San Martin Drive, Baltimore, MD 21218, USA

${ }^{4}$ Naval Research Laboratory, Remote Sensing Division, Code 7211, Washington, DC 20375, USA
}

This is further supported by the ubiquitous presence of fine structures such as shells, ripples, and tidal plumes in ordinary ellipticals (e.g. Schweizer \& Seitzer 1992; Schweizer 1996), which are signatures of mergers (e.g. Ouinn 1984; Hernquist \& Ouinn 1987; Hernquist \& Spergel 1992), and the clustering and mass density of ellipticals, which are consistent with passive evolution after formation in mergers (Hopkins et al. 2007c). These various lines of evidence are in accord with the "merger hypothesis" (Toomre \& Toomre 1972; Toomre 1977), that elliptical galaxies originate from mergers of spirals.

Numerical simulations performed during the past twenty years verify that major mergers of gas-rich disk galaxies can plausibly account for these phenomena and elucidate the underlying physics. In Hopkins et al. (2008d), we describe the phases of evolution characterizing these mergers and their relationship to quasars, starbursts, and elliptical galaxies; we briefly summarize these phases here. Tidal torques excited during a merger drive rapid inflows of gas into the centers of galaxies (Barnes \& Hernquist 1991, 1996), triggering starbursts (Mihos \& Hernquist 1996; Springel \& Hernquist 2003) and feeding rapid black hole growth (Di Matteo et al.|2005). Gas consumption by the starburst and dispersal of residual gas by supernova-driven winds and feedback from black hole growth (Springel et al. 2005a) terminate star formation so that the remnant quickly evolves from a blue to a red galaxy. The stellar component of the progenitors provides the bulk of the material for producing the remnant spheroid (Barnes 1988, 1992; Hernquist 1992, 1993b) through violent relaxation, but the highest phase-space density material and central "cusp" come from dissipation in the final, merger-induced starburst.

A major merger is generally required in order for the tidal forces to excite a sufficiently strong response to set up nuclear inflows of gas and build realistic spheroids. Although 
simulations suggest that the precise definition of a major merger in this context is somewhat blurred by the degeneracy between the mass ratio of the progenitors and the orbit of the interaction (Hernquist 1989; Hernquist \& Mihos 1995; Bournaud et al. 2005), numerical studies (Younger et al. 2007a.b) and observations (Dasyra et al. 2006; Woods et al. 2006) find that strong gas inflows and morphological transformation are typically observed only for mass ratios below $\sim 3: 1$, despite the greater frequency of higher mass-ratio mergers. In what follows, unless explicitly noted, we use the term "mergers" to refer specifically to major mergers (mass ratio $\lesssim 3: 1$ ).

Gas dissipation and star formation in the compact, mergerinduced central starburst are key ingredients in these simulations and observations. It has long been recognized that many properties of ellipticals cannot be reproduced by purely dissipationless mergers of late-type galaxies (Ostriker 1980; Carlberg 1986; Naab \& Ostriker 2007): in particular, their high central phase-space densities and specific frequency of global clusters (e.g. Schweizer 1998), degree of rotational support, anisotropy, and minor axis rotation/kinematic misalignment (Barnes \& Hernquist 1996; Cox et al. 2006b; Naab et al. 2006), and isophotal shapes. However, these arguments do not apply when the merger constituents contain gas, which can radiate energy and hence increase phase-space densities (Lake 1989) and form new stellar populations.

Observed absorption-line spectra and the red colors of elliptical galaxies suggest that their stars formed at high redshift $(z \gtrsim 1)$ and that little star formation has since occurred in them. If these galaxies were produced by mergers, the progenitors of many present-day ellipticals were high-redshift spirals. While relatively little is known about disk galaxies at high redshift, it is likely that they were more concentrated and more gas-rich than their low-redshift counterparts. Indeed, observational evidence (Erb et al. 2006) indicates that galaxies at redshift $z \sim 2$ do have large gas fractions $f_{\text {gas }} \sim 0.5$, with some approaching $f_{\text {gas }} \sim 0.8-0.9$. Thus, any attempt to understand the formation, properties, and scaling relations of the present-day population of elliptical galaxies within the context of the merger hypothesis must consider gas-rich mergers.

In pioneering studies employing simulations that incorporated gas-dynamics and star formation, Mihos \& Hernquist (1994a c) demonstrated that dissipation in disk-disk mergers leads to central starbursts similar to those observed in LIRGs and ULIRGs, leaving behind stellar populations that easily match the central phase space and surface densities of observed ellipticals. In particular, Mihos \& Hernquist (1994a) (hereafter, MH94) predicted that this process should imprint an observable signature in the surface brightness profiles of ellipticals, in the form of a steep departure from the outer de Vaucouleurs (1948) $\sim r^{1 / 4}$-law (which arises from violent relaxation of stars in the pre-merger disks) in the inner regions: i.e. the presence of a central cusp or extra light above the inwards extrapolation of the outer profile. Subsequent observations (Hibbard \& Yun 1999; Rothberg \& Joseph 2004) have now confirmed the existence of such excess light in at least a significant fraction of known gas-rich merger remnants. In principle, this could provide an indicator of how much mass was involved in the dissipational starburst, and therefore a critical constraint on the formation history of the galaxy. Hibbard \& Yun (1999); Rothberg \& Joseph (2004) also made first attempts to quantify the fraction of stellar mass or light involved; however, without a detailed compari- son with theoretical models to provide the appropriate "null" model, it is not clear how exactly to quantify the amount of "excess" in the light profiles.

An estimate of how much gas is required to match the central densities of ellipticals was provided by Hernquist et al. (1993), who used N-body simulations and analytic arguments to suggest that, without such a dissipational component, mergers would be deficient by $\sim 15 \%$ of their mass relative to the inward extrapolation of an $r^{1 / 4}$ law. Naab \& Trujillo (2006) found a similar deficit by comparing a sample of surface density profiles from collisionless (gas-free) disk merger remnants and observed merger remnants (but with reasonable agreement in profile shapes in the outer regions). Since true $\lesssim L_{*}$ ellipticals have a variety of profiles reflected in their Sersic indices, $I \propto \exp \left\{-\left(r / r_{0}\right)^{1 / n}\right\}$, with $n \sim 2-4$, this implies a range of extra light fractions $\sim 1-20 \%$ needed to account for realistic populations of $\lesssim L_{*}$ ellipticals.

At the time of the predictions made by MH94, these central cusps or extra light components, the relics of dissipational formation in a gas-rich merger, had not been observed. However, with the advent of Hubble Space Telescope observations of the centers of elliptical galaxies, it is now well-established that typical $\lesssim L_{*}$ ellipticals exhibit such central light excesses, with mass ranges and radii comparable to those originally predicted by MH94 (Kormendy 1999; Kormendy et al. 2007; Ferrarese et al.2006; Cote et al.2007). The situation is somewhat different for the most massive ellipticals, which appear to have central "cores" or "missing light," generally accompanied by slow rotation and boxy isophotal shapes (Faber et al. 1997). It is believed, however, that this family of ellipticals has been further modified by subsequent re-mergers or "dry mergers" of two or more (initially cuspy gas-rich merger remnant) ellipticals (Quillen et al. 2000; Rest et al. 2001; Ravindranath et al. 2001; Laine et al. 2003; Lauer et al. 2005, 2007; Ferrarese et al. 2006; Cote et al. 2007; Kormendy et al. 2007). While the central profiles of these objects are certainly interesting (and are considered in Hopkins et al. (2008a)), they do not directly inform the history of gas-rich mergers and are therefore not the subject of our investigation here.

In principle, there is great potential for understanding galaxy formation histories and structural properties by identifying observed cusps with those predicted as the relics of gas-rich central starbursts by MH94. These cusps could enable observers to determine individually the masses involved in violent relaxation versus dissipation in the merger, and to separate the stellar populations arising from both processes, further informing the origin of radial gradients in stellar age, metallicity, and abundance in ellipticals (e.g. Mihos \& Hernquist 1994b). The importance and fractional contribution of dissipation as a function of elliptical properties such as mass and formation time could be assessed, and this could further be used as a direct test for the role of differential dissipation being a driver in e.g. the tilt of the fundamental plane, as suggested by theoretical models (Robertson et al. 2006b; Dekel \& Cox 2006). Correlations with global kinematic properties can constrain and test theories, like those outlined above, that invoke dissipation to resolve long-standing objections to the merger hypothesis. The proposed links between ULIRGs, quasars, and elliptical galaxy formation in mergers (e.g. Sanders \& Mirabel 1996; Hopkins et al. 2006a b, 2008c d) similarly rely on dissipation occurring in mergers and, hence, anticipate that it should have a profound impact on the centers of ellipticals. 
Unfortunately, despite the major improvements in both observations of the centers of elliptical galaxies and numerical treatments of star formation and gas physics, there has been little attempt to use the extra light content of ellipticals as probes in the manner above. Rather, studies have mainly been limited to determining whether or not some extra light component is evident. This owes in large part to a lack of theoretical underpinning: the original MH94 work simply predicted that such cusps should exist; more detailed interpretation and modeling was limited by spatial and numerical resolution, and the inability to characterize gas physics with realistic empirically calibrated models for star formation and supernova feedback in the interstellar medium. There have been dramatic improvements in these areas in recent years (e.g. Springel \& Hernquist 2003; Springel et al. 2005b), but the issue has not been revisited.

Here, and in a companion paper (Hopkins et al. 2008b), we examine galaxy cusps or extra light components in both simulations and observed galaxies, in order to identify the origin of different components of elliptical surface density profiles, their cosmological scalings and relevance for the formation history of such galaxies, and their implications for global galaxy properties. In the present paper, we focus on the extra light in our simulations and in known gas-rich merger remnants, in order to isolate the relevant physics and to calibrate a means to recover the most important physical quantities involved.

In $\S 2$ we describe our suite of gas-rich merger simulations, and in $\S 3$ we summarize the observational data set used in our comparisons. In $\S 4$ we highlight the physical origin and nature of cusp or extra light components. In $\S 5$ we compare different means by which to fit the surface density profile, and attempt to calibrate fitting methods in order to recover the physically distinct (dissipational versus dissipationless) components in merger remnants. In $\S[$ we briefly discuss the effects of resolution and similar numerical issues involved, before proceeding in $\S 7$ to compare our simulations and fitted galaxy decomposition to observed systems. In $\$ 8$ we investigate how the amount of extra light depends on the gas content of the merging galaxies. In $\S 9$ we use these comparisons to study how structural parameters of the outer stellar light and inner extra light component scale with e.g. galaxy mass and size. In $\$ 10$ we calculate how these results and comparisons are affected by the choices of observed wavebands and time evolution after the merger. Finally, in $\S 11$ we discuss our results and outline future explorations of these correlations.

Throughout, we adopt a $\Omega_{\mathrm{M}}=0.3, \Omega_{\Lambda}=0.7, H_{0}=$ $70 \mathrm{~km} \mathrm{~s}^{-1} \mathrm{Mpc}^{-1}$ cosmology, and normalize all observations and models shown to this cosmology. We also adopt a Chabrier (2003) initial mass function (IMF), and convert all stellar masses and mass-to-light ratios to this choice. The exact choice of IMF systematically shifts the normalization of stellar masses herein, but does not substantially change our comparisons. All magnitudes are in the Vega system, unless otherwise specified.

\section{THE SIMULATIONS}

Our simulations were performed with the parallel TreeSPH code GADGET-2 (Springel 2005), employing the fully conservative formulation (Springel \& Hernquist 2002) of smoothed particle hydrodynamics (SPH), which conserves energy and entropy simultaneously even when smoothing lengths evolve adaptively (see e.g., Hernquist 1993a; O'Shea et al. 2005). Our simulations account for radiative cooling, heating by a
UV background (as in Katz et al. 1996; Davé et al. 1999), and incorporate a sub-resolution model of a multiphase interstellar medium (ISM) to describe star formation and supernova feedback (Springel \& Hernquist 2003). Feedback from supernovae is captured in this sub-resolution model through an effective equation of state for star-forming gas, enabling us to stably evolve disks with arbitrary gas fractions (see, e.g. Springel et al. 2005b; Springel \& Hernquist 2005; Robertson et al. 2006a.c). This is described by the parameter $q_{\text {eos }}$, which ranges from $q_{\text {eos }}=0$ for an isothermal gas with effective temperature of $10^{4} \mathrm{~K}$, to $q_{\text {eos }}=1$ for our full multiphase model with an effective temperature $\sim 10^{5} \mathrm{~K}$. We also compare with a subset of simulations which adopt the star formation feedback prescription from Mihos \& Hernquist (1994a b c, 1996), in which the ISM is treated as a singlephase isothermal medium and feedback energy is deposited as a kinetic impulse.

Although they make little difference to the extra light component, supermassive black holes are usually included at the centers of both progenitor galaxies. These black holes are represented by "sink" particles that accrete gas at a rate $\dot{M}$ estimated from the local gas density and sound speed using an Eddington-limited prescription based on Bondi-HoyleLyttleton accretion theory. The bolometric luminosity of the black hole is taken to be $L_{\mathrm{bol}}=\epsilon_{r} \dot{M} c^{2}$, where $\epsilon_{r}=0.1$ is the radiative efficiency. We assume that a small fraction (typically $\approx 5 \%$ ) of $L_{\text {bol }}$ couples dynamically to the surrounding gas, and that this feedback is injected into the gas as thermal energy, weighted by the SPH smoothing kernel. This fraction is a free parameter, which we determine as in Di Matteo et al. (2005) by matching the observed $M_{\mathrm{BH}}-\sigma$ relation. For now, we do not resolve the small-scale dynamics of the gas in the immediate vicinity of the black hole, but assume that the timeaveraged accretion rate can be estimated from the gas properties on the scale of our spatial resolution (roughly $\approx 20 \mathrm{pc}$, in the best cases).

The progenitor galaxy models are described in Springel et al. (2005b), and we review their properties here. For each simulation, we generate two stable, isolated disk galaxies, each with an extended dark matter halo with a Hernquist (1990) profile, motivated by cosmological simulations (Navarro et al. 1996; Busha et al. 2005), an exponential disk of gas and stars, and (optionally) a bulge. The galaxies have total masses $M_{\mathrm{vir}}=V_{\mathrm{vir}}^{3} /\left(10 G H_{0}\right)$ for $z=0$, with the baryonic disk having a mass fraction $m_{\mathrm{d}}=0.041$, the bulge (when present) having $m_{\mathrm{b}}=0.0136$, and the rest of the mass in dark matter. The dark matter halos are assigned a concentration parameter scaled as in Robertson et al. (2006c) appropriately for the galaxy mass and redshift following Bullock et al. (2001). We have also varied the concentration in a subset of simulations, and find it has little effect on our conclusions (because the central regions of the galaxy are, in any case, baryon-dominated). The disk scale-length is computed based on an assumed spin parameter $\lambda=0.033$, chosen to be near the mode in the $\lambda$ distribution measured in simulations (Vitvitska et al. 2002), and the scale-length of the bulge is set to 0.2 times this.

Typically, each galaxy initially consists of 168000 dark matter halo particles, 8000 bulge particles (when present), 40000 gas and 40000 stellar disk particles, and one BH particle. We vary the numerical resolution, with many simulations using twice, and a subset up to 128 times, as many particles. We choose the initial seed mass of the black hole either in 
accord with the observed $M_{\mathrm{BH}^{-}} \sigma$ relation or to be sufficiently small that its presence will not have an immediate dynamical effect, but we have varied the seed mass to identify any systematic dependencies. Given the particle numbers employed, the dark matter, gas, and star particles are all of roughly equal mass, and central cusps in the dark matter and bulge are reasonably well resolved.

We consider a series of several hundred simulations of colliding galaxies, described in detail in Robertson et al. (2006b c) and Cox et al. (2006a b). We vary the numerical resolution, the orbit of the encounter (disk inclinations, pericenter separation), the masses and structural properties of the merging galaxies, presence or absence of bulges in the progenitor galaxies, initial gas fractions, halo concentrations, the parameters describing star formation and feedback from supernovae and black hole growth, and initial black hole masses.

The progenitor galaxies have virial velocities $V_{\text {vir }}=$ $55,80,113,160,226,320$, and $500 \mathrm{~km} \mathrm{~s}^{-1}$, and redshifts $z=$ $0,2,3$, and 6 , and our simulations span a range in final spheroid mass $M_{\mathrm{BH}} \sim 10^{8}-10^{13} M_{\odot}$, covering essentially the entire range of the observations we consider at all redshifts, and allowing us to identify any systematic dependencies in our models. We consider initial disk gas fractions (by mass) of $f_{\text {gas }}=0.05,0.1,0.2,0.4,0.6,0.8$, and 1.0 for several choices of virial velocities, redshifts, and ISM equations of state. The results described in this paper are based primarily on simulations of equal-mass mergers; however, by examining a small set of simulations of unequal mass mergers, we find that the behavior does not change dramatically for mass ratios below about $3: 1$ or $4: 1$. This range is appropriate to the observations of merging galaxies used in this paper, which are restricted to major merger events. At higher mass ratios, our experiments (Younger et al. 2007a) suggest that gas can be channeled by a minor merger into the central regions of the galaxy, with an efficiency that declines in approximately linear fashion with the mass ratio. However, the resulting starburst forms a small bulge in a disk galaxy, so the decomposition into a Sersic profile representing the scattered stars and extra light component becomes ambiguous (both physically and observationally).

Each simulation is evolved until the merger is complete and the remnants are fully relaxed, typically $\sim 1-2$ Gyr after the final merger and coalescence of the BHs. We then analyze the remnants following Cox et al. (2006b), in a manner designed to mirror the methods typically used by observers. For each remnant we project the stars onto a plane as if observed from a particular direction (we consider 100 viewing angles to each remnant, which uniformly sample the unit sphere). Given the projected stellar mass distribution, we calculate the iso-density contours and fit ellipses to each (fitting major and minor axis radii and hence ellipticity at each iso-density contour), moving concentrically from $r=0$ until the entire stellar mass has been enclosed. This is designed to mimic observational isophotal fitting algorithms (e.g. Bender et al. 1987; Bender 1988). The radial deviations of the iso-density contours from the fitted ellipses are expanded in a Fourier series in the standard fashion to determine the boxiness or diskiness of each contour (the $a_{4}$ parameter). Throughout, we show profiles and quote our results in terms of the major axis radius. For further details, we refer to Cox et al. (2006b).

We directly extract the effective radius $R_{\mathrm{e}}$ as the projected stellar half-mass radius, and the velocity dispersion $\sigma$ as the average one-dimensional velocity dispersion within a circular aperture of radius $R_{\mathrm{e}}$. Note that this differs from what is sometimes adopted in the literature, where $R_{\mathrm{e}}$ is determined from the best-fit Sersic profile, but because we are fitting Sersic profiles to the observed systems we usually quote both the true effective radius of the galaxy and effective radii of the fitted Sersic components. Throughout, the stellar mass $M_{*}$ refers to the total stellar mass of the galaxy, and the dynamical mass $M_{\text {dyn }}$ refers to the traditional dynamical mass estimator

$$
M_{\text {dyn }} \equiv k \frac{\sigma^{2} R_{\mathrm{e}}}{G},
$$

where we adopt $k=4.2$ (roughly what is expected for a Hernquist (1990) profile, and the choice that most accurately matches the true enclosed stellar plus dark matter mass within $R_{\mathrm{e}}$ in our simulations; although this choice is irrelevant as long as we apply it uniformly to both observations and simulations). When we plot quantities such as $R_{\mathrm{e}}, \sigma$, and $M_{\text {dyn }}$, we typically show just the median value for each simulation across all $\sim 100$ viewing directions. The sightline-tosightline variation in these quantities is typically smaller than the simulation-to-simulation scatter, but we explicitly note where it is large.

\section{THE OBSERVATIONS}

We compare these simulations with the sample of local remnants of recent gas-rich mergers from Rothberg \& Joseph (2004) (henceforth RJ04). For these objects, RJ04 compile $K$-band imaging, surface brightness, ellipticity, and $a_{4} / a$ profiles, where the profiles typically range from $\sim 100 \mathrm{pc}$ to $\sim 10-20 \mathrm{kpc}$. These span a moderate range in luminosity (including objects from $M_{K} \sim-20$ to $M_{K} \sim-27$, but with most from $M_{K} \sim-24$ to $M_{K} \sim-26$ ) and a wide range in merger stage, from ULIRGs and (a few) unrelaxed systems to shell ellipticals. As demonstrated therein, these systems will generally become (or already are, depending on the classification scheme used) typical $\sim L_{*}$ ellipticals, with appropriate phase space densities, surface brightness profiles, fundamental plane relations, kinematics, and other properties.

In terms of direct comparison with our simulations, the data often cover a dynamic range and have resolution comparable to our simulations, and (experimenting with different smoothings and imposed dynamic range limits) we find it is unlikely that resolution or seeing differences will substantially bias our comparisons. We have converted the observations to our adopted cosmology, and compile other global parameters including e.g. kinematic properties and luminosities in other bands from Rothberg \& Joseph (2006a, b) and the literature. We estimate stellar masses ourselves in a uniform manner for all the objects given the mean $K$-band $M / L$ as a function of luminosity from Bell et al. (2003), corrected for our adopted IMF. For objects where optical photometry is available, we have repeated our analysis using stellar masses derived from total $K$-band luminosities and $(B-V)$ color-dependent massto-light ratios from Bell et al. (2003) or from fitting integrated $U B V R I J H K$ photometry of each object to a single stellar population with the models of Bruzual \& Charlot (2003), and find this makes no difference to our conclusions. Of course, since these are young objects and we are not performing full SED fitting, there is some uncertainty in these estimates. We find a similar result, however, using dynamical masses from the kinematics in Rothberg \& Joseph (2006a) - although these too are uncertain possibly owing to the incomplete relaxation of the systems. Within the considerable scatter in the observed properties, however, we find these details makes little difference. 
Throughout, we will usually refer interchangeably to the observed surface brightness profiles in the given bands and the surface stellar mass density profile. Of course, stellar light is not exactly the same as stellar mass, but in $\S 10$ we consider the differences between the stellar light and the stellar mass density profiles as a function of time, wavelength, and properties of the merger remnant, and show that the $K$-band results introduce little bias (i.e. are good tracers of the stellar mass); the Sersic indices and extra light fractions fitted to the $K$-band profiles of the simulations are good proxies for the Sersic index of the stellar mass profile and extra mass/starburst mass fraction, even close in time to the peak episode of star formation. As has been noted in other works, many of these systems have weak color gradients, empirically supporting the idea that there is generally only weak variation in $M / L$ with radius.

It is important to note that while we are not concerned about the absolute normalization of the profile (i.e. mean $M / L$ ), since we derive total stellar masses separately from the integrated photometry, we must account for systematics that might be induced by change in $M / L$ as a function of radius. The profiles in optical bands such as $V$ require more care when the system is very young $(\lesssim 1-2 \mathrm{Gyr}$ after the major merger-induced peak of star formation), there can be considerable bias or uncertainty owing to stellar population gradients and dust. However, once the system is relaxed, the optical bands also become good proxies for the stellar mass distribution.

\section{THE PHYSICAL ORIGIN OF "EXTRA LIGHT"}

We begin by considering a fiducial case which illustrates many of the physical processes relevant to the origin of extra light in gas-rich galaxy mergers. Figure 1 shows the surface mass density of stars (from a random sightline) of a typical, but extremely gas-rich, simulation. The merging galaxies are similar to $\sim L_{*}$ disk galaxies (Sc-type galaxies) with a large initial gas fraction $f_{\text {gas }} \sim 0.8$, and merge on an orbit representative of most random encounters. Despite the large gas content, the total stellar mass profile is quite smooth, at least down to the resolution limit of this particular simulation $(\sim 50 \mathrm{pc})$. However, there is a suggestion of a central extra light concentration or cusp within $\sim 0.5-1 \mathrm{kpc}$. (We choose a high gas fraction case merely to illustrate the salient features of our findings; as we detail in what follows, mergers with lower gas fractions yield qualitatively similar results.)

The simulation allows us to decompose the stellar mass profile according to the time when various stellar populations were formed. We therefore consider three classes of stars. First, the stars that were present in the initial stellar disks at the beginning of the simulation ("disk stars") together with those stars formed before the final coalescence of the galaxies and starburst phase ("pre-starburst" stars). Although the latter includes some stars that originate in a small starburst caused by the first passage of the two galaxies, most of these stars are formed quiescently in the two galactic disks before the disks are destroyed in the final merger. We identify these two simulated classes of stars as a single population because they are both "disk" stars entering the final merger, and it is of course arbitrary where (or at what time) we initialize our simulations, relative to the final merger, and therefore what fraction of the stellar mass forms "before" our simulation begins. As is clear in the figure, these stars are scattered during the merger and undergo violent relaxation, producing an $r^{1 / 4}$-like surface density profile. In detail, these stars relax to a nearly perfect Sersic profile with a best-fit Sersic index of $n_{s} \approx 4.0$, which is shown in the right panel of Figure 1 As stars, they are collisionless, and therefore remain at large radii and relatively low phase-space densities characteristic of the scale lengths and concentrations of the initial disks, with an effective radius $R_{\mathrm{e}} \approx 5 \mathrm{kpc}$.

Second is the "starburst" component, defined somewhat loosely as those stars which form within $250 \mathrm{Myr}$ of the final merger and coalescence of the two nuclei (taken for convenience as the merger of the two central black holes, but adopting another definition such as the peak in the star formation rate yields a similar result). This component is much more compact $\left(R_{\mathrm{e}}=0.2 \mathrm{kpc}\right)$, characteristic of the central starburst size scales in observed mergers and ULIRGs (e.g. Downes \& Solomon 1998; Bryant \& Scoville 1999; Genzel et al. 2001; Tacconi et al. 2002). Because our definition is based on only the time during which the stars formed, it includes some stars which originate in the merging disks (rather than the central, compact starbursts), which can be seen in the tails of this component of the surface density profile. However, these contribute negligibly to the surface brightness profile and total mass budget of the starburst component. The central component does not follow an $r^{1 / 4}$ law, but is more similar to an exponential $\left(n_{s}=1\right)$ profile. We will return to the details of the exact shape of this central light component in $\S 9$, but in general expect this - the central starburst is formed in situ by gas dissipation and star formation, and does not undergo violent relaxation to transform it from an exponential-like to an $r^{1 / 4}$ profile.

Finally, because of the extremely gas-rich nature of this merger, there is some amount of cold gas remaining after the final black hole growth and feedback phase. This quickly settles into a rotationally supported disk, and forms an embedded disk of "post-starburst" stars (described in detail in Springel \& Hernquist 2005). Figure 1 shows that this can be well-fitted by a relatively small exponential disk $\left(R_{\mathrm{e}} \sim 3 \mathrm{kpc}\right)$. Although this embedded disk is interesting from the perspective of the galaxy kinematics (see Cox et al. 2006b), it contains only a tiny fraction $\sim 4 \%$ of the stellar mass, and, because this is concentrated near the total galaxy effective radius, it adds negligibly to the surface brightness profile at all radii.

We therefore confirm the findings of MH94, that gas channeled to the central regions of the galaxy forms stars in a compact starburst and leaves a central excess in the light profile above the extrapolation of the light of the old stellar population (scattered from the disks of the merging galaxies). However, even in Figure 1, where the extra light or starburst component constitutes a large fraction $(\sim 30 \%)$ of the galaxy stellar mass, the overall light profile is quite smooth, and does not show an obvious sharp transition to the extra light component. This is unlike the earlier results of MH94, and we discuss some of the reasons for this in $\S 9$, but they primarily relate to our improved resolution and treatment of the ISM relative to $\mathrm{MH} 94$, as well as more accurate time integration.

\section{FITTING TO RECOVER THE PHYSICALLY APPROPRIATE "EXTRA LIGHT"}

We would like to be able to use the surface brightness profile of the merger remnant to estimate the contribution of the extra light - ideally as a means of estimating, from the amount of extra light, how much of the galaxy mass was involved in a compact central starburst. However, as noted in $\$ 4$, the light profiles in our simulation remnants are quite smooth, even 


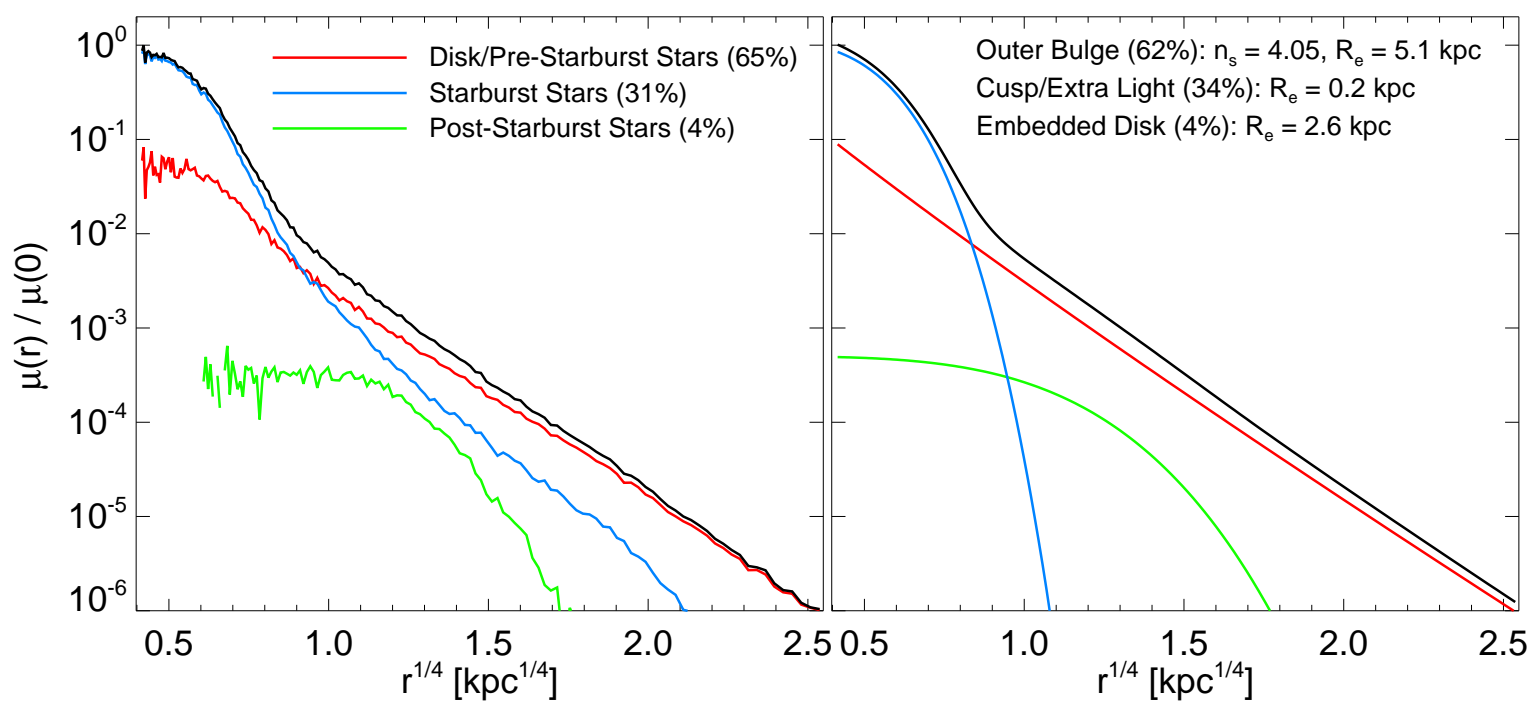

FIG. 1.- Projected surface mass density of stars in the remnant of a highly gas-rich merger. Left: Total profile (black) is shown with contribution from various physical components: stars in pre-simulation disks and those formed in the simulated disks before the final merger (disk/pre-starburst stars), stars formed in the compact, merger-induced starburst at final coalescence (starburst stars), and stars formed from gas which survives the merger (post-starburst stars). Right: Sersic profile fits to each of these components, labeled as "outer bulge," "cusp/extra light," and "embedded disk," respectively (sum shown in black). Mass fractions, Sersic indices $\left(n_{s}\right)$ and effective radii of each component are plotted ( $n_{s}=1$ for the embedded disk and cusp/extra light component).

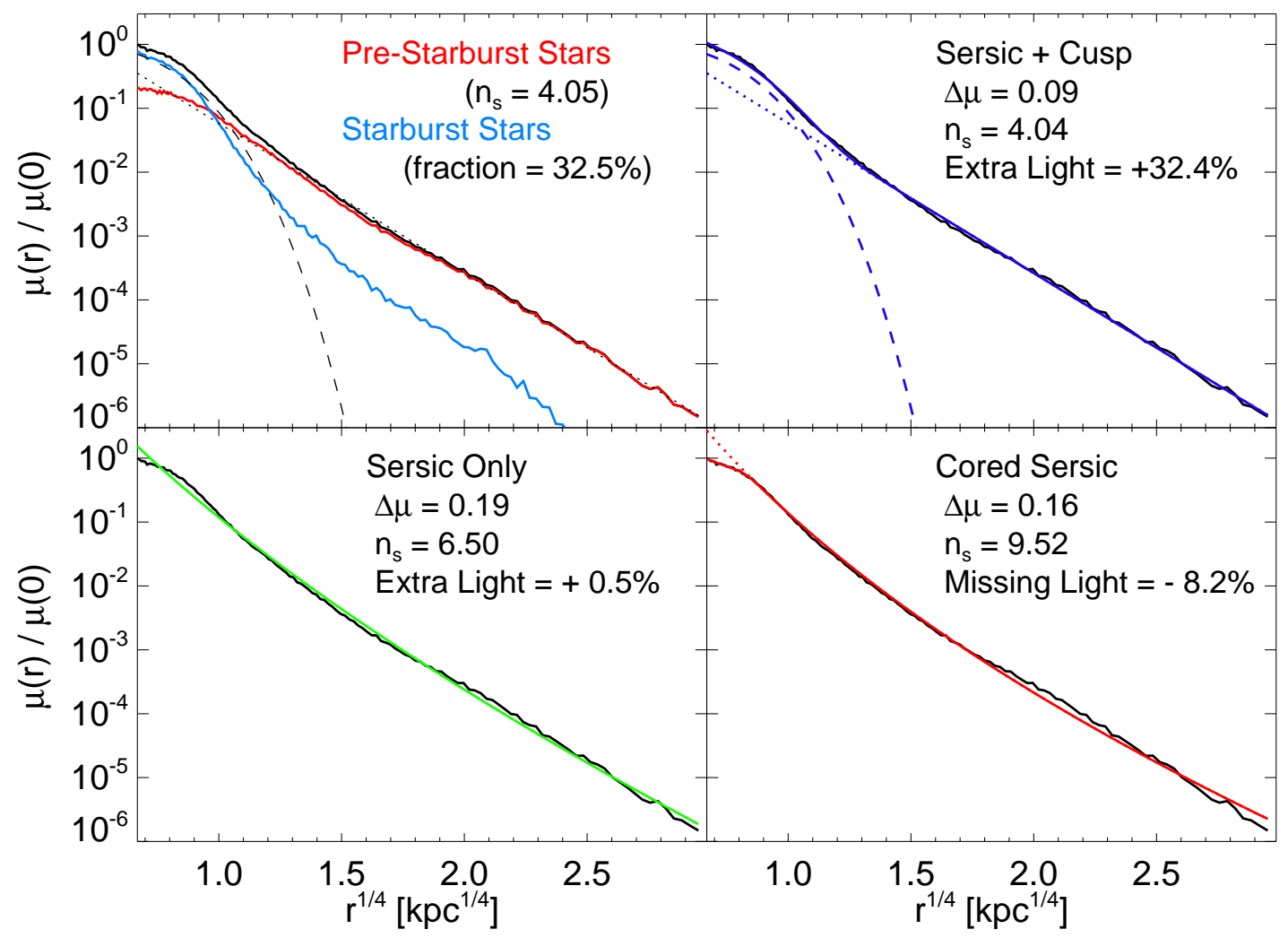

FIG. 2.- Upper Left: Surface mass density profile of remnant of a highly gas-rich merger (black), decomposed into stars formed prior to the final merger (which are then violently relaxed; red) and stars formed in the merger-driven dissipational starburst (blue). The Sersic fit to each component is shown, with the Sersic index of the pre-starburst component and mass fraction of the starburst component labeled. Upper Right: Two-component (Sersic plus cusp or extra light; where the cusp is fit with an exponential profile) fit to the total light profile, with the Sersic index of the outer component and mass fraction of the inner $\left(n_{s}=1\right)$ component, and rms scatter $(\Delta \mu)$ about the fit. Lower Left: Single Sersic function fit to the profile. Lower Right: Cored Sersic function fit. The two-component fit as we parameterize it accurately recovers the Sersic profile of the violently relaxed component and mass fraction of the starburst component. The other fits give physically misleading results in this case. 
where the extra light fraction is large. This makes identifying the extra light component a non-trivial procedure, which is sensitive to the assumptions made in fitting.

We begin by reducing a galaxy surface density profile to the two most relevant physical components: the "pre-starburst" or "disk" stars, i.e. those formed in the rotationally supported disks before the final coalescence of the galaxies, and the "starburst" stars, formed in the compact, dissipational final starburst. We neglect the embedded stellar disks which can be formed by gas remaining after the merger; as demonstrated in Figure 1, these contribute negligibly to the surface mass density profile in even the most gas-rich merger remnants.

Figure 2 shows the surface density of a fiducial gas-rich example, decomposed into a pre-starburst and starburst population. Motivated by observational methods and our results from $\S$ 4, we $\mathrm{fit}^{5}$ (upper left) a Sersic profile to the prestarburst component, and find that it follows a nearly perfect $r^{1 / 4}$ law $\left(n_{s}=4.05\right)$. Loosely motivated by the shape of the starburst stellar profile at small radii and its origin in a gas-rich dissipational starburst, we fit the starburst stellar component to an exponential Sersic law $\left(n_{s}=1\right)$. We emphasize that neither the simulations nor observations herein resolve the innermost regions of the central light where the choice of its Sersic index makes a significant difference, so we adopt this for simplicity as a standard choice in fitting the extra light component (we discuss this choice in greater detail below and $\$ 9$ ). This describes the starburst mass profile quite well where it is important to the overall surface density. It does not capture the extended stars at $\gtrsim 3 \mathrm{kpc}$ formed during this time, but as discussed in $\$ 4$, we can safely ignore them. The exponential fit accurately recovers the total mass in the starburst component and its effective radius, and the shape of this profile where it is relevant to the total surface brightness profile.

Rather than fit directly to the physical starburst and prestarburst components, we now attempt to fit a two-component model to the observed quantity, the total surface brightness profile (upper right). We therefore fit the surface brightness profile to a double Sersic model, with an outer component reflecting the pre-starburst stars with a free Sersic index, and an inner component reflecting the starburst stars with a fixed Sersic index $n_{s}=1$. The total surface brightness profile is then

$$
I_{\mathrm{tot}}=I_{s b} \exp \left\{-b_{1}\left(\frac{r}{r_{s b}}\right)\right\}+I_{b} \exp \left\{-b_{n}\left(\frac{r}{r_{b}}\right)^{1 / n}\right\},
$$

where $r_{s b}$ and $r_{b}$ are the effective radii of the $n_{s}=1$ and free $n_{s}$ components (which we identify with the "starburst" and "old bulge" or "pre-starburst" components, respectively), $I_{s b}$ and $I_{b}$ are the corresponding normalizations, and $n$ is the Sersic index of the outer bulge or pre-starburst component. The constant $b_{n}$ is the appropriate function of $n$ such that $r_{s b}$ and $r_{b}$ correspond to the projected half-mass radii. Note that changing our choice of $n_{s}=1$ for the inner Sersic component will systematically shift the mass balance between the inner and outer components, and lower the fitted outer Sersic index (typically increasing the inner mass by $\sim 30 \%$ and lowering the outer Sersic index $\Delta n_{s} \sim 0.25$, respectively, if we adopt

\footnotetext{
5 Formally, we consider our simulation profiles outside of some multiple $\sim 3-5$ times the resolution limit, or with a seeing correction appropriate for the RJ04 sample (much larger than our resolution limits). We equally sample the profile in $\log r$ over a dynamic range extending to the largest radii in the RJ04 sample, and weight each point equally assuming an intrinsic $\sim 0.1 \mathrm{mag}$ point-to-point variance in the SB profile (the typical magnitude of residuals fitting arbitrary splines to the data). We have varied these choices and find that our fits and conclusions are not sensitive to them.
}

$\left.n_{s}=2\right)$.

We have therefore experimented with a range of choices for the inner Sersic index: we find that fixing $n_{s}=1$ recovers, on average (across our entire suite of simulations), the correct mass of the physical starburst component and Sersic index of the pre-starburst stellar component, whereas different choices for the inner $n_{s}$ give systematic offsets in these quantities. This is not to say that $n_{s}=1$ is most appropriate for every object/sightline or that it yields the most physical results in every case: however, if we are interested in recovering the physical extra light component in a statistical sense, it is most appropriate. Furthermore, even where we arrive at a similar result if we fit it with a free Sersic index, we find that this introduces considerably larger uncertainties when we fit observed systems (owing to the various parameter degeneracies).

Figure 2 shows the outcome of this fit. The resulting model of the surface density profile fits the simulation well, with a variance of only $\Delta \mu \sim 0.09$ mag $\operatorname{arcsec}^{-2}$ (assuming $\left.\mu \propto-2.5 \log I_{\text {tot }}\right)$. This is comparable to the point-to-point variance in the profile of this simulation if we fit an arbitrary spline to the data, and thus reflects a genuinely good fit. We have tested and found that the fit parameters are stable with respect to the dynamic range and error weighting, and that the residuals (typically of this magnitude, $\Delta \mu \sim 0.1$ mag arcsec ${ }^{-2}$ ) are independent of radius (i.e. there is no systematic bias in the functional form). More important, this fit, despite having no direct information about the physical components into which we decompose the brightness profile, recovers almost exactly the appropriate parameters for both profiles. The bestfit Sersic index $\left(n_{s}=4.04\right.$ compared to 4.05$)$ and effective radius of the "outer" or "bulge" component are almost perfect matches to those fitted directly to the pre-starburst stellar population. Likewise, the inner or extra light component is a close match to the physical starburst component, and the fit recovers the extra light fraction accurately (32.4\% compared to $32.5 \%$; a much smaller difference than reasonable uncertainties in our physical definition of the starburst component). We have repeated this fitting procedure for several hundred simulations, and find in almost all cases similar good agreement between the best-fit components and the physical decomposition of the galaxy stellar mass profile.

However, there are a number of local minima in the fitting, and care must be taken to fit the most appropriate physical model. We have, in some sense, tuned our two-component parameterization (choosing the functional form and fixing the inner $n_{s}=1$ ) so that it, on average, accurately recovers the distinct physical components in our simulated merger remnants. Other parameter choices may systematically fail (or fail in certain regimes of either high or low true starburst mass fraction) to recover a physically meaningful decomposition.

For example, Figure 2 also shows the results of fitting a pure Sersic function to the entire surface density profile (lower left). There is a reasonable fit to the entire profile with a single Sersic index $n_{s}=6.50$, very different from the Sersic index which describes either the pre-starburst or starburst light components. Likewise, if we consider the excess light to be that light in the real profile above the prediction of the best-fit Sersic model, we would infer only a tiny extra light fraction $\sim 0.5 \%$, essentially consistent with no starburst component (given the fit uncertainties). Although the fit is technically worse, with variance $\Delta \mu=0.19$, the difference is not dramatic, and by many observational standards would be con- 
sidered a good fit. Clearly, however, the fitted results have no physical meaning in this case.

The problem becomes even worse if we add a degree of freedom and fit a "cored Sersic" profile (lower right), of the form

$$
I=I^{\prime}\left[1+\left(\frac{r_{b}}{r}\right)^{\alpha}\right]^{\gamma / \alpha} \exp \left[-b_{n}\left(\frac{r^{\alpha}+r_{b}^{\alpha}}{r_{e}^{\alpha}}\right)^{1 /(\alpha n)}\right]
$$

(e.g. Graham et al. 2003), where $r_{b}$ is the core break radius within which the profile breaks to a power law of slope $\gamma$, $r_{e}$ is the effective radius and $n$ the Sersic index of the outer light profile, and $\alpha$ is a parameter describing how rapidly the break occurs. Figure 2 shows the results of this fit, which is again good in a purely statistical sense, albeit worse than our best fit Sersic+extra light fit $(\Delta \mu=0.16 \mathrm{mag})$. However, here the fit parameters become even more unphysical - the best fit Sersic index is a very steep $n_{s}=9.5$ and one actually infers that the system is a cored galaxy, with missing light relative to the best-fit Sersic profile.

The reason for these catastrophic failures is that the extra light component blends smoothly with the outer pre-starburst light profile. By increasing the central surface brightness, the extra light component makes the overall profile appear steeper (concave up in the $\mu-r^{1 / 4}$ projection), owing to the rise at small $r$. However, the cusp itself does not continue to rise steeply inwards (in most cases), so after steepening the bestfit Sersic index to fit the outer part of the extra light component, one is often forced to infer the existence of a core in the central regions. Again, these fits have no direct physical meaning, but they are not terrible matches to the light profile. This emphasizes that whenever fitting a parameterized profile to the data, one must take care to adopt a physically motivated procedure. Fortunately, there are some indications, from the purely observational point of view, that the Sersic only and cored Sersic fits are inappropriate (when applied in this manner and into the central regions of gas-rich merger remnants).

First, they begin to fail at large radii - however, this is where the true nature of the Sersic profile of the outer light component is most prominent, so any failure at large $r$ should be especially worrisome. Furthermore, when we examine the kinematics (e.g. ellipticity, boxy/diskiness, rotation properties) along the major axis, one can often see a transition occur in these properties where the extra light begins to dominate the profile (see $\S 7$ ), whereas one would expect no such change if a continuous Sersic profile was the meaningful choice. Finally, when fitting a cored Sersic profile in an inappropriate case, one often infers a large missing light fraction, relative to what is observed in genuine cored galaxies (e.g. massive, boxy, slow-rotating ellipticals; see Kormendy et al. 2007). One typically expects missing light masses in real cores of $\lesssim 1 \%$ the galaxy mass, comparable to the central black hole masses (e.g. Milosavljević et al. 2002).

Note that we are not saying that a pure Sersic or cored Sersic profile is always an unphysical parameterization of the galaxy light distribution - however, for gas-rich merger remnants, we know in our simulations and have good reason to believe observationally that there is some excess light component. In these cases, the results of these fits are demonstrably unphysical and can be misleading.

We caution that our adopted fitting functions and their physical interpretations are appropriate only for the galaxy spheroid, and should not be applied at radii where a galactic disk dominates the light profile. Implicitly, our choice of functional form also has the advantage of providing a check that stellar disks and distinct kinematic components do not contribute significantly to the surface brightness profile. Because it includes a Sersic and an exponential component, if a traditional disk plus bulge decomposition of the light profile were required (if such a decomposition were the best fit to the observed profile), we would see this in the fit - our intended "extra light" component would in fact dominate the light profile at large radii, with a larger effective radius and substantially lower effective surface brightness than the fitted bulge. We discuss these cases in more detail in Hopkins et al. (2008b), but note here that only a few of the simulated or observed systems show this behavior - in other words, they are primarily true ellipticals, not S0 galaxies (which should not be surprising, since they are primarily remnants of major $1: 1$ mergers). In our simulations, we can confirm from the stellar kinematics that such fits are only preferred when indeed a rotationally supported disk is a significant fraction of the light at large radii - i.e. so long as we restrict ourselves to considering galaxy spheroids, there is no ambiguity that our fits are physically distinct from and statistically superior to simple disk plus bulge decompositions. Ideally, in the cases where a traditional disk/bulge decomposition is required (i.e. there is a prominent large-scale disk), we would fit a three-component model: an outer exponential disk, and a two-component (dissipational and dissipationless) bulge. Unfortunately, even with the ideal data quality in our simulations, the degeneracies involved in fitting for three independent components are large, and in cases where a disk dominates the profile at large radii, there is no robust "lever arm" on the outer bulge profile shape. We therefore generally exclude these cases from our comparisons (although we still can and do compare these profiles directly to observed systems), but emphasize that including or excluding them from our simulation sample or the observed RJ04 sample makes no difference to our conclusions.

We also emphasize that although there are some superficial similarities between our adopted parametric profile decomposition and that in e.g. Côté et al. (2006) and Ferrarese et al. (2006), the two are in detail significantly different and address very different spatial scales and physical properties of the galaxies. Typically, the "outer profile" we refer to extends to and beyond (in our simulations) the limits of our ground-based photometry, corresponding to physical radii of $\sim 20-100 \mathrm{kpc}$, and our "inner profile" refers to the residual from a central starburst at scales where a significant fraction of the galaxy mass becomes self-gravitating (see $\S 9$ ), at $\sim 0.5-1 \mathrm{kpc}$. We stress again that we are not resolving inwards of the central $\sim 30-50 \mathrm{pc}$, and our modeling should not be extrapolated to within these radii without considerable care. In contrast, the "outer profile" in Ferrarese et al. (2006) is based on the HST ACS profiles, which extent to outer radii $\sim 1 \mathrm{kpc}$, and their "inner profiles" typically dominate the light profile at very small radii $\sim 0.01-0.02 R_{e}(\sim 10-40 \mathrm{pc}$ for most of their sample). This is more akin to separating our "inner" component itself into multiple sub-components - i.e. a starburst stellar component that blends (as we have shown) relatively smoothly onto the outer, violently relaxed stars and an innermost nuclear component. The authors themselves address this, and denote these nuclear excesses as central stellar clusters. Such systems may indeed be present (and could be formed in the same dissipational starburst which we model): but if so they are distinct subsystems sitting on top of the starburst light component, which we do not have the ability to 


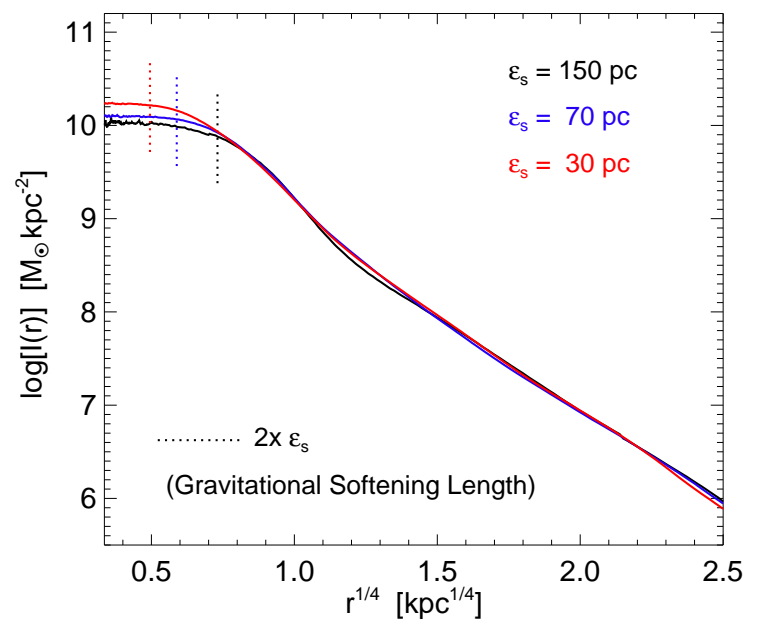

FIG. 3.- Surface brightness profile as a function of spatial resolution in a resolution study. The profiles are converged outside of roughly a softening length.

model or resolve in our simulations or the observations (the typical nuclear star cluster scales are well below the resolution limits of the observations from RJ04). Therefore, while the two approaches may yield complementary constraints and some similar conclusions, we caution that our results are not directly comparable and are specifically designed to trace distinct physical structures.

\section{THE EFFECTS OF RESOLUTION}

Given the small physical sizes of the starburst or extra light components in the simulations, it is important to verify that finite spatial resolution is not biasing our conclusions. In general, we have experimented with a wide range of simulation spatial and mass resolution scales and particle numbers, and find that all of the results in this paper (except where otherwise explicitly noted) are robust to the effects of resolution. However, we briefly present a detailed resolution study to examine these issues.

Figure 3 plots the mean (averaged over $\sim 100$ sightlines) surface mass density profile in a series of otherwise identical simulations with increasing spatial resolution, where the gravitational softening length decreases from $\approx 150 \mathrm{pc}$ to $30 \mathrm{pc}$. Note that the SPH gas smoothing length can be (and is) much smaller than this, especially in the dense, central regions of the galaxy during the starburst phase.

Unsurprisingly, the profiles flatten within several softening lengths. However, they are well converged at larger radii (all the differences between the mean profiles plotted at $>5$ softening lengths are within typical sightline-to-sightline variations). The primary difference seen in higher resolution simulations is that the profile continues to rise to smaller and smaller radii. Note that there is still some well-resolved curvature to the profile - the central extra light or starburst component is not rising as steeply as a pure power-law, for example. However, we expect from this trend that the profiles, with further improved resolution, should continue rising to small radii $\lesssim 30-50$ pc. Below these scales, we caution that, regardless of the numerical simulation resolution, our description of star formation and the ISM is no longer appropriate. Recall, we describe the ISM and star formation in a statistically volumeaveraged sense, representative of a balance between the multiphase components of the gas. By these smallest scales, we approach the physical sizes of molecular clouds and individual stellar clusters, and therefore cannot make physically
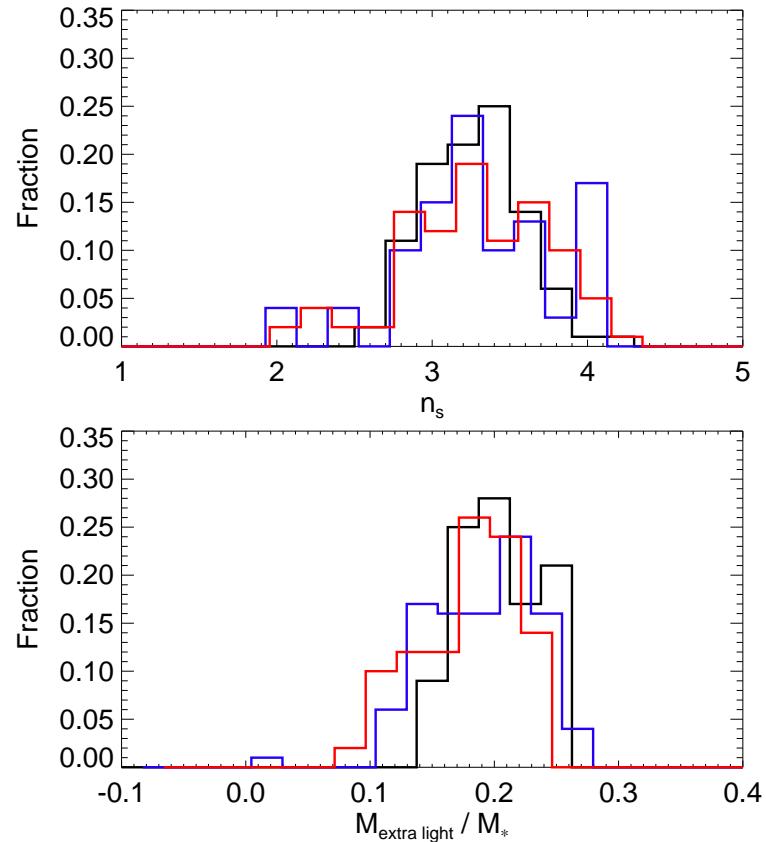

FIG. 4. - Distribution (across $\sim 100$ sightlines) of outer Sersic index and inner/extra light mass fraction fitted to the resolution study profiles in Figure 3 (black, blue, and red for $\epsilon_{s}=150,70,30 \mathrm{pc}$, respectively). The fits are reasonably well-converged even at relatively poor $(150 \mathrm{pc})$ spatial resolution.

meaningful claims without incorporating these gas phases and physics in a complete manner in the simulations.

Given these issues, it is clear that simulation resolution does affect the shape of the central regions of the extra light components, and hence we cannot make strong conclusions regarding their innermost shapes. However, we also see that the outer profile (the pre-starburst component) is well-converged, and that the simulations exhibit a transition to an extra light component at similar radii for all resolutions. The resolution limits do not affect the radii where the excess light begins to depart from the outer profile $(\sim 0.5-1 \mathrm{kpc})$, or most of the range of the observed $\mathrm{RJ} 04$ profiles with which we compare $(\gtrsim 100 \mathrm{pc}$ ). Figure 4 shows the distribution of Sersic indices fitted to the outer component of these simulations (across all sightlines), and the distribution of the fitted extra light component fraction. Within the errors, these distributions are more or less fully converged even for our lowest-resolution simulation. Similarly, if we directly extract the "starburst" and "pre-starburst" components from the simulations, we find that their total mass fractions, effective radii, and surface brightness profiles (outside a few softening lengths) are converged. We also show in $\S 8$ that the size-mass relation of the extra light components in the simulation is robust over the entire range of resolutions we have studied. We therefore conclude that resolution limitations, while constraining our ability to follow the profile of the extra light to the smallest radii, do not bias our overall estimates of the galactic structure or contribution of the extra light to the total mass.

\section{COMPARISON WITH OBSERVATIONS: THE EXTRA LIGHT IN LOCAL MERGER REMNANTS}

Bearing these cautions in mind, we apply our fitting procedure (calibrated from our simulations) to the sample of $\sim 50$ local merger remnants from RJ04 which spans a representative range in their post-merger stages (from LIRG and ULIRG systems still undergoing some star formation to shell ellipti- 

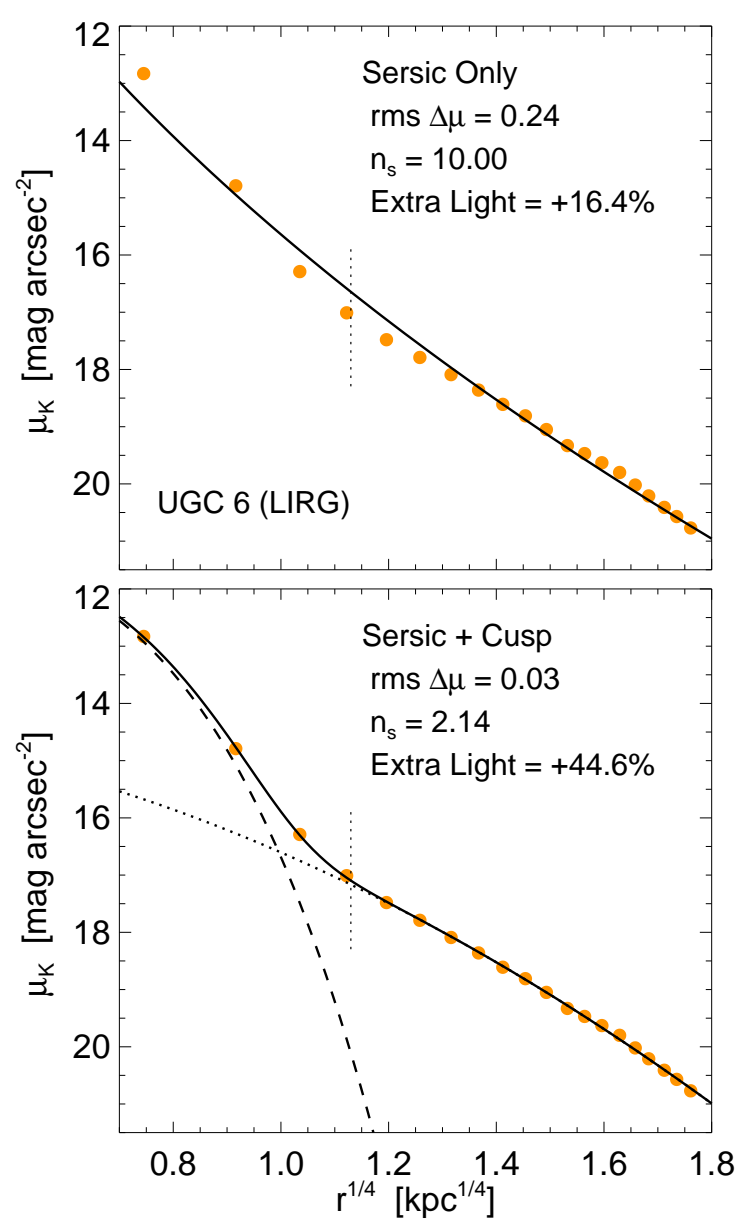

FIG. 5.- Observed $K$-band surface brightness profile (points) of the LIRG merger remnant UGC 6. Top: Single Sersic function fit (solid line) from RJ04, with the variance about the fit $\Delta \mu$, Sersic index, and extra light fraction. Dotted vertical line marks the half-light radius. Bottom: Our two component fit (total, inner, and outer as solid, dashed, and dotted). The fit quality is dramatically improved, and the outer Sersic index better describes the shape of the light profile at large radii.

cals which are completely relaxed).

We first illustrate the caveats from $\S 5$ with a representative example. Figure 5 shows the $K$-band luminosity profile of UGC6, a merger remnant and LIRG in the sample of RJ04. RJ04 fit their profiles to single Sersic laws, and their best fit is shown - they find a very large Sersic index $n_{s}=10$. RJ04 note that such a high Sersic value is probably unphysical, and parameters derived from it should be regarded with caution. Supporting this, Naab \& Truiillo (2006) note that collisionless merger remnants, which can be reasonably approximated by a single Sersic law, do not result in such large Sersic indices. RJ04 also make a first attempt to quantify the extra light in their objects by comparing the total luminosity to the inward extrapolation of a $r^{1 / 4}$-law (different from the bestfit free Sersic index fit), similar in spirit to our approach but with less allowance for the detailed structure of the inner and outer portions of the galaxy. It is clear that the observed profile indeed shows considerable structure not captured in this fit (overall it is rather poor, with $\Delta \mu=0.24 \mathrm{mag}$ ), and that the profile at large $r$ is in fact concave down in this projection (indicating $\left.n_{s}<4\right)$, not concave up $\left(n_{s}>4\right)$. Furthermore, the status of this object as a LIRG indicates that the merger must have been highly gas-rich; such gas-rich events are expected to produce more disk-like outer light profiles, not large Sersic indices.

Fitting instead to a Sersic+extra light component yields a much better fit in a statistical sense, even accounting for the added degrees of freedom $(\Delta \mu=0.03)$, as well as much more physical parameters - the outer Sersic index $n_{s}=2.1$ matches the concavity of the light profile and is expected in a gas-rich event, the profile is matched near the effective radius, and the shape of the extra light component is well-fitted. The resulting extra-light component is much larger with this fit, $\sim 45 \%$ (compared to $\sim 16 \%$ ) - this may seem high relative to what is typically quoted for such systems, but this has to do with our choice of fitting method. Indeed, such large fractional extra light components are reasonably common in simulations of very gas-rich mergers expected to produce LIRGs (see the example in $\$$ 4), and it is also observationally quite reasonable to see $\sim$ half the $K$-band luminosity of a LIRG originate in a relatively compact $\sim$ kpc nucleus (note though, as demonstrated in $\$ 10$, that the brightest LIRG and ULIRGs are the objects for which, even in $K$-band, there may be more extra light than extra mass, owing to stellar population effects).

Figures 6, 10 expand on these results by showing a comparison with a representative subset of the RJ04 sample. (For comparison with the entire RJ04 sample, see $\$$ A and Table 1 .) In each, we show the observed $K$-band luminosity profile, with our best-fit two-component model, and note the fitted outer Sersic index, extra light fraction, and variance about the best-fit. We also directly fit our simulations to the observed profiles - i.e. considering the surface brightness profiles of our entire library of simulations, and find those which match the observed systems most closely. Because our simulations sample a finite, discrete number of total galaxy masses, we allow the precise normalization of the simulation profiles to vary freely to match the observed data points (although we insist that the best-fit simulated galaxy mass be within a factor of $\sim$ a few of the estimated true galaxy mass, to make our comparisons as physically robust as possible). We show the three best-fit simulations to each profile (with the variance of the data about the profile), along with the Sersic index directly fitted to the pre-starburst stellar component of each and the mass fraction in the starburst component. We plot these components for the best-fit case. Errors refer to the range of a given parameter (e.g. $n_{s}$ of the pre-starburst component) in all simulations which are a comparably good match to the observations.

We also show $a_{4}$ and ellipticity profiles of observations and simulations, although we emphasize that these are not fitted, and exhibit much greater sightline-to-sightline variation than the surface brightness profiles (thus should be considered somewhat less robust measures of agreement). We caution that, as demonstrated in Cox et al. (2006b) and Naab et al. (2006), subtle features such as the isophotal shape $a_{4}$ are much more sensitive (compared to the surface brightness profile) to e.g. the orbital parameters and merger mass ratio. Furthermore, it is likely that seeing effects bias the observed ellipticity and $a_{4}$ near the resolution limit, and this may be significant to radii as large as $\sim 1 \mathrm{kpc}$; we also note that if the central regions have higher light-to-mass ratios owing to a nuclear starburst, they will appear more spherical than they truly are (although discrepancies in these shapes amount to small $\sim 0.1 \mathrm{mag} \operatorname{arcsec}^{-2}$ effects in the brightness profile). For example, the observed ellipticity profiles here all go to zero at small radii $\lesssim \mathrm{kpc}$, as do ground-based profiles of many nearby young or shell ellipticals - but in sufficiently high-resolution 

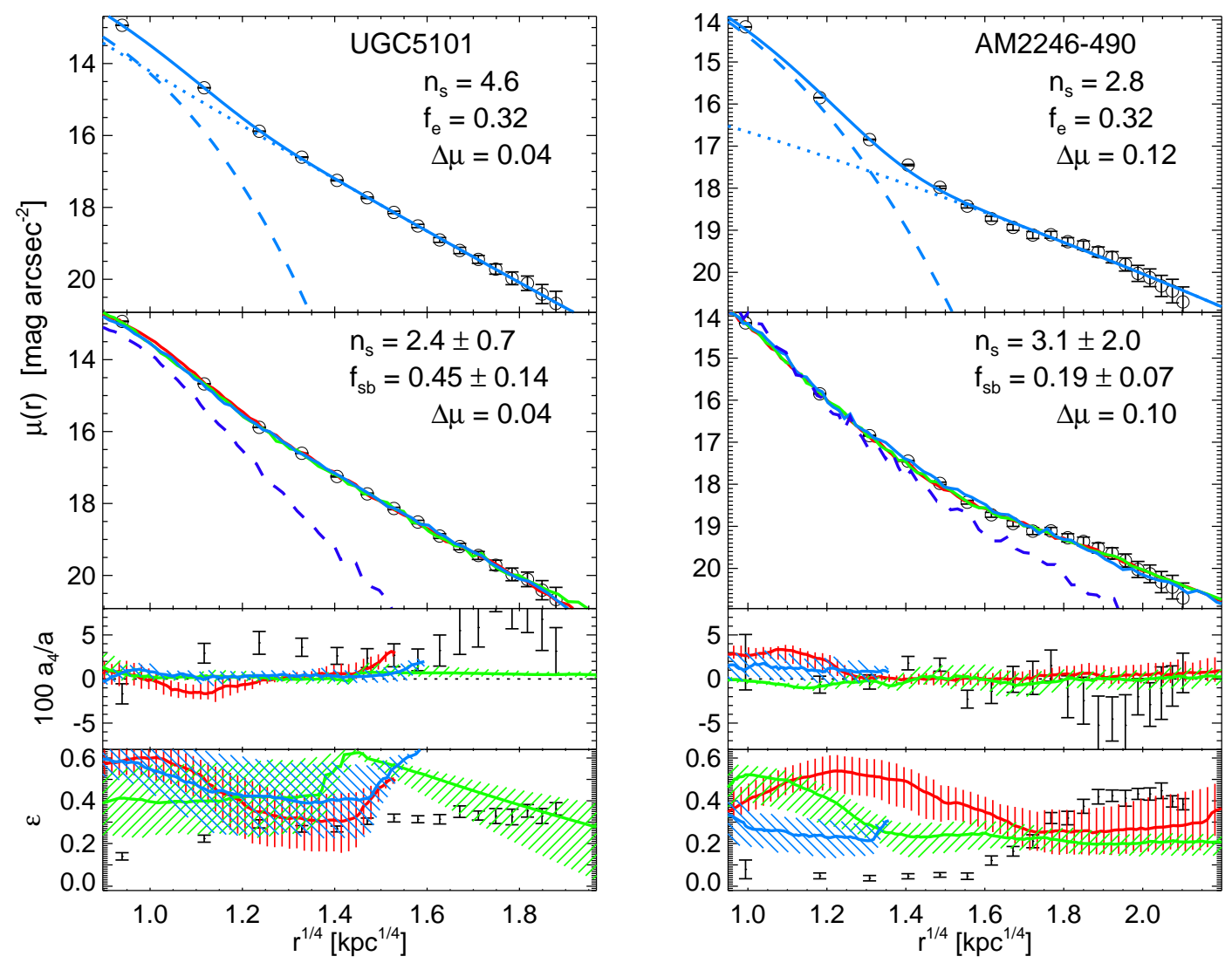

FIG. 6.- Observed, deconvolved major-axis $K$-band light profiles of gas-rich merger remnants from RJ04 (points with error bars). Top: Fitted two-component model (inner, outer, and total as dashed, dotted, and solid lines), with outer Sersic index $\left(n_{s}\right)$, extra light fraction $\left(f_{e}\right)$, and variance $(\Delta \mu)$ shown. Middle: Direct comparison with simulated surface brightness profiles. The three simulations which match most closely are shown (red, green, and blue lines from best to third-best fit). Dashed line shows the physical starburst component of the best-fitting simulation. Range in outer $n_{s}$ and physical starburst mass fraction $\left(f_{\mathrm{sb}}\right)$ for the best-fitting simulations are shown, with the variance of the observations with respect to the simulated profile. Bottom: Observed disky/boxy-ness $\left(a_{4}\right)$ and ellipticity profiles, with the median (solid) and $25-75 \%$ range (shaded) corresponding profile from the best-matching simulations above. Note that these are not fitted for in any sense. This panel shows the results for the ULIRGs, which require large gas contents and large extra light masses.

HST observations, it has been demonstrated that high ellipticities at $\sim \mathrm{kpc}$ scales continue (on average) at least down to $\lesssim 100$ pc (e.g. Lauer et al. 2005), similar to what is predicted in our simulations. Nevertheless, as is also seen in Naab et al. (2006), the agreement between the observations of RJ04 and our simulations is quite good at most radii, even in the isophotal shape and ellipticity profiles.

Both methods we have considered (fitting a parameterized profile and directly comparing to the simulations) have advantages and disadvantages - a parameterized fit is of course independent of the simulations, and conclusions can be drawn from it even if the simulation physics are incomplete. On the other hand, there is always bias introduced in some parameterized fit, and certain features in the light profiles (e.g. variation at large radii from shells or ripples, and embedded disks at intermediate radii) can throw off our fitting routines, but are accurately reflected in the direct simulation mass profiles. Reassuringly, in almost all cases the parameters describing the best-fit simulations are the same (within the errors) as those from our parameterized fits. The variance about the best-fit simulations is also comparable to that from the fitted profiles in a number of cases, the direct simulation profiles match the observed systems even better than a parameterized fit. This suggests that our fitting procedure is physically robust and that our simulations are reliably modeling real galaxy mergers and their extra light distributions.

\section{DEPENDENCE OF EXTRA LIGHT ON MERGER GAS CONTENT}

Having analyzed a large number of simulations and observed merger remnants, we can now study the properties of extra light or starburst components in a global sense. We expect based on our above analysis that the extra light should be a reasonable proxy for the central, dissipational starburst component, and therefore should scale with the gas supply of the merging galaxies. Figure 12 shows this explicitly for a systematic survey of gas fractions. The mergers are otherwise identical, and similar to our $\sim L_{*}$ fiducial case in $\S 4$ but vary in initial gas fraction from $f_{\text {gas }}=0.05-1$. We plot the sightline-averaged total surface brightness profile and median best-fit extra light and outer/pre-starburst Sersic components in each case. It is clear that the extra light component systematically increases with increasing gas fraction, as expected, typically containing $\sim 1 / 2$ the initial gas mass (although this exact value depends on a number of other conditions, as shown below). The profile of the scattered, violently relaxed stars, on the other hand, is basically independent of gas fraction, as expected if these are simply being scattered from a rotationally supported disk to a partially violently relaxed (Sersic-law $n_{s} \sim 2.5-3.5$ ) object.

Figures $13 \& 14$ extend this comparison to our entire suite of simulations and the fitted observations. We plot the extra light fraction as a function of the stellar mass (upper left) or initial gas fraction of the simulation (upper right). As expected, the mean extra light fraction increases with initial gas content, but there is a significant residual dependence on the galaxy stellar mass and orbital parameters. At fixed ini- 

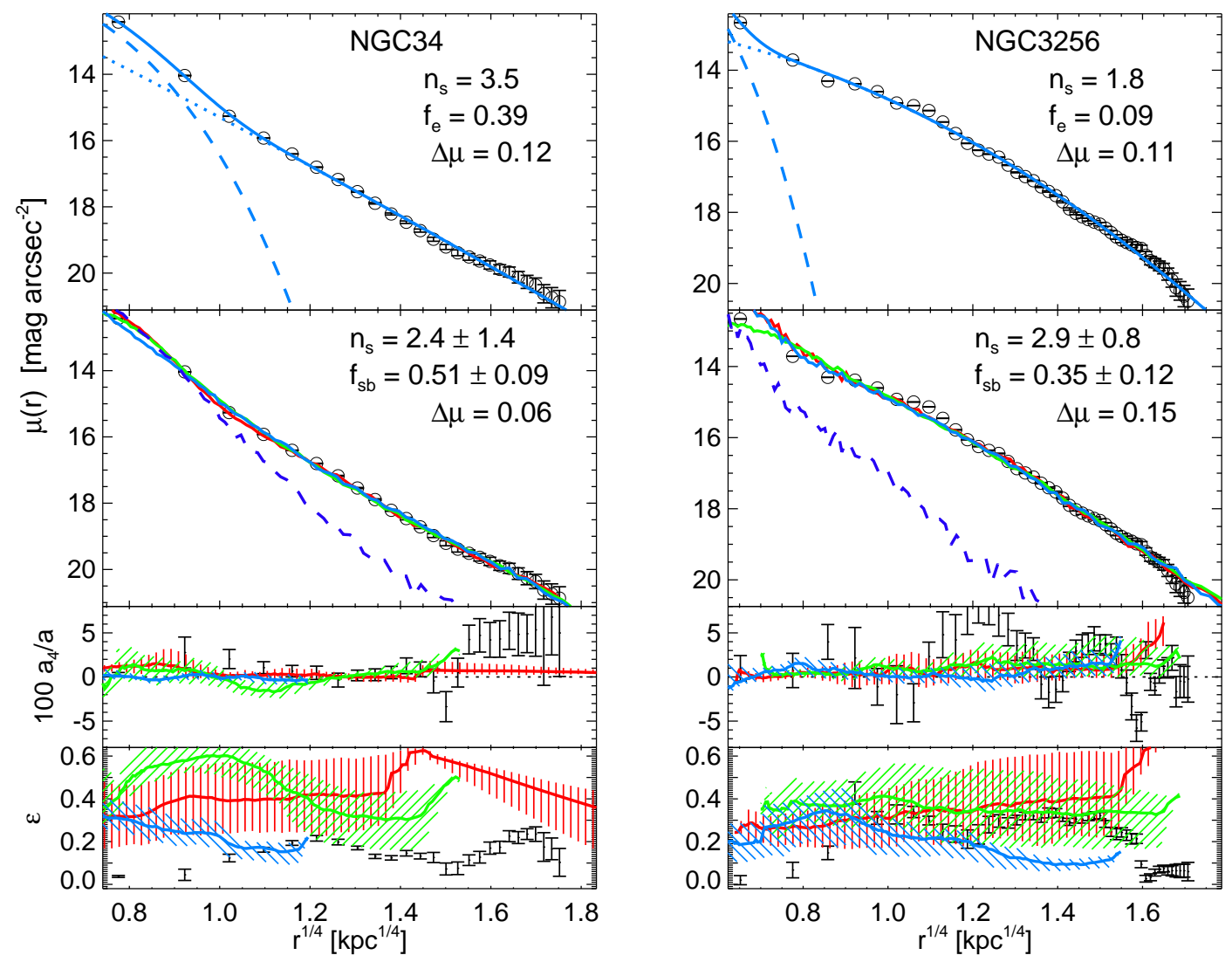

FIG. 7. - As Figure 6 Two LIRGs, which also require large gas content.
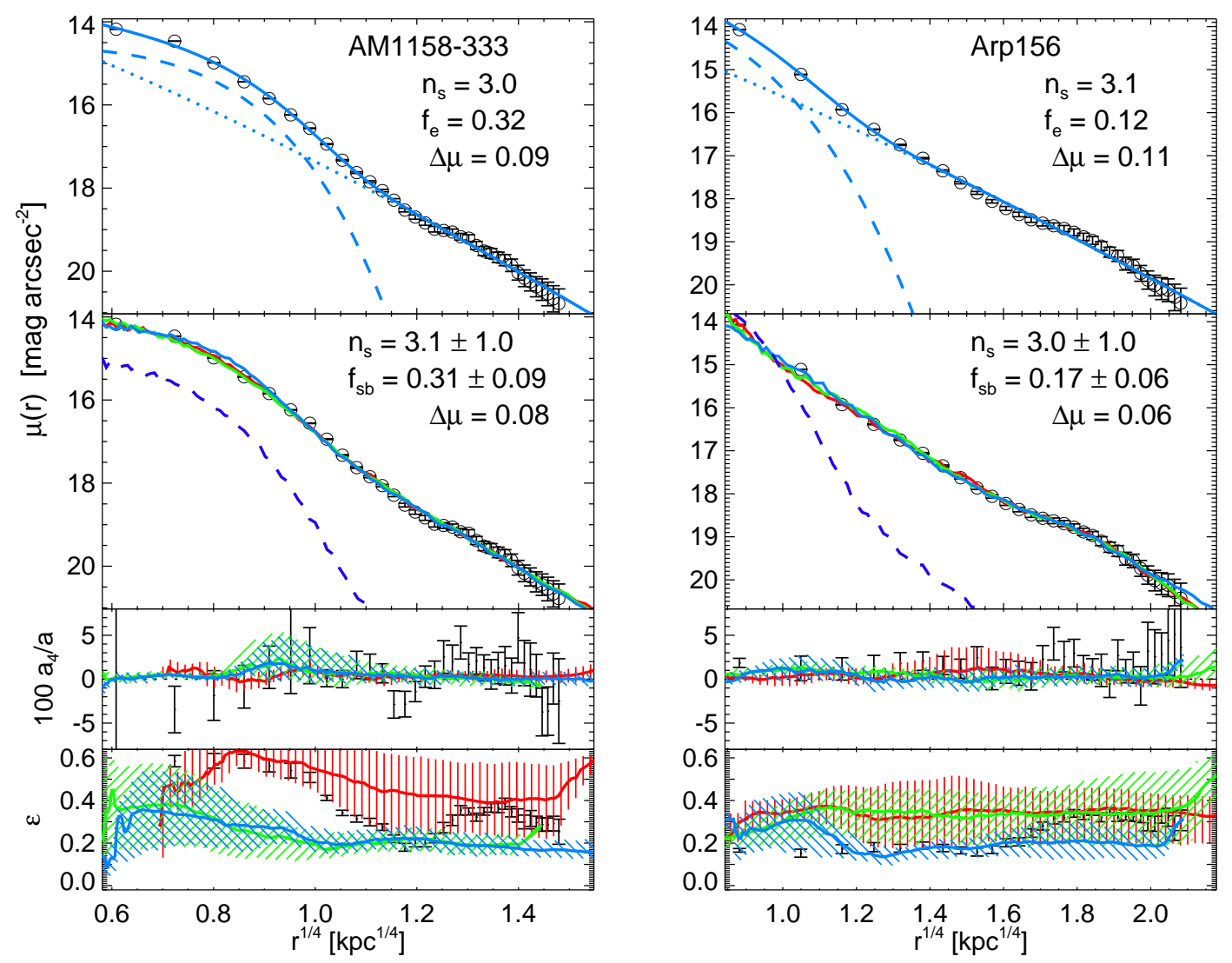

FIG. 8.- As Figure 6 Two merger remnants with very prominent (deviant from the outer Sersic law) central excess light components. 

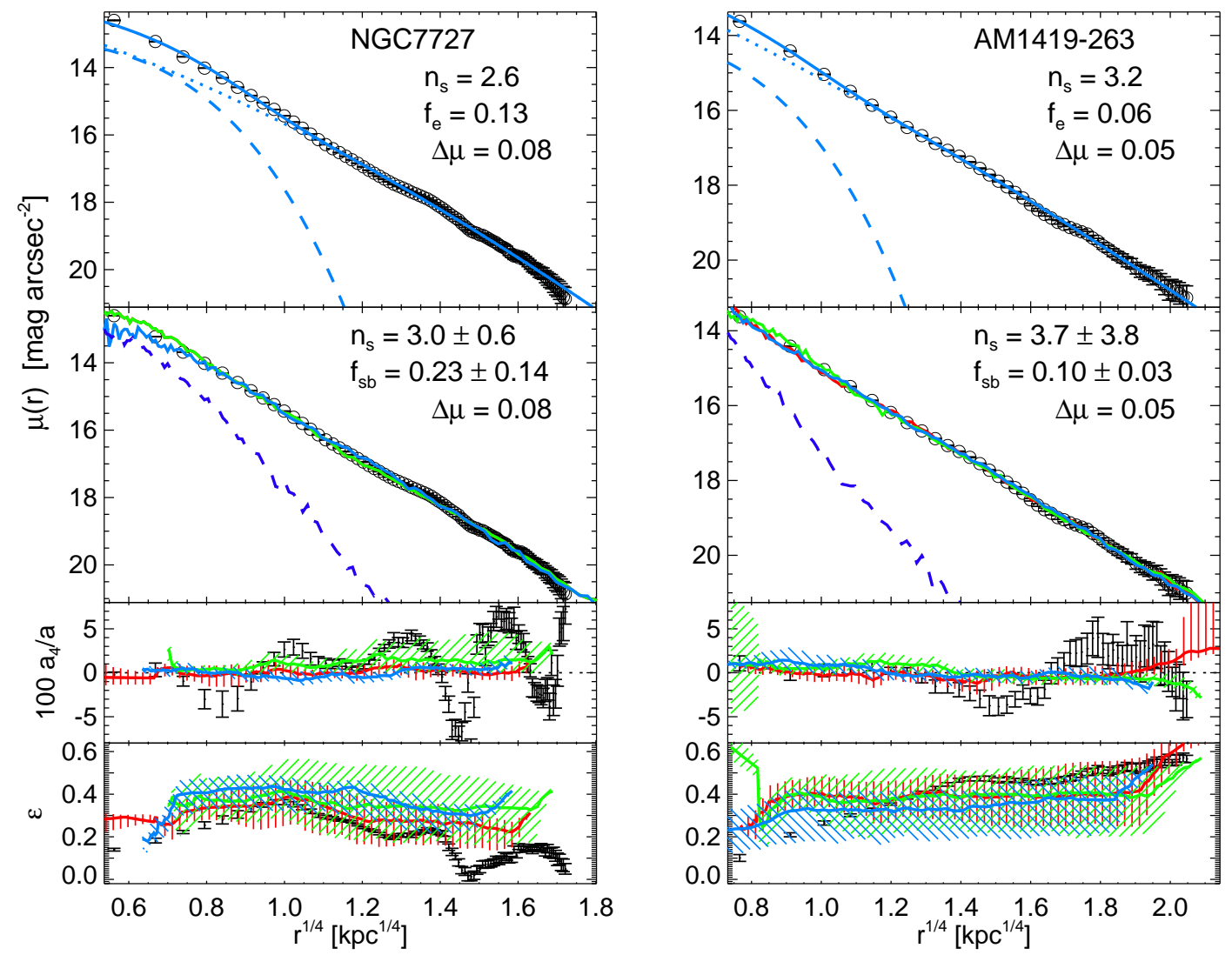

FIG. 9.- As Figure 6 Two merger remnants with less prominent central excess light components.
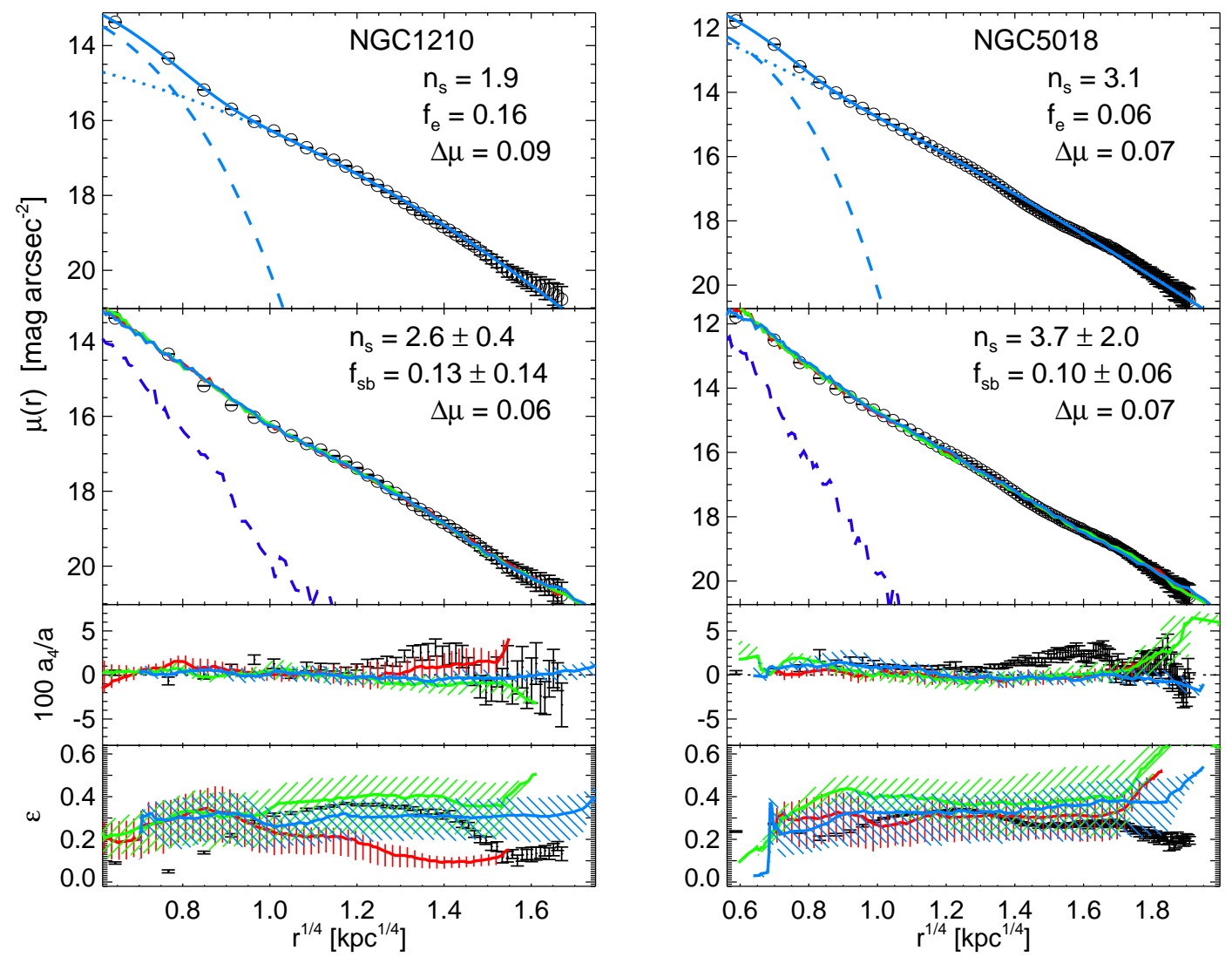

FIG. 10.- As Figure 6 Two shell ellipticals, with relatively low excess light fractions. 

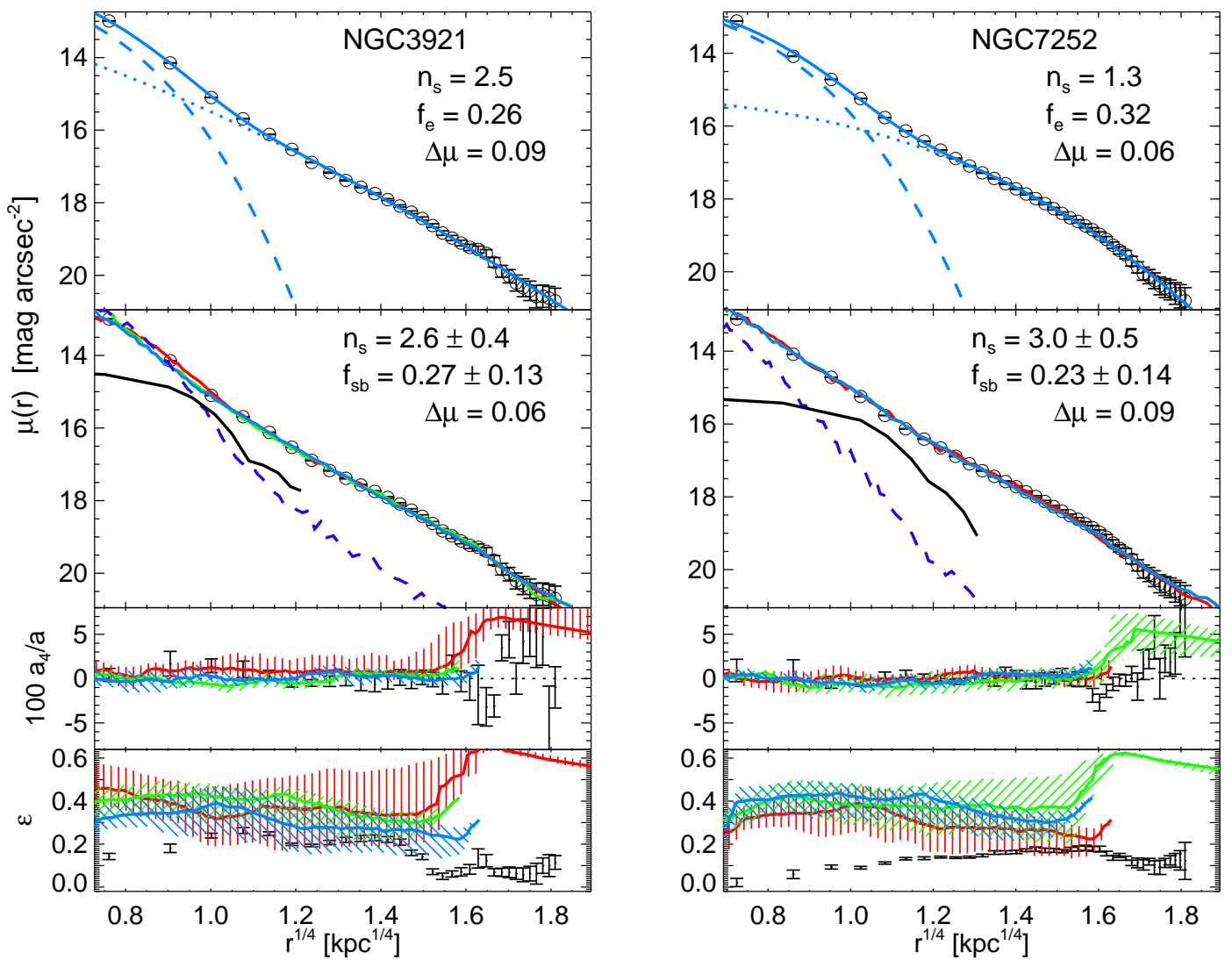

FIG. 11. - As Figure 6 Two merger remnants from the study of Hibbard \& Yun (1999). The central gas identified in that work is shown (black solid line in middle). These are remnants, so the central excess/starburst component as we identify it (and in the corresponding simulations) has already formed, and blends smoothly with the outer profile. The gas remaining corresponds to gas surviving the starburst (not part of the excess light), forming embedded disks (see Figure 11.

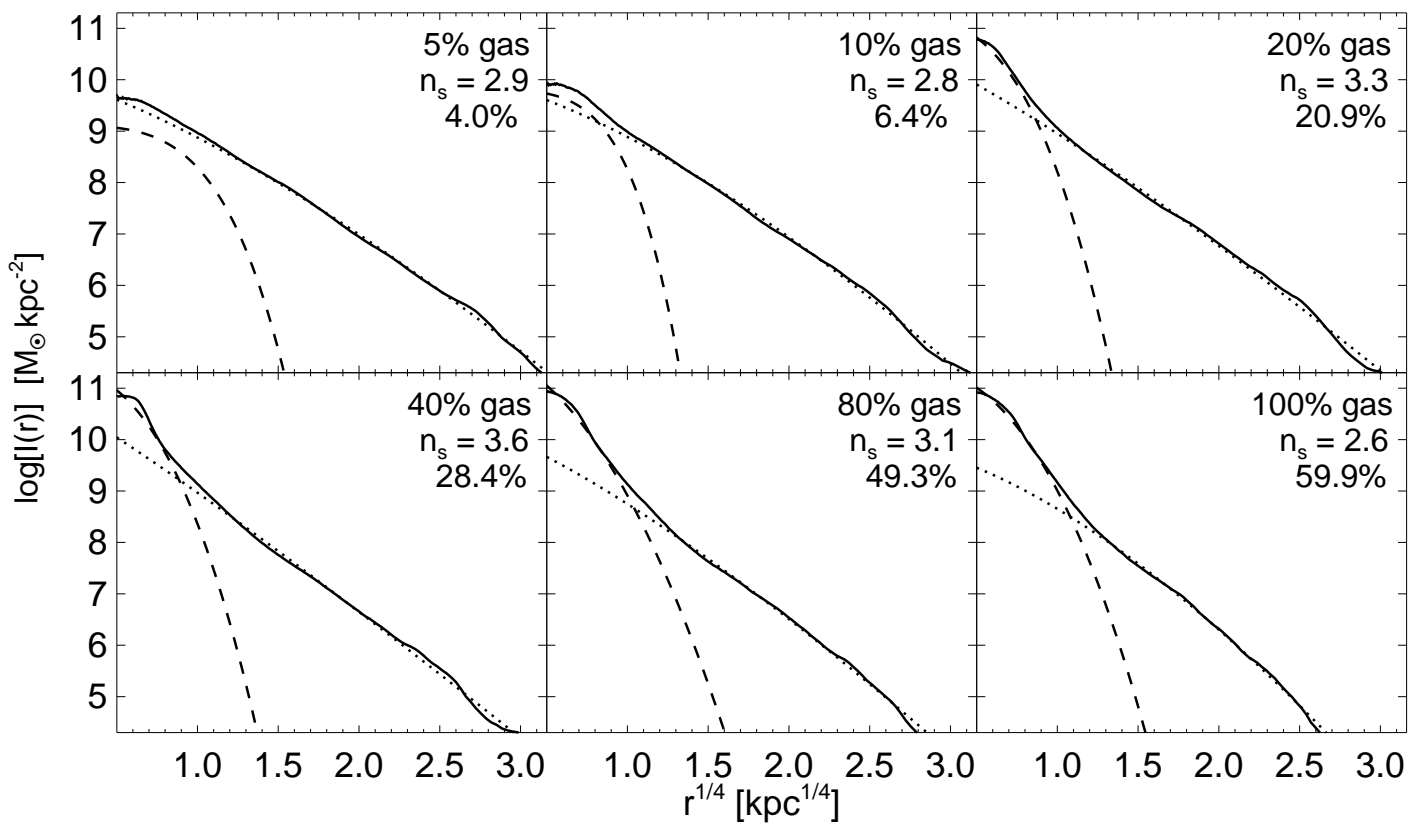

FIG. 12.- Median surface brightness profile (solid), with our inner (dashed) plus outer (dotted) component decomposition, of a typical simulation as a function of initial gas fraction. Outer Sersic index and extra light fraction for each are shown. All else being equal, the strength of the extra light component increases systematically with progenitor gas content. This does not necessarily mean that the excess light departs more sharply from the outer profile shape. 

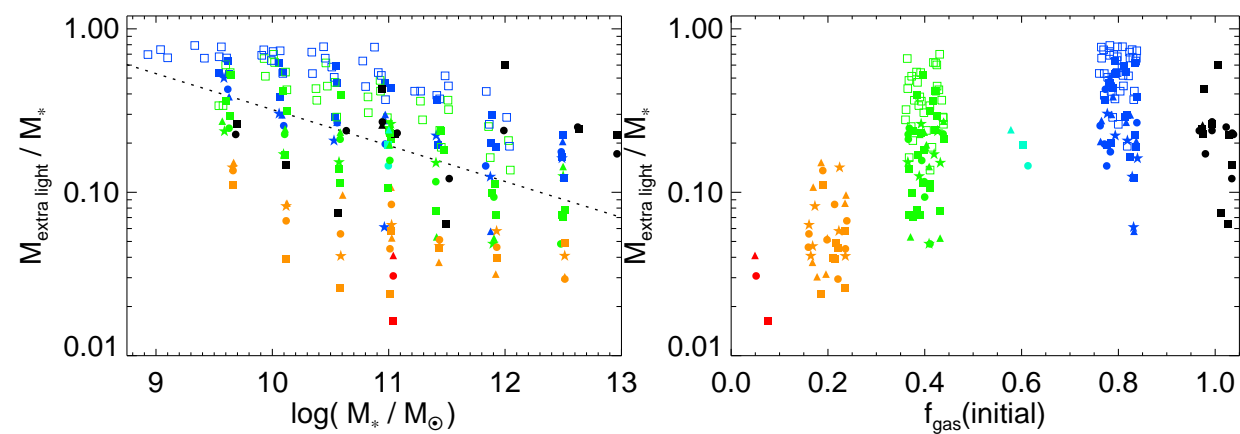

Gas Fraction:

- 0.1

- 0.2

- 0.4

- 0.6

- 0.8

- 1.0

Orbit:
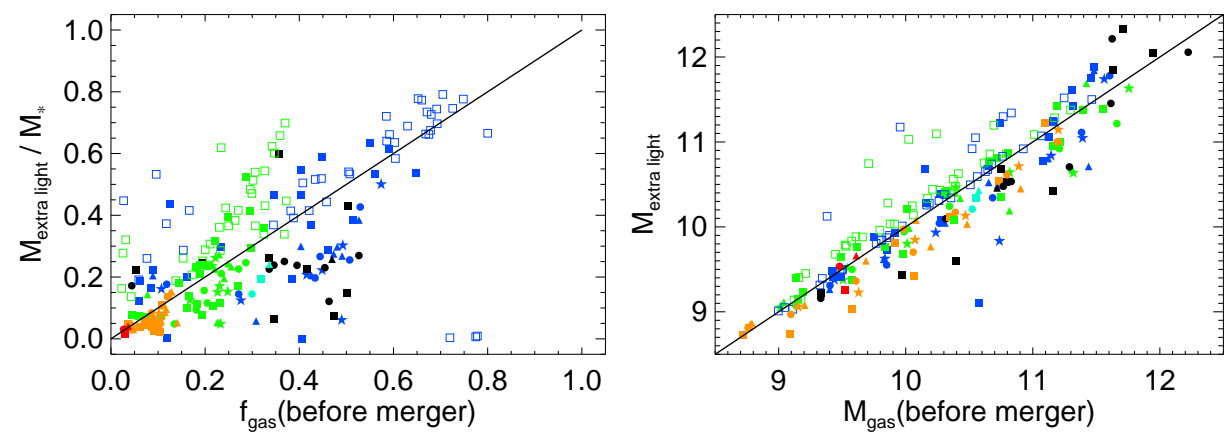

- Random

- Random (alt)

- Coplanar

$\star$ Polar

Initial Redshift:

- $z=0-2$

$\circ \mathrm{z}=2-6$

FIG. 13. - Fitted extra light fraction/mass as a function of stellar mass (top left), initial (simulation) gas fraction (top right), gas fraction involved in the final coalescence/merger (bottom left), and total gas mass in the final merger (bottom right). Shown are the results for simulations: color encodes gas fraction (red, orange, green, cyan, blue, and black for initial $\left.f_{\text {gas }} \sim 0.1,0.2,0.4,0.6,0.8,1.0\right)$, symbol shape encodes orbital parameters (circles and triangles are typical random orbits, squares coplanar, stars polar), and symbol fill encodes initial redshift (filled for $z<1$ typical progenitor disks/halos; open for $z>3$ compact progenitor disks/halos). The plotted simulation values are the median across $\sim 100$ sightlines; sightline-to-sightline differences are illustrated in Figure 4
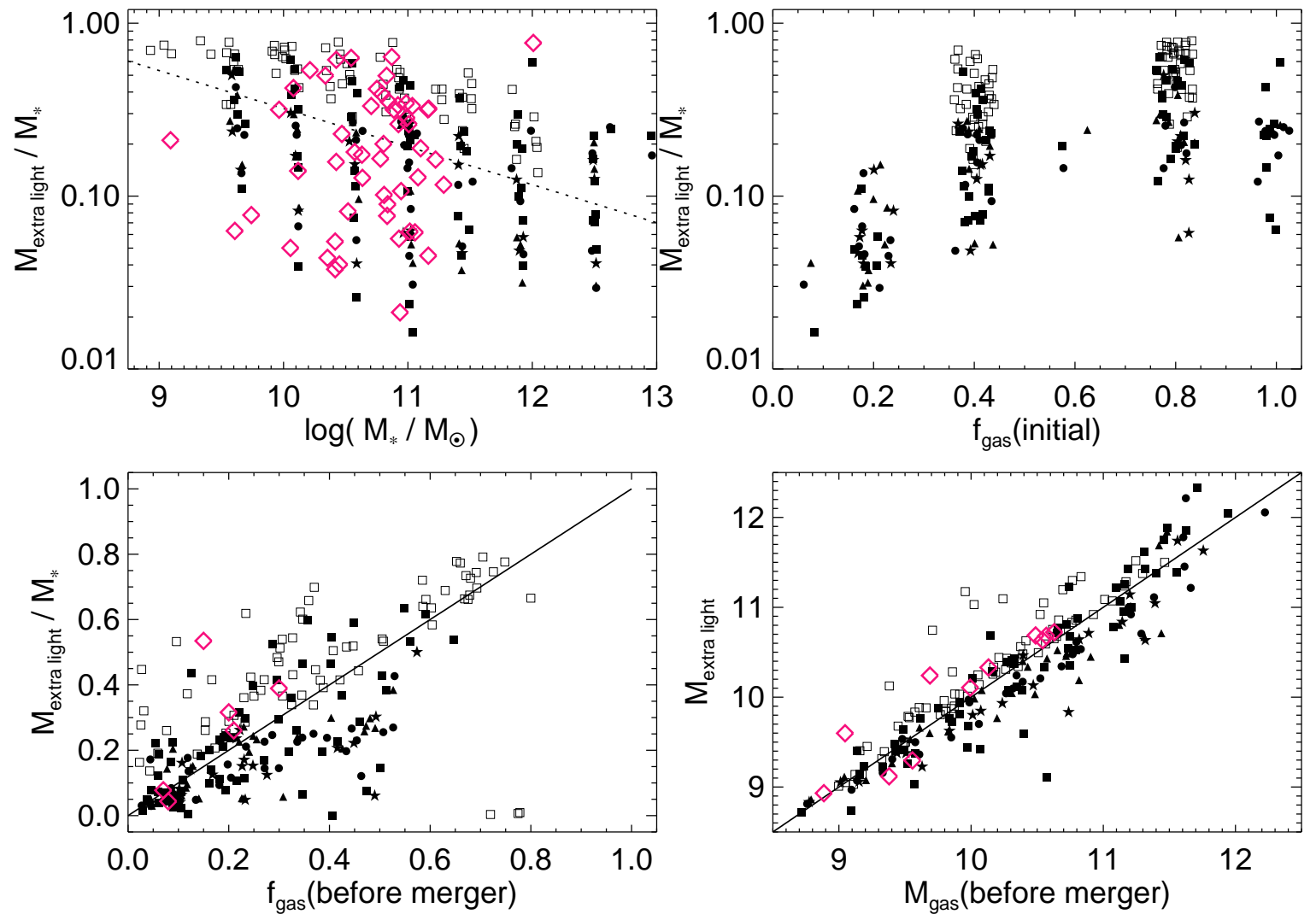

FIG. 14.- As Figure 13 but with the simulations shown in black and the observed systems from the sample of RJ04 (magenta diamonds). Where multiplecomponent stellar population analysis allows an estimate of the mass fraction formed in the merger-induced starburst, we have plotted the observed systems in the lower panels. 
tial gas fraction, the extra light fraction declines with stellar mass, in a roughly power-law fashion as $f_{\text {extra }} \propto M_{*}^{-0.15}$. As demonstrated by Robertson et al. (2006b), this is a consequence of the scaling of star formation efficiency with galaxy stellar mass. Higher mass galaxies have higher surface densities, and therefore exhaust their gas more rapidly - i.e. by the time the final merger and starburst occur, a typical $M_{*} \sim 10^{12} M_{\odot}$ galaxy will have a smaller remaining gas fraction available to participate in the compact starburst than a typical $M_{*} \sim 10^{10} M_{\odot}$ galaxy, even when both are initialized with the same gas fraction. Similarly, the orbital parameters determine how long it will take the galaxies to merge, and therefore how much of the gas will be consumed in the disks before the final merger event.

The physically relevant quantity is therefore not the gas fraction at the beginning of our simulations (which, as noted before, is somewhat arbitrary), but the gas fraction of the system just before the final merger/coalescence of the galaxies. If we consider the gas fraction $\sim 0.2 \mathrm{Gyr}$ before the peak of the final starburst or coalescence of the black holes (roughly the "beginning" of the starburst, in most systems), Figure 13 (lower panels) shows that there is a good, linear correlation between this pre-merger gas fraction and the extra light fraction in the remnant $\left(f_{\text {extra }} \approx f_{\text {gas, pre-merger }}\right)$. Accounting for this, there is no significant dependence on galaxy mass or orbital parameters. The only systems which deviate significantly tend to be pathological, either extremely gas rich $f_{\text {gas }}=1$, very high redshift $z=6$, or e.g. perfectly co-planar mergers. In all of these cases, the systems can retain a large amount of gas even after the merger and form relatively large new gaseous disks (Robertson et al. 2006a), which are not accounted for in our fitting. Even in these situations, the deviations are not large - plotting the total mass in the extra light component against the gas mass present just before the final merger shows a tight correlation, as expected.

Similarly, we find that changing properties of the simulations such as the presence or absence of an initial bulge, the concentration of the progenitor halos and disks, the presence or absence of a supermassive black hole, and the treatment of star formation and the ISM equation of state can all appear to change (albeit by a smaller amount than directly changing the gas fraction of the merger) the final mass in the starburst or extra light component. In all these cases, however, the effect is indirect - these choices influence how efficiently gas is consumed and/or expelled before the final merger, and therefore how much is available to participate in the starburst. For a fixed gas mass at the time of the final starburst, the extra light component mass is independent of these effects.

Figure 14 summarizes the results of our fitting to the entire sample of RJ04. We note that one should regard the stellar mass estimates (based just on the $K$-band luminosities) as somewhat uncertain - however, using dynamical masses from Rothberg \& Joseph (2006a) yields a similar result and using a more sophisticated stellar mass estimator makes little difference within the considerable scatter. The observed systems occupy a similar locus in the $f_{\text {extra }}-M_{*}$ plane (upper left) to our simulations. There are a couple with very low $f_{\text {extra }}-$ however, without exception, these also have rather low best fit outer Sersic indices $n_{s} \approx 1.0$, and are probably better classified as S0 or spheroidal galaxies, for which our fitting routines are not appropriate and the best-fit $f_{\text {extra }}$ is not necessarily physically representative.

We cannot, of course, assign a meaningful initial gas

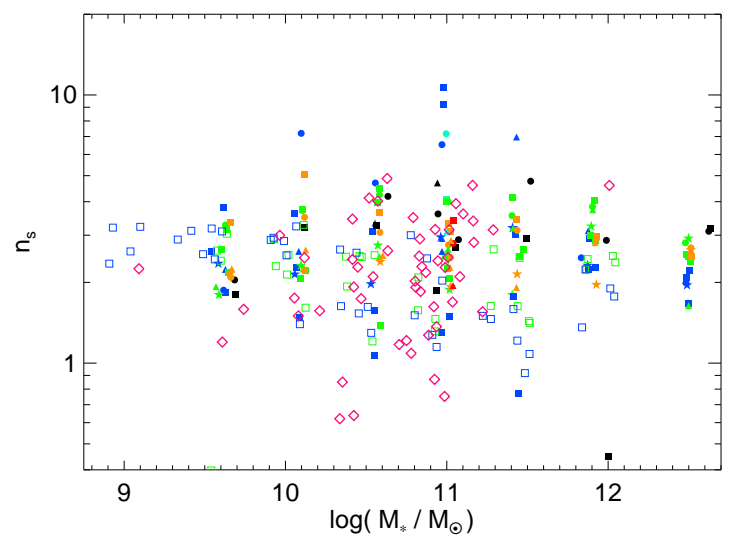

FIG. 15.- Outer Sersic indices in observed and simulated gas-rich merger remnants, using our two-component decomposition. Points are as in Figure 13 Gas-rich merger remnants have characteristic outer profile Sersic index $n \sim 2-3$, without a strong systematic dependence on mass or other properties.

fraction to the observed systems. However, it is in principle possible, by studying the stellar populations in sufficient detail, to estimate the mass fraction which formed in a recent, central starburst event (as opposed to the more extended quiescent star formation history). Unfortunately, there are still a number of degeneracies, and this requires detailed observations, but it has been attempted by Titus et al. (1997); Schweizer \& Seitzer (1998); Schweizer (1996); Schweizer \& Seitzer (2007); Reichardt et al. (2001); Michard (2006) for several of the observed systems. Adopting their estimates for the mass fraction in the newly formed stellar populations, we compare our fitted extra light fractions to the gas mass which participated in the starbursts in these objects. We find a similar tight correlation - although there are only a few objects for which sufficiently accurate stellar populations are available to allow this comparison, they all suggest that our fitted extra light component is indeed a good proxy for the mass fraction which was involved in the central, merger-driven starburst.

In Hopkins et al. (2008b), we compare the properties of progenitor disks required to form realistic merger remnants and ellipticals to the observed properties of disks, and find good agreement. Here, we briefly note that the implied gas fractions in Figure 14 agree reasonably well with those observed in local disks of the same stellar mass (for stellar masses $\sim 0.1-1 M_{*}$, where most of our sample lies, observations imply typical disk gas fractions $\sim 0.2$ at $z=0$ to $\sim 0.3$ at $z=1$, with a factor $\sim 2$ intrinsic scatter at both redshifts; Bell \& de Jong 2000; Shapley et al. 2005). Likewise, we obtain the fits to the observed surface brightness profiles in Figures 6.11 given progenitor disks that obey the baryonic TullyFisher and size-stellar mass relations (observed to evolve only weakly from $z=0-1$; e.g. Bell \& de Jong 2001; Flores et al. 2006; Truiillo et al. 2006). We have performed some limited studies of initial conditions which do not resemble observed disks (e.g. initial disks that are very compact for their mass), and find that these fare poorly at simultaneously matching the observed light profiles and stellar masses, or require unlikely gas fractions (see also Hopkins et al. 2008a). In short, the observed profiles are consistent with the expected remnants of typical local gas-rich mergers.

\section{STRUCTURE AND SIZE OF THE EXTRA LIGHT COMPONENT}


In Figure 15, we examine how the Sersic indices of the outer or pre-starburst light component vary with galaxy properties. Interestingly, there is no significant trend with galaxy mass. This appears contrary to the conclusions of Prugniel \& Simien (1997); Graham (2001); Ferrarese et al. (2006); however, we emphasize that we are fitting only gasrich major merger remnants. Their samples include a number of spheroidal galaxies and "pseudobulges" at low masses (at the lowest $n_{s}$ values) and cored, boxy, slowly rotating ellipticals at the highest masses (and highest $n_{s}$ values). To the extent that gas-rich merger remnants dominate the population at somewhat intermediate masses, the distribution of Sersic indices we recover is consistent with their estimated trends. Supporting this, Kormendy et al. (2007) find a similar, relatively constant distribution of Sersic indices if they consider just the elliptical galaxies in their sample which have some central excess light. Furthermore, our fitting procedure should not be directly compared to that in Prugniel \& Simien (1997) and others, because we fit an outer bulge and inner extra light component simultaneously, whereas they fit profiles to a single Sersic index.

Physically, this independence of $n_{s}$ on other parameters is expected - the gravitational physics which scatter the stellar disks in violent relaxation are self-similar. It is only the gas physics of dissipation and star formation, responsible primarily for the extra light component, that break this selfsimilarity. This is explicitly clear if we compare our simulations to those in Naab \& Trujillo (2006), who find that in simulated collisionless (gas-free) disk merger remnants - i.e. systems for which the entire profile is by definition part of the "outer," violently relaxed component - there is no significant dependence of the Sersic index on mass, effective radius, or merger mass ratio. At the lowest masses, some of our simulated remnants have very low Sersic indices $n_{s} \sim 1$ - these are generally low mass, extremely gas rich systems which form large disks after the merger, and should not therefore be considered typical spheroids.

We compare with the Sersic indices of the RJ04 sample. They occupy a similar locus and have a similar distribution to the simulations. There are a few objects with extremely low Sersic indices; as mentioned in $\S 8$ and Naab \& Truiillo (2006), these are probably systems that are actually diskdominated, and have small extra light components. For the systems that are genuinely bulge-dominated, the distribution of Sersic indices observed is consistent with the simulations. We also compare with the Sersic indices of relaxed ellipticals that have central cusps or extra light components, from Kormendy et al. (2007). These trace an almost identical distribution to the simulations and the RJ04 sample. Here, we do not see the anomalous objects with very low $n_{s}$ because the systems are specifically selected to be bulge-dominated. In both the observed merger remnants and ellipticals, there is no trend of $n_{s}$ with galaxy mass, as predicted by the simulations, a point discussed further in Hopkins et al. (2008b).

Figure 16 shows the full distribution of Sersic indices in the simulation outer bulge or pre-starburst components. The distribution is smooth and can be approximated by a simple Gaussian with median Sersic index $n_{s} \sim 2.6$ and $1 \sigma$ dispersion of $\Delta n_{s}=0.75$. The distribution of Sersic indices owes primarily to galaxy-to-galaxy variations: the figure shows the range of surface brightness profiles and fitted $n_{s}$ values for three random simulations, over $\sim 100$ sightlines. There is relatively little sightline-to-sightline variation in the surface brightness profile, and in general a small dispersion in Sersic index (dis-
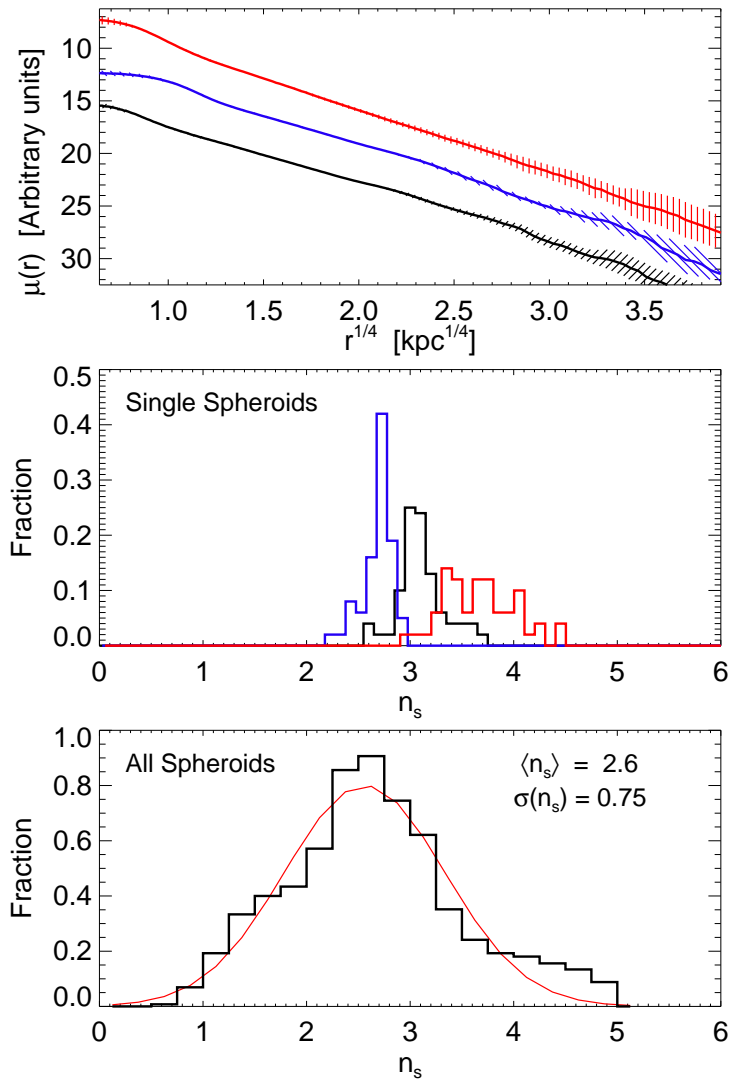

FIG. 16.-Top: Surface brightness profiles of three random $\left(\sim L_{*}, f_{\text {gas }} \sim\right.$ $0.2-0.4)$ simulations with slightly different $n_{s}$ values. Solid lines show the median profile over $\sim 100$ sightlines, shaded range shows the $25-75 \%$ range across sightlines. Middle: Distribution of fitted outer $n_{s}$ values for each of the simulations. Bottom: Distribution across all sightlines and simulations of outer Sersic index in our simulated gas-rich merger remnants. A Gaussian fit to this distribution is also shown.

persion of $\Delta n_{s} \sim 0.1-0.3$ across viewing angles). Again, this is similar to the simulated distribution in collisionless diskdisk merger remnants in Naab \& Truijillo (2006), which include a wider range in e.g. merger mass ratios, but do not include gas and therefore should be strictly self-similar.

For comparison with what is typically measured, Figure 17 shows the distribution of Sersic indices measured if we simply fit a single Sersic profile to the entire surface brightness profile of each of the simulated and observed merger remnants. Fitting a single Sersic profile, we see that the distribution of Sersic indices is systematically pushed to higher values, with a median $n_{s} \sim 4.0-4.5$ for both the simulations and observations, and a much larger "tail" towards high Sersic indices $n_{s}>5$. Fitted in this way, Sersic index does appear to depend (albeit weakly) on galaxy mass, as well as effective radius and surface brightness, similar to what has been seen in samples of ellipticals (when fit in this manner) as discussed above. We caution, however, that as demonstrated in Figure 2, these fits are not physically representative of the outer light profile or central extra light. The presence of an extra light component makes the total central light profile steeper, leading to the higher $n_{s} \sim 4$ typical of ellipticals that are fit to single Sersic indices - as compared to the $n_{s} \sim 2-3$ that genuinely characterizes the outer, violently relaxed stellar populations in our simulations. Because, as we have shown, this outer profile is roughly self-similar, the dependence of the single Sersic index on mass and other galaxy properties seen here owes to differences in the typical masses, shapes, and spatial extent of the 

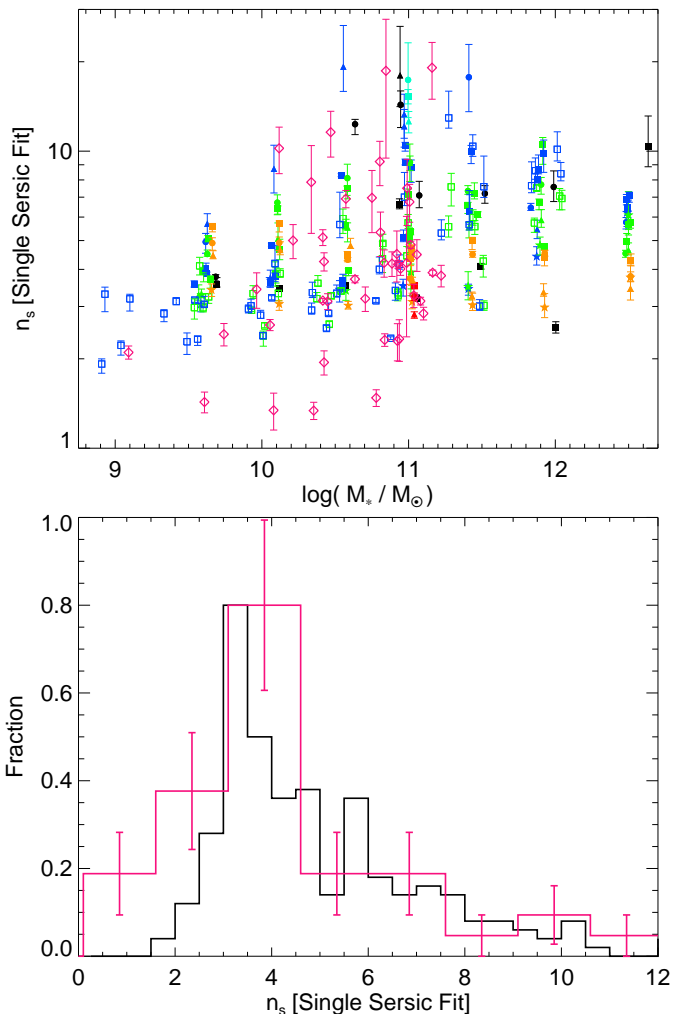

FIG. 17.- Top: Best-fit Sersic indices obtained from simulated and observed RJ04 merger remnants (style as Figure 15) when fit to a single Sersic profile (as opposed to our two-component fit). Bottom: Distribution of these single Sersic profile indices (histograms with and without error bars are for the RJ04 observed sample and simulations, respectively). Fitting to a single Sersic law yields higher Sersic indices and shows a dependence of $n_{s}$ on galaxy stellar mass, radius, and surface brightness similar to what is "typically" observed. As shown in Figure 2 however, this does not reflect the true shape at large radii (and should not be used to estimate e.g. the true extra light fraction), but rather indirectly reflects the contributions of extra light changing the profile shape at small radii.

extra light components, not to differences in the outer profiles. Even allowing for a fit to the full profile, RJ04 found (similar to what we see here) that the correlation between luminosity and Sersic index had a large scatter, and the correlations between e.g. size and Sersic index were even less significant. In short, the behavior of a single Sersic law fit in the simulations and observations is similar to what has generally been observed in elliptical galaxies, but when fit in this manner this "typical" behavior reflects a combination of the inner extra light structure (which depends on the degree of dissipation and therefore other galaxy properties) and outer, violently relaxed stars (which are approximately self-similar), rather than any single, robust physical component of the galaxy.

We can, in principle, also attempt to measure the Sersic indices specifically of the cusp or extra light components. Unsurprisingly, they typically show disky $n_{s} \sim 1-2$ profiles. However, we caution that the inner regions of the cusp are strongly affected by our resolution limits. It is guaranteed that near the resolution limit the surface density profile will become flat, which results in a lower Sersic index. Therefore, although the general trend that the extra light component tends to have a diskier profile than the outer light appears to be true in the observations as well, the structure in the inner regions of the simulated extra light components should not be considered robust. As discussed in $\S 5$, our choice to fit the inner extra light component to an $n_{s}=1$ law is not motivated by the actual shape at small radii (well inside the effective radius of the extra light), which neither simulations nor observations resolve (and high-resolution observations of ellipticals suggest can have irregular structure owing to e.g. star clusters and the effects of black hole dynamics; see e.g. Lauer et al. 2005); instead, we chose this because it fits reasonably well to where the extra light maps on to the outer profile and provides the most reliable recovery of the true physical starburst component.

More robustly, we can study the effective radii of the extra light or starburst components in the simulations and observations. Figure 18 compares the spatial extent of the extra light component to the properties of the galaxy. The extra light component appears to be fractionally smaller in more massive galaxies (relative to the effective radius of the galaxy), in both simulations and observations. Looking at this in detail, it is clear that this is a consequence of the trend in Figure 13 - more massive galaxies tend to have less mass in their extra light components. The extra light itself appears to follow a rough size-mass relation, shown in Figure 18 of the form $R_{e, \text { extra light }} \propto M_{\text {extra light }}^{0.33}$. Note that by size of the extra light component, we refer explicitly to its half-mass projected effective radius: this is not necessarily the same size scale at which the extra light component begins to dominate the total surface density profile of the galaxy. The appropriate comparison is with the effective radii of the extra light components fitted to the observations - where we see good agreement. By mass of the extra light component, we simply refer to our fitted extra light fraction times the total estimated stellar mass of the galaxies.

Considering the evolution of the starburst component in the simulations, it appears to follow a general formation mechanism, as outlined in Mihos \& Hernquist (1996). In the final stages of the merger, the gravitational torques acting on the gas remove its angular momentum, and it essentially begins a free-fall towards the central regions of the galaxy. The cooling time of the gas is almost always shorter than the dynamical time at this stage, so the process is not pressure supported and is primarily a gravitational collapse. This continues until the collapsing gas becomes self-gravitating: at this point, the gas shocks and establishes a quasi-hydrostatic central mass concentration, and becomes stable against further collapse. The stability criterion is simply that the effective equation of state of the gas be $\gamma>4 / 3$, which is true at this time because the gravitational compression of the gas is effectively adiabatic. Furthermore, in the classical McKee \& Ostriker (1977) picture of the multiphase ISM, the densities are sufficiently high that the system is subject to thermal instability. In this state, the hot and warm diffuse phases of the ISM can radiate and cool efficiently into cold, dense clouds, which then form stars, whose supernovae and stellar wind feedback reheat the diffuse ISM. The important point is that, in this regime, cooling does not allow the system to lose effective pressure support, because the cooling of the diffuse ISM is balanced by energy injection from star formation and supernovae in the cold phase of the ISM. Regardless of the cooling time, then, one expects that the system cannot collapse further once it becomes selfgravitating, and will stall at this radius and continue rapidly forming stars until the gas supply is exhausted.

Studies of the central regions of starburst systems support this picture - typically, observations find a dense concentration of molecular clouds forming stars at a high rate, with very high effective temperatures and pressures of the 

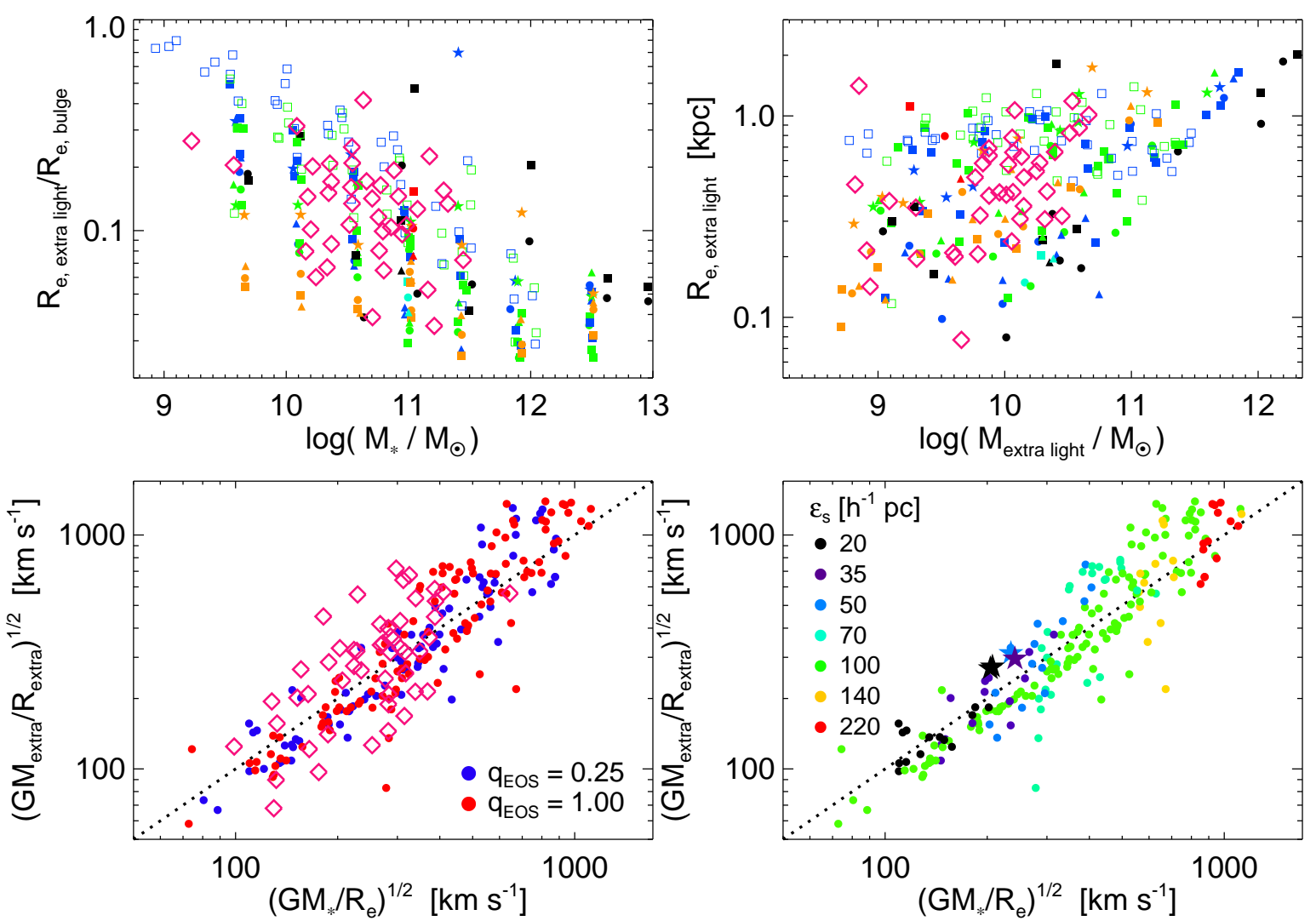

FIG. 18.- Top Left: Ratio of the half-light radius of the fitted extra light component to that of the fitted outer Sersic component, as a function of galaxy stellar mass. Points show simulations and observations, as in Figure 13 Top Right: Size-mass relation of the extra light. Bottom Left: Potential of the extra light component (here defined as $\sqrt{G M / R}$ ) as a function of that of the entire galaxy. Dotted line denotes a linear relation (indicating that the extra light is self-gravitating). In this space, there is no systematic dependence of extra light component size on e.g. galaxy mass, orbital parameters, or merger redshift. Colors here denote the effective ISM pressurization and equation of state: red for the full (Springel \& Hernquist (2003) model, blue for a nearly isothermal model. Bottom Right: Same, but colors denote simulation spatial resolution, from $\sim 300 \mathrm{pc}$ to $\sim 30 \mathrm{pc}$. Stars denote the resolution study simulations from Figure 3

cumulative (diffuse) ISM gas (e.g. Sanders \& Mirabel 1996; Solomon et al. 1997; Bryant \& Scoville 1999). Even if the system can contract, it does so on the local dynamical time, which, for a self-gravitating system, is comparable to the star formation timescale if the system obeys a Kennicutt-Schmidt type star formation law (Kennicutt 1998), and so the system will exhaust gas in star formation in a self-similar manner as it contracts.

The expectation of these physical models is then that the effective radii of the extra light components will be determined by the condition that they become self-gravitating:

$$
\frac{G M_{\text {extra }}}{R_{\text {extra }}}=\alpha \frac{G M_{\text {tot }}}{R_{e}}
$$

where $\alpha \sim 1$ is a constant which depends on the exact shape of the galaxy mass profile and stellar-to-dark matter mass ratio as a function of radius. Figure 18 plots this relationship, for both the simulations and the observed sample of RJ04. We find that indeed, the objects follow a tight, linear correlation as predicted, with a best-fit normalization $\alpha \approx 1$, independent of the absolute value of galaxy mass, orbital parameters, or the initial redshift of the merging galaxies.

If the spatial extent of the extra light component were not set by the self-gravitation condition, but by, for example, the pressure support of the ISM or some competition between the star formation and cooling timescale, one would expect the size to be systematically sensitive to the treatment of star formation and supernova feedback and the corresponding ISM equation of state. Figure 18 compares the size-mass relation for two values of our equation of state parameter $q_{\mathrm{eos}}$, described in $\$ 2$. Our fully multiphase model for the ISM, with a high effective pressure, corresponds to $q_{\mathrm{eos}}=1$, and a model much closer to a completely isothermal ISM to $q_{\mathrm{eos}}=0.25$. Both cases follow an identical size-mass relation, suggesting that it is indeed simply the self-gravitation condition that sets the size of the starburst component. Note that the absolute sizes of the extra light or starburst components tend to be smaller in the $q_{\text {eos }}=0.25$ case. This is because the more isothermal equation of state allows star formation to proceed more efficiently in the pre-merger disks, exhausting more of the gas supply before the final merger and leaving an extra light component of lower total mass. But, for fixed gas mass just before the final merger stages or (equivalently) fixed starburst mass fraction, the two equations of state yield extra light components of the same physical size.

It is also natural to wonder whether or not the sizes of the cusps are sensitive to our simulation resolution. Figure 18 compares the points on this effective size-mass relation as a function of the simulation resolution. From our lowest resolution simulations with gravitational softening length $\epsilon_{s} \approx$ $300 \mathrm{pc}$, to our highest resolution simulations with $\epsilon_{s} \approx 30 \mathrm{pc}$, the systems obey the same correlation. This is reassuring in that our resolution limits, while such that we do not resolve the inner regions of the extra light, do not cause us to systematically overestimate the size of the extra light distribution. We have also tested a series of simulations of varying integra- 


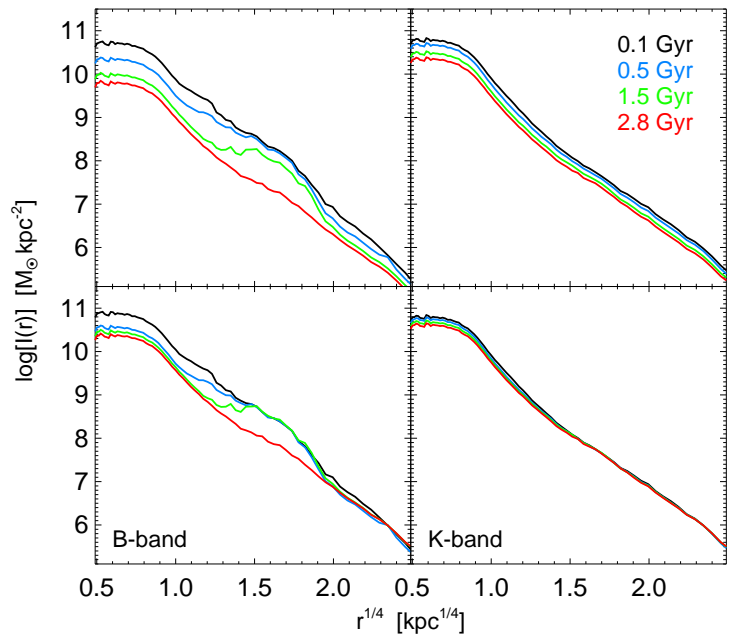

FIG. 19. - Surface brightness profile of an extremely gas-rich $\left(f_{\text {gas }}=0.8\right)$ merger remnant in $B$-band (left) and $K$-band (right), as a function of time after the coalescence of the two merging nuclei (colors, as labeled). Top: Absolute values, which show fading owing to passive evolution. Bottom: Profiles rescaled to the same value at $\sim 30 \mathrm{kpc}$, to show the change in profile shape. There is little evolution in $K$-band, but substantial time dependence in $B$-band. In this case the system forms an embedded disk (around $t \sim$ $0.5 \mathrm{Gyr}$ ) from gas which survives the merger, which is prominent in $B$-band (the "bump" at $\sim 4-15 \mathrm{kpc}$ ) owing to its young age but negligible in $K$ band (it contains only $\sim 4 \%$ of the galaxy stellar mass). The young stellar populations in the embedded disk have faded in $B$-band by $\sim 3 \mathrm{Gyr}$.

tion accuracy, and find similar results.

\section{STELLAR POPULATIONS AND EVOLUTION OF EXTRA LIGHT} COMPONENTS WITH WAVELENGTH AND TIME

Figure 19 plots the evolution of the surface brightness profile of a highly gas-rich merger (initial $f_{\text {gas }}=0.8$ ) remnant with time, in the observed $B$-band and $K$-band, ignoring (for now) the effects of dust obscuration. We calculate the observed surface brightness profile in these bands using the stellar population synthesis models of Bruzual \& Charlot (2003), given the ages and metallicities determined self-consistently for the stellar particles formed in our simulations, assuming a Chabrier (2003) IMF (although this choice primarily affects only the total luminosity, not the shape of the light profile). The initial stars in the simulation disks (in this case a relatively small fraction $1-f_{\text {gas }}=0.2$ ) are given premerger age, metallicity, and enrichment distributions appropriate for the disk stellar mass according to the median bestfit $\tau$ model star formation histories fit to local observed disks in Bell \& de Jong (2000) (thereby are guaranteed to lie on the observed star-forming galaxy mass-metallicity relation). We discuss the issues of stellar population gradients in much greater detai in Hopkins et al. (2008b), but note that we have also experimented with other stellar population models or fitted star formation histories, and with including initial gradients in the disk stellar populations, and find these choices make little difference (the central age, metallicity, and gradients of interest are largely set by the dissipational starburst).

The figure demonstrates that, although there can be significant overall fading in the near IR with time after the merger (in this case $\sim 2$ magnitudes over $\sim 3 \mathrm{Gyr}$ ), especially for such a gas rich system where a large fraction of stars are formed in the final starburst, this has little differential effect with radius. The shape of the surface brightness profile in $K$-band is nearly time-independent, after the final coalescence of the two galaxies. In optical bands such as $B$, however, there can be a much more dramatic time dependence. In this case, not only does the nucleus fade considerably relative to the outer profile, but one can see a prominent embedded disk appear at $\sim 0.5 \mathrm{Gyr}$ and fade out by $\sim 3$ Gyr after the merger.

Because the system is so gas-rich, a significant quantity of gas survives the merger and cools to re-form an embedded disk. This process takes $\sim$ a couple $\times 10^{8} \mathrm{yr}$ after the coalescence, hence the disk is not as prominent immediately following the merger. By mass, the disk represents only $\sim$ a few percent of the galaxy stellar mass, similar to the case in Figure 1 However, because its stellar populations are very young, it appears prominently in $B$-band, constituting $\sim 30-40 \%$ of the total light and making the system appear to be a clear S0 rather than a true elliptical. By $\sim 3 \mathrm{Gyr}$ after the merger, the embedded disk (while still forming stars at a low rate) has aged sufficiently so that its mass-to-light ratio is not much different from the rest of the galaxy, and (given its small mass fraction, which is concentrated near $R_{e}$, where most of the spheroid mass also lies) it disappears again. Note that, to illustrate this, we have ignored dust, which will preferentially lie in the gas-rich star forming region of the disk, and make the difference between $B$ and $K$ bands less obvious. Still, the presence of such disks in optical (and not NIR light) is not uncommon in gas-rich merger remnants: the profile and evolution above is similar to that seen (and inferred) for NGC 34 (Schweizer \& Seitzer 2007).

The example above and observed systems emphasize that, at times close to the merger, the $K$-band is a much more robust tracer of the stellar mass distribution. Our experiments suggest that we can have reasonable confidence that the $K$-band profiles of the observed systems will not change substantially with time - therefore our results would likely be unchanged if we analyzed the merger remnants at a time when they were more relaxed. Of course, then, it would not be clear that the systems are indeed gas rich merger remnants. The most substantial change would probably be a reduction in the scatter about the mean trends defined in Figures 13 and 18, we typically see little evolution in the median surface brightness profiles after the merger, but unrelaxed features can cause considerably larger sightline-to-sightline variation.

Figure 20 shows the evolution with time of the best-fit surface brightness profile parameters for two more representative cases, one with a large initial gas fraction and one with a small initial gas fraction. Unlike Figure 19 which was just a qualitative illustration, we include dust obscuration by calculating the column densities through the gas in our simulations in the manner of Hopkins et al. (2005a b). We show the evolution of the absolute effective radius (i.e. independent of any fitting), the best-fit outer Sersic index, and the best-fit extra light fraction with time after the final coalescence, in terms of stellar mass, $K$-band light, $B$-band light, and $B$-band light in the absence of dust obscuration.

The effective radius and extra light fraction in terms of stellar mass show little evolution with time. In other words, the profile is relaxed out to $\sim R_{e}$ in a short time ( $\sim$ the dynamical time). The behavior in $n_{s}$, however, is more sensitive to the outer regions where the dynamical time is long, and therefore more varied. We see in Figure 20 that, in one case, $n_{s}$ declines slowly with time - this is caused by a large sloshing from the merger, which moves stars to large radii and forces a fit with a more extended envelope (higher $n_{s}$ ), until the sloshing relaxes. In the other case, there is a clear ringing or oscillation in $n_{s}$. This is caused by material on nearly radial orbits creating shells, as described in detail in Ouinn (1984); 


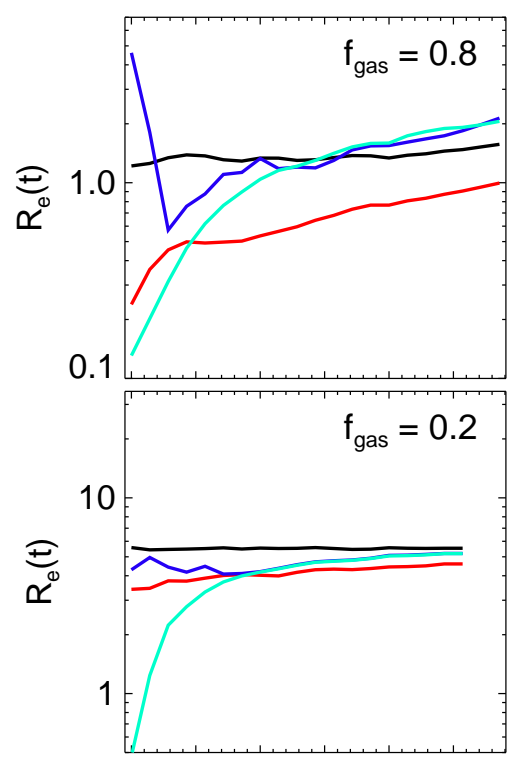

0.00 .51 .01 .52 .02 .5
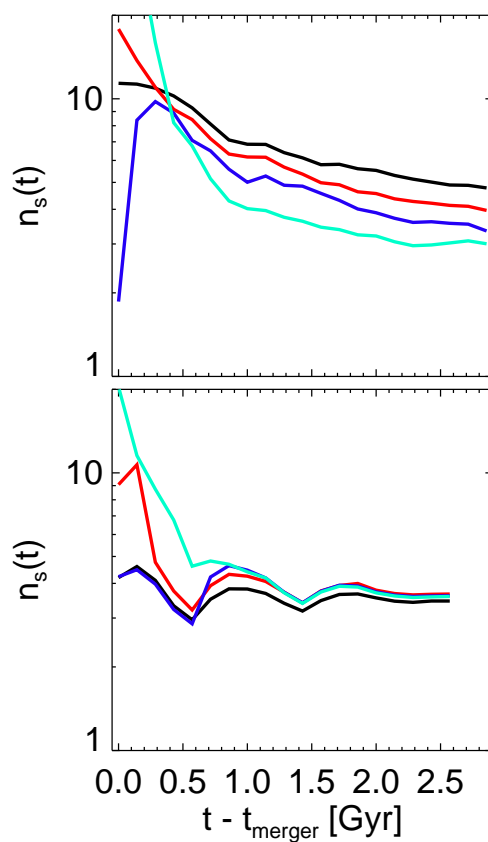

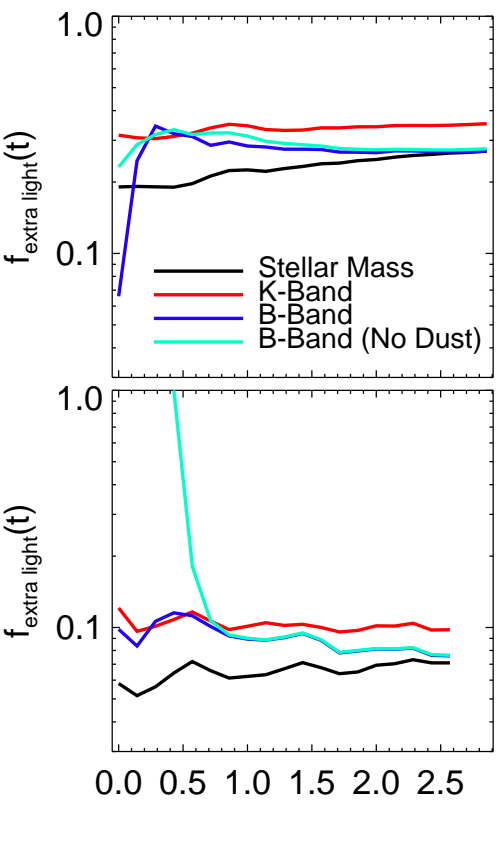

FIG. 20.- Evolution of surface brightness profile parameters as a function of time after the coalescence of merging nuclei, for a highly gas-rich major merger (top) and a less gas-rich major merger (bottom), in terms of the stellar mass profile, observed $K$-band (including stellar population age and metallicity effects and dust obscuration), observed $B$-band, and $B$-band ignoring dust obscuration (as labeled). Values shown at each time are medians across $\sim 100$ sightlines, the sightline-to-sightline variance is generally slightly smaller than the offsets in the plotted curves. Left: Half-light radius (directly from the light profiles). Middle: Outer profile Sersic index $n_{s}$. Right: Extra light fraction. The evolution in $n_{s}$ is largely real (and does not generally follow systematic patterns), and owes to relaxation of shells and other features; other evolution owes to stellar populations and dust.

Hernquist \& Quinn (1987) and Hernquist \& Spergel (1992). The oscillations occur as the material in shells moves in and out to large radii. As more orbits are completed, the shell stars phase-wrap, smoothing their projected density distribution, and so the ringing in $n_{s}$ damps out. These behaviors are not unusual, but there is considerable variation in the effects on $n_{s}$ with time - it is difficult to define a typical behavior for the relaxation of $n_{s}$. It is also clear that the degree to which $n_{s}$ might be different after the system is relaxed to that measured in the recent merger remnant stage is highly variable - there is no well-defined systematic bias (or even a typical direction of such a bias, to higher or lower $n_{s}$ ) in $n_{s}$ at early times versus late times. More likely, we expect that there will be less scatter in $n_{s}$ in the merger remnant sample of RJ04 once the objects have all relaxed (see also Naab \& Trujillo 2006).

The $K$-band profile reflects these trends - there is some (generally weak, even in the extreme high $f_{\text {gas }}$ case) time evolution owing to the weak dependence of $K$-band $M / L$ on stellar age. The sense of this is what is generally expected - since stars in the center of the remnant are formed in the final starburst and younger, they have larger $L / M$ at early times, and more of the light appears to come from inner radii, biasing $R_{e}$ to slightly smaller values. The effect is generally applicable for only $\sim$ a couple $\times 10^{8}$ yr after the merger, however, considerably shorter than the likely ages of most of the objects in the observed sample (excepting, perhaps, those still in the ULIRG/LIRG class). After this time, the age difference is small relative to the absolute ages of the populations, and makes little difference to $M / L$ as a function of radius.

That is not to say, however, that the $K$-band is free of bias. The measured quantities reflect the stellar mass profile with little additional time dependence, but are systematically offset by a small amount. This is because both of the examples shown have strong metallicity gradients, with higher metallicities in their central regions. In $K$-band, $L / M$ rises with metallicity, so this bias goes in the same direction as the age bias, but is more permanent. The metallicity gradient does not fade in the same manner as an initial age gradient. There is therefore a systematic bias towards underestimating $R_{e}$ and overestimating $f_{\text {extralight }}$ by roughly $\sim 20 \%$. The magnitude of this bias, however, is directly sensitive to the metallicity gradient, and observed ellipticals tend to have gradients ranging from nil to comparable strength to those above (discussed in detail in Hopkins et al. (2008b)), so the bias will range from object to object in this range. It is therefore difficult to systematically correct our analysis of the observed systems - but even if we uniformly apply the most extreme correction for this fact, it makes no difference to our conclusions, and the offsets implied are considerably smaller than the object-toobject scatter in these parameters. In fact, considering an ensemble of simulations, one is far more likely to introduce bias in $R_{e}$ and $f_{\text {extra light }}$ by not including sufficient dynamic range in the surface brightness profile used for fitting.

In $B$-band, the comparison is roughly as expected. If one ignores dust, there is an extremely strong time dependence of all the fitted parameters until $\sim 1$ Gyr after the merger. Dust, however, negates much of this, because the youngest stellar populations introducing the greatest bias also tend to be the most obscured. To the extent that this is typical, the biggest effects in $B$-band are also in the first $\sim$ couple $\times 10^{8} \mathrm{yr}$, where stellar populations are young and substantial dust exists which can make the best-fit parameters highly variable and sensitive to viewing angle. Interestingly, at late times after the merger, the optical light profile reflects the stellar mass profile with less bias than the NIR profile. This is because, in $B$ and other optical bands, the trend of $L / M$ with metallicity is opposite that with age: $L / M$ decreases with metallicity. Therefore the effects of age gradients and metallicity gradients actually tend to cancel one another out at late times. This suggests that, at early times after a merger (typical of the systems in the RJ04 
sample studied here) the NIR is a better tracer of the surface brightness profile, considerably less sensitive to age effects and dust-induced sightline-to-sightline variation. Once the systems are fully relaxed, however (provided there has been no new infall of gas and dust or new star formation event), optical light becomes similarly robust, and can actually trace the mass distribution with slightly less systematic bias. In practice, however, even evolved ellipticals often have dust lanes or central dust concentrations, so care must be taken regarding their effects.

\section{DISCUSSION}

We have studied the origin and properties of extra light or cusps in mergers of gas-rich galaxies using a large suite of both numerical simulations and local observed merger remnants. We confirm the original prediction of Mihos \& Hernquist (1994a) with our improved numerical models: namely, that tidal torques in major mergers of gasrich disks channel gas into the central regions of the galaxy, where it forms a dense central starburst. The starburst leaves a central light excess with a high phase space density, making the remnant more compact, reconciling the physical and phase space densities of disks and elliptical galaxies, and imprinting radial gradients into the remnant (Mihos \& Hernquist 1994b).

Stars in the remnant can be separated into three distinct populations. First, those stars which form in the progenitor disks before the final merger and coalescence of the two galaxies. The final merger scatters the orbits of these stars as they experience violent relaxation. They dominate the light, even in extremely gas-rich merger remnants, outside of $\sim 0.5-1 \mathrm{kpc}$, and develop a Sersic-law $n_{s} \sim 2.6 \pm 0.7$ profile owing to their partial violent relaxation. These stars are effectively the dissipationless component of the merger, and behave to lowest order much as they would entirely in the absence of gas. For example, the effective radius of just these stars reflects that of the progenitor disks (it is substantially larger than that of a typical elliptical of the same mass).

Second is the starburst population, formed in the central gas concentration during the final stages of the merger. Since dissipation allows the gas to lose energy rapidly, this component is very compact, and dominates the light inside a small radius $\lesssim 0.5-1 \mathrm{kpc}$, comparable to the observed scales of central starbursts in merging LIRGs and ULIRGs. These stars $d o$ not undergo violent relaxation - because the dynamical times in the center of the galaxy are short, the final coalescence has largely completed in these central regions by the time the starburst nears maximum. In other words, these stars form in a compact, dissipational starburst in a nearly fixed background potential set by the dissipationless component of the merger. We show that the size of this dissipational component is set primarily by the radius at which it becomes self-gravitating. The gas is then generally stable against further collapse (even with cooling) and rapidly forms stars. Since the star formation time scale and subsequent collapse are both regulated by the dynamical time (and the system is self-gravitating), the central component systematically processes its gas as it contracts. It's worth noting that merger-induced starbursts may not be the only source of dissipation (for example, stellar mass loss may replenish the gas supply and lead to new dissipational bursts, see e.g. Ciotti \& Ostriker 2007), but for our purposes, all dissipational star formation will appear similar when observed and have the same effects (we are essentially measuring the integrated amount of dissipation).
Third, some gas survives the merger. This is primarily material which has been moved to large radii temporarily, either blown out by a combination of supernova and AGN feedback or thrown out in tidal tails. This gas will then slowly settle back in, against the background of a largely relaxed remnant. Since there are no strong tidal torques remaining, the material quickly settles into a small, rotationally supported disk, and typically forms embedded kinematic components (embedded disks, kinematically decoupled cores, etc.; see Hernquist \& Barnes 1991; Hoffman et al. 2007). While potentially important for the kinematics of the remnant (rotation and isophotal shapes, in particular), this component rarely contributes substantially to the surface density profile of most objects, and therefore is not of immediate interest. Furthermore, this component has little effect on the key predictions here, regarding e.g. the degree of central dissipation and central mass concentration. Some caution should be taken, though, since in optical bands a younger embedded disk can appear much more prominent (for example, up to $\sim 20 \%$ of the $B$-band light, despite being only $\sim 2 \%$ of the stellar mass).

In principle, observed merger remnant light profiles can be decomposed into a central concentration tracing the dissipational starburst and an outer Sersic profile reflecting the dissipationless, violently relaxed stars. However, we show that any attempt to infer the extra light content of a galaxy requires care. Fitting the light profile of a typical, albeit very gas-rich merger remnant to a pure Sersic law or a cored Sersic law, for example, and comparing the central regions with the extrapolation of the outer profile yields physically meaningless values of both the outer Sersic index and the extra light fraction (in the cored Sersic case, the fit actually is biased towards inferring that these gas-rich merger remnants have missing light in their centers, i.e. some kind of central mass deficit). The values do not at all reflect the physical values: i.e. the amount of mass involved in the dissipational starburst or the profile of the violently relaxed (non-starburst) stars. That is not to say, however, that these are uniformly poor fits to the profiles - if one wishes to use such profiles to recover e.g. the effective radius or total light content of the galaxy, the bias is not severe (but see Boylan-Kolchin et al.2005). However, it highlights the fact that any parameterized fit will have degeneracies and can recover systematically different values even for a nominally similar parameter (such as the outer Sersic index).

By using our simulations as a testing ground, we can calibrate such a fit in order to design a parametric decomposition of the profile that recovers the physical values of interest. We find that a simple means of doing so is to fit the surface brightness profiles to the sum of an inner exponential (Sersic $n=1$ ) and outer Sersic (free $n$ ) profile. The choice of $n=1$ for the inner regions is not meant to say that this reflects the true shape of the central extra light extrapolated inwards to very small radii, which can be complex (with e.g. stellar clusters and features at $\ll 50 \mathrm{pc}$ Lauer et al. 2005). In fact, this fitting procedure should not be used or extrapolated to within $\sim 30-50 \mathrm{pc}$, which neither our simulations nor the observations to which we compare typically resolve (indeed, in experiments with some well-resolved profiles, we find that including these small radii when fitting can lead to misleading results and a much higher rate of catastrophic failures of the fit owing to the presence of extremely small-scale features that are unimportant for the overall profile). The reason for the choice of $n=1$ for the inner component is that it, on average, yields the correct decomposition in both total mass and radius 
between the starburst and non-starburst stars, and because it minimizes the degeneracy with the outer Sersic index (fitting an inner $n=4$, for example, would introduce a large degeneracy with an outer profile that had a similar $n \sim 4$ profile).

We apply this decomposition to our simulations and to a large sample of gas-rich merger remnants observed by RJ04, ranging from ULIRGs to shell ellipticals. We also directly fit each of the observed profiles to a suite of simulations i.e. determine the simulation mass profile which most closely matches that observed. We find that, in all cases, we have simulations which provide good matches to the observed systems, to better than the typical point-to-point variance inherent in the simulation surface brightness profiles $(\Delta \mu \lesssim 0.1)$. We also find that the physical starburst components in these best-fitting simulations are closely related to those that we fit directly to the observed profiles, lending further support to our procedure for decomposing the profiles.

Given our analysis, we can then study how the properties of the two components scale. For the outer profiles, we find that their Sersic indices are remarkably constant as a function of stellar mass or any other properties. Indeed, there appears to be a typical Sersic index $\sim 2.5-3$ for gas-rich merger remnants, with a scatter of $\sim 0.7$ about this median. We emphasize that this outer Sersic index is only meaningful in the sense of reflecting those violently relaxed stellar populations recovered in our two component decomposition. Fitting the entire profile to a single Sersic index can yield a very different result, and can introduce systematic trends (if e.g. the typical extra light fraction or size changes with mass). Given our attempt to carefully separate these components, then, this should not be surprising: the dissipationless component is simply acting under the influence of gravity, and is therefore completely selfsimilar across scales (see also Naab \& Trujillo 2006). Nevertheless, we make a prediction that gas-rich merger remnants should have this approximately constant Sersic index distribution. Subsequent gas-poor remergers may have different Sersic indices, as might pseudobulges or other low-mass bulges (and together these may drive a systematic dependence of Sersic index on mass, owing to the cosmological dependence of formation history on mass); but this particular class of ellipticals should have roughly a fixed Sersic index distribution.

The properties of the extra light component also scale in a regular fashion. For a given initial simulation gas fraction, the extra light content of the remnant systematically decreases with mass. This is expected, since the star formation efficiency is higher in larger mass systems, so they consume more of whatever gas they have at earlier stages (e.g. on first passage), before the final merger. What the extra light mass reflects is the gas content available and channeled to the center during the final coalescence of the two nuclei. Comparing the estimated extra light masses from our two-component decomposition to this physical value, we find a good correlation. There is substantial scatter - for a given object, the inferred extra light fraction based on fitting the profile can be misleading (in the sense of not reflecting the true starburst mass fraction) by a factor $\sim$ a couple, but, on average, the appropriate value is recovered.

In a few observed systems, detailed stellar population studies have enabled estimates of how much mass was recently formed in a merger-induced starburst (as opposed to more extended star formation prior to the merger). For these cases, then, we can directly compare our estimated extra light component masses to the young stellar populations, and find that indeed they trace one another to within a factor $\sim 2-3$, com- parable to the expected scatter in both estimators. Again, this suggests that we can in fact infer physical decompositions from observed merger remnant profiles.

There are a number of interesting applications of this decomposition, beyond noting the importance and physical nature of extra light in recent gas-rich merger remnants. In Hopkins et al. (2008b) we apply this analysis to large samples of old, relaxed elliptical galaxies with central cusps, and consider the role of the extra light in shaping the global kinematic properties of the galaxies. But we show here that one can in fact use this light component to infer something physical about the formation of the galaxy. Furthermore, we demonstrate in detail how the different components relevant to the surface brightness profiles of gas-rich merger remnants form and evolve. We also note that, with this physical decomposition in place, we often infer extra light fractions/masses and radii which are much larger than previous estimates (i.e. that extra light is typically $\sim 1-5 \%$ of the galaxy light and becomes important only within $\sim 0.05 R_{e}$ ). This is because the extra light component, as it becomes larger, typically blends in more smoothly with the outer profile, and does not necessarily appear as a sharp departure from the outer light profile. We see this in both simulations and observations - but in almost every such case there is additional evidence that the transition to extra light is real, including changes in the stellar populations, ellipticity, and boxy or diskiness of the remnants at these radii.

We find that gas-rich merger remnants do have excess light properties similar to those in gas-rich merger simulations. Given a careful two-component decomposition of the surface brightness profile (as opposed to fitting the entire profile to a single Sersic index, for example), we identify a statistically significant extra light component in every gas rich merger remnant observed (and simulated), with mass fractions spanning a wide range $\sim 3-30 \%$. There are good simulation analogues to each observed merger remnant, and they similarly contain this range of masses involved in their final mergerinduced starbursts. These extra light masses correspond to reasonable, expected initial gas fractions for the merging disks (although there is no one-to-one correspondence). They are also comparable to the estimated fractions in Hernquist et al. (1993), namely the dissipational mass fraction needed to explain the discrepancies between the maximum phase space densities and surface brightness of ellipticals and their progenitor spiral galaxies. We therefore confirm in observations of gas-rich merger remnants the long-standing theoretical prediction that sufficient dissipation is required to explain this discrepancy in the central profiles of ellipticals.

We have studied these properties and identified robust trends across a large suite of simulations, in which we vary e.g. the galaxy masses, initial gas fractions, concentrations, halo masses, presence or absence of bulges, presence or absence of black holes, feedback parameters from supernovae and stellar winds, orbital parameters and disk inclinations, and mass ratios of the merging galaxies. This range of parameters allows us to identify the most important physics. Most of these choices, for example, affect the surface brightness profile, extra light mass and radius of the extra light, concentration and effective radius of the remnant, and even its ellipticity and isophotal shape only indirectly. Ultimately, what determines the structure of the remnant (insofar as the properties we have considered) is, to first order, how much mass is in the dissipationless (violently relaxed) component versus the dissipational/starburst component at the time of the 
final coalescence of the merging galaxies. Therefore, varying e.g. the orbital parameters or initial galaxy structure can alter the remnant substantially, but predominantly only insofar as it changes the amount of gas which will be available at the time of the final coalescence of the galaxy nuclei (i.e. how much mass ends up in the starburst component, as opposed to being violently relaxed in the merger).

In the simplest possible scenario outlined above, the central light and dissipationless component arise simultaneously in a single gas-rich merger. In practice, the situation need not be so simple. It is well-established that spheroids undergoing subsequent mergers will conserve rank order in particle (stellar) binding energy and (correspondingly) mass profile shape (Barnes 1992). As a consequence, we expect that remergers or "dry" mergers will conserve the central light excesses (starburst component) originally established in a gasrich merger, even if the entire galaxy profile expands by a typical factor $\sim 2$ as it doubles in mass. In Hopkins et al. (2008a), we explicitly confirm in numerical simulations that the distinction between the remnant excess light and dissipationless outer profile is conserved in successive re-mergers, and that our methodology continues to reliably separate the original dissipational (compact, merger-driven starburst) and dissipationless (disk) components. This applies just as well to bulges in early-type galaxies: regardless of their formation mechanism (whether formed "in situ" by disk instabilities or minor mergers, or formed by earlier major mergers with a reaccreted disk), they will fundamentally be composed of a dissipationless (scattered) component and a dissipational (starburst driven by energy and angular momentum loss in gas) component, and these components will be separately conserved in subsequent mergers. For any merger remnant or elliptical galaxy, therefore, the observed central or excess light component should most generally be thought of as the sum of all compact starburst components formed in the history of the galaxy, and the outer light likewise as the sum of dissipationlessly scattered disk stars. In other words, if a spiral is transformed to a progressively earlier type (and, eventually, a true elliptical) by, say, a rapid series of many $5: 1$ mergers, each of which disrupts some disk stars (scattering them into a spheroid) and causes some gas to lose angular momentum and produce a small nuclear starburst (building up the central light component), then the final inner and outer light components will reflect the sum of the dissipational and dissipationless events.

Our physical interpretation of the extra light, and our conclusions and comparisons in this work, are therefore not changed by the merger histories of observed galaxies, nor by e.g. whether or not the progenitor disks have bulges - it is simply possible that some of the central mass concentration was built up in multiple previous events along with the most recent merger (but the total gas/dissipational content involved is conserved). Of course, in modeling those progenitor bulges, this raises the important question of how their structure should be initialized: some choice must be adopted (just as if one were to construct an "initial" elliptical to model a "dry" merger) for the initial mass fraction in a very compact dissipational component (which will, then, remain compact or "extra" light in the final merger remnant) versus the more extended dissipationless component (which will become part of the dissipationless component of the final remnant). Fortunately, to the extent that (at least "classical") bulges obey the same parameter correlations as ellipticals, this suggests that we can estimate such properties and what they imply for the original progenitors and their gas content by restricting our study to bulge-dominated systems or pure ellipticals.

We note that there is considerable room for progress in modeling the extra light component itself - including its kinematics, the shape of the surface brightness profile at small radii ( $\ll 100 \mathrm{pc}$ ), and the structure of galactic nuclei near a central black hole. Observations are rapidly making progress in this area, and revealing new insights into the formation histories of elliptical galaxies. Unfortunately, modeling these radii in a meaningful sense will require fundamental improvements in numerical simulations. At present, our spatial resolution reaches $\sim 30 \mathrm{pc}$. In principle, it would not be hard to improve this to $\lesssim 10 \mathrm{pc}$. However, at these scales, we approach the sizes of structure in the ISM - i.e. individual giant molecular clouds, star forming regions, and massive star clusters or galactic stellar nuclei. Without simulations which can self-consistently form these structures (i.e. include the multiple gas phases of the ISM and their exchange), as well as resolve e.g. individual supernova blastwaves and remnants, improved spatial resolution has no physical meaning. It is therefore an important and ambitious goal that the next generation of studies of galactic nuclei move beyond the sub-resolution prescriptions necessary when modeling large scales and attempt to include star formation, supernova feedback, and realistic, resolved ISM structure in simulations of galaxy mergers.

In earlier work (e.g. Hopkins et al. 2006a b, 2008c d) we developed a model linking starbursts, quasar activity, the growth of supermassive black holes, and the origin of ellipticals through evolutionary phases of the same events, driven by mergers of gas-rich galaxies. There is much observational support for the various links in this chain. ULIRGs are invariably associated with gas-rich mergers (e.g. Sanders \& Mirabel 1996) and have bolometric properties similar to bright quasars (e.g. Sanders et al. 1988a b), suggesting that ULIRGs evolve into quasars. By the Soltan argument (Soltan 1982; Hopkins et al. 2007d), the bulk of the cosmic mass density in supermassive black holes was accumulated during periods of bright quasar activity. Observed correlations between supermassive black holes and properties of their host ellipticals (Magorrian et al. 1998; Ferrarese \& Merritt 2000; Gebhardt et al. 2000) demonstrate that they formed together, not independently. To the extent that gas-rich mergers were responsible for growing most of the mass in supermassive black holes, these correlations strongly endorse the view that ellipticals formed originally in gas-rich mergers of spirals. (With the possibility that they could have been modified subsequently in gas-free mergers with other ellipticals that did not trigger quasar activity or lead to black hole growth, and that modest black hole growth can occur with non-merger induced fueling (Hopkins \& Hernquist 2006).)

In addition to these observational lines of evidence, there are simple physical arguments that support these connections. The bulk of the stellar mass in ellipticals was likely assembled dissipationlessly through violent relaxation (Lynden-Bell 1967), but the growth of supermassive black holes probably involved accretion of gas (Lynden-Bell 1969). As we argue in e.g. Hopkins et al. (2008d), the condition that supermassive black holes and ellipticals originate together requires their assembly in gas-rich mergers, which contain a galaxy's worth supply of both gas and stars. Our analysis here lends additional credence to this hypothesis: the outer components of the light profiles of ellipticals were indeed put into place through self-similar gravitational physics, but the 
compact, inner starburst populations resulted from gas dissipation. The same gas dissipation that yielded this inner component also provided the material to grow supermassive black holes in ellipticals in a self-regulated manner, accounting for the similarities between the fundamental plane of ellipticals (Dressler et al.1987; Djorgovski \& Davis 1987) and the black hole fundamental plane (Hopkins et al. 2007a.b). Observationally, then, we see that this blending of dissipationless stellar dynamics and gas dissipation is reflected not only in correlations between supermassive black holes and their hosts, but in the detailed structure of elliptical galaxies as well.

We thank our referee, Thorsten Naab, for comments contributing to the content and presentation of this paper. This work was supported in part by NSF grants ACI 96-19019, AST 00-71019, AST 02-06299, and AST 03-07690, and NASA ATP grants NAG5-12140, NAG5-13292, and NAG513381. Support for TJC was provided by the W. M. Keck Foundation.

\section{REFERENCES}

Barnes, J. E. 1988, ApJ, 331, 699

-. 1992, ApJ, 393, 484

Barnes, J. E., \& Hernquist, L. 1996, ApJ, 471, 115

Barnes, J. E., \& Hernquist, L. E. 1991, ApJ, 370, L65

Bell, E. F., \& de Jong, R. S. 2000, MNRAS, 312, 497

-. 2001, ApJ, 550, 212

Bell, E. F., McIntosh, D. H., Katz, N., \& Weinberg, M. D. 2003, ApJS, 149, 289

Bender, R. 1988, A\&A, 193, L7

Bender, R., Doebereiner, S., \& Moellenhoff, C. 1987, A\&A, 177, L53

Bournaud, F., Jog, C. J., \& Combes, F. 2005, A\&A, 437, 69

Boylan-Kolchin, M., Ma, C.-P., \& Quataert, E. 2005, MNRAS, 362, 184

Bruzual, G., \& Charlot, S. 2003, MNRAS, 344, 1000

Bryant, P. M., \& Scoville, N. Z. 1999, AJ, 117, 2632

Bullock, J. S., Kolatt, T. S., Sigad, Y., Somerville, R. S., Kravtsov, A. V. Klypin, A. A., Primack, J. R., \& Dekel, A. 2001, MNRAS, 321, 559

Busha, M. T., Evrard, A. E., Adams, F. C., \& Wechsler, R. H. 2005 ,

MNRAS, 363, L11

Carlberg, R. G. 1986, ApJ, 310, 593

Chabrier, G. 2003, PASP, 115, 763

Ciotti, L., \& Ostriker, J. P. 2007, ApJ, 665, 1038

Côté, P., et al. 2006, ApJS, 165, 57

Cote, P., et al. 2007, ApJ, 671, 1456

Cox, T. J., Di Matteo, T., Hernquist, L., Hopkins, P. F., Robertson, B., \& Springel, V. 2006a, ApJ, 643, 692

Cox, T. J., Dutta, S. N., Di Matteo, T., Hernquist, L., Hopkins, P. F., Robertson, B., \& Springel, V. 2006b, ApJ, 650, 791

Dasyra, K. M., et al. 2006, ApJ, 638, 745

-. 2007, ApJ, 657, 102

Davé, R., Hernquist, L., Katz, N., \& Weinberg, D. H. 1999, ApJ, 511, 521

de Vaucouleurs, G. 1948, Annales d'Astrophysique, 11, 247

Dekel, A., \& Cox, T. J. 2006, MNRAS, 370, 1445

Di Matteo, T., Springel, V., \& Hernquist, L. 2005, Nature, 433, 604

Djorgovski, S., \& Davis, M. 1987, ApJ, 313, 59

Downes, D., \& Solomon, P. M. 1998, ApJ, 507, 615

Doyon, R., Wells, M., Wright, G. S., Joseph, R. D., Nadeau, D., \& James, P. A. 1994, ApJ, 437, L23

Dressler, A., Lynden-Bell, D., Burstein, D., Davies, R. L., Faber, S. M., Terlevich, R., \& Wegner, G. 1987, ApJ, 313, 42

Erb, D. K., Steidel, C. C., Shapley, A. E., Pettini, M., Reddy, N. A., \& Adelberger, K. L. 2006, ApJ, 646, 107

Faber, S. M., Tremaine, S., Ajhar, E. A., Byun, Y.-I., Dressler, A., Gebhardt, K., Grillmair, C., Kormendy, J., Lauer, T. R., \& Richstone, D. 1997, AJ, 114,1771

Ferrarese, L., \& Merritt, D. 2000, ApJ, 539, L9

Ferrarese, L., et al. 2006, ApJS, 164, 334

Flores, H., Hammer, F., Puech, M., Amram, P., \& Balkowski, C. 2006, A\&A, 455, 107

Gebhardt, K., et al. 2000, ApJ, 539, L13

Genzel, R., Tacconi, L. J., Rigopoulou, D., Lutz, D., \& Tecza, M. 2001, ApJ, 563,527

Goto, T. 2005, MNRAS, 357, 937

Graham, A. W. 2001, AJ, 121, 820

Graham, A. W., Erwin, P., Trujillo, I., \& Asensio Ramos, A. 2003, AJ, 125, 2951

Hernquist, L. 1989, Nature, 340, 687

-. 1990, ApJ, 356, 359

-. 1992, ApJ, 400, 460

-. 1993a, ApJ, 404, 717

-. 1993b, ApJ, 409, 548

Hernquist, L., \& Barnes, J. E. 1991, Nature, 354, 210

Hernquist, L., \& Mihos, J. C. 1995, ApJ, 448, 41
Hernquist, L., \& Quinn, P. J. 1987, ApJ, 312, 1

Hernquist, L., \& Spergel, D. N. 1992, ApJ, 399, L117

Hernquist, L., Spergel, D. N., \& Heyl, J. S. 1993, ApJ, 416, 415

Hibbard, J. E., \& Yun, M. S. 1999, ApJ, 522, L93

Hoffman, L., et al. 2007, ApJ, in preparation

Hopkins, P. F., Cox, T. J., Dutta, S. N., Hernquist, L., Kormendy, J., \&

Lauer, T. R. 2008a, ApJ, submitted [astro-ph]

—. 2008b, ApJ, submitted [astro-ph]

Hopkins, P. F., Cox, T. J., Kereš, D., \& Hernquist, L. 2008c, ApJS, 175, 390

Hopkins, P. F., \& Hernquist, L. 2006, ApJS, 166, 1

Hopkins, P. F., Hernquist, L., Cox, T. J., Di Matteo, T., Martini, P.,

Robertson, B., \& Springel, V. 2005a, ApJ, 630, 705

Hopkins, P. F., Hernquist, L., Cox, T. J., Di Matteo, T., Robertson, B., \& Springel, V. 2006a, ApJS, 163, 1

Hopkins, P. F., Hernquist, L., Cox, T. J., \& Kereš, D. 2008d, ApJS, 175, 356

Hopkins, P. F., Hernquist, L., Cox, T. J., Robertson, B., \& Krause, E. 2007a, ApJ, 669, 45

-. 2007b, ApJ, 669, 67

Hopkins, P. F., Hernquist, L., Cox, T. J., Robertson, B., \& Springel, V. 2006b, ApJS, 163, 50

Hopkins, P. F., Hernquist, L., Martini, P., Cox, T. J., Robertson, B., Di

Matteo, T., \& Springel, V. 2005b, ApJ, 625, L71

Hopkins, P. F., Lidz, A., Hernquist, L., Coil, A. L., Myers, A. D., Cox, T. J., \& Spergel, D. N. 2007c, ApJ, 662, 110

Hopkins, P. F., Richards, G. T., \& Hernquist, L. 2007d, ApJ, 654, 731

James, P., Bate, C., Wells, M., Wright, G., \& Doyon, R. 1999, MNRAS, 309,585

Joseph, R. D., \& Wright, G. S. 1985, MNRAS, 214, 87

Katz, N., Weinberg, D. H., \& Hernquist, L. 1996, ApJS, 105, 19

Kennicutt, Jr., R. C. 1998, ApJ, 498, 541

Kormendy, J. 1999, in Astronomical Society of the Pacific Conference Series, Vol. 182, Galaxy Dynamics - A Rutgers Symposium, ed. D. R.

Merritt, M. Valluri, \& J. A. Sellwood, 124-+

Kormendy, J., Fisher, D. B., Cornell, M. E., \& Bender, R. 2007, ApJ, submitted

Laine, S., van der Marel, R. P., Lauer, T. R., Postman, M., O’Dea, C. P., \& Owen, F. N. 2003, AJ, 125, 478

Lake, G. 1989, AJ, 97, 1312

Lake, G., \& Dressler, A. 1986, ApJ, 310, 605

Lauer, T. R., et al. 2005, AJ, 129, 2138

-. 2007, ApJ, 664, 226

Lynden-Bell, D. 1967, MNRAS, 136, 101

-. 1969, Nature, 223, 690

Magorrian, J., et al. 1998, AJ, 115, 2285

McKee, C. F., \& Ostriker, J. P. 1977, ApJ, 218, 148

Michard, R. 2006, A\&A, 449, 519

Mihos, J. C., \& Hernquist, L. 1994a, ApJ, 437, L47

-. 1994b, ApJ, 427, 112

-. 1994c, ApJ, 431, L9

-. 1996, ApJ, 464, 641

Milosavljević, M., Merritt, D., Rest, A., \& van den Bosch, F. C. 2002, MNRAS, 331, L51

Naab, T., Jesseit, R., \& Burkert, A. 2006, MNRAS, 372, 839

Naab, T., \& Ostriker, J. P. 2007, MNRAS, in press [astro-ph/0702535]

Naab, T., \& Trujillo, I. 2006, MNRAS, 369, 625

Navarro, J. F., Frenk, C. S., \& White, S. D. M. 1996, ApJ, 462, 563

O’Shea, B. W., Nagamine, K., Springel, V., Hernquist, L., \& Norman, M. L.

2005, ApJS, 160, 1

Ostriker, J. P. 1980, Comments on Astrophysics, 8, 177

Prugniel, P., \& Simien, F. 1997, A\&A, 321, 111

Quillen, A. C., Bower, G. A., \& Stritzinger, M. 2000, ApJS, 128, 85

Quinn, P. J. 1984, ApJ, 279, 596 
Ravindranath, S., Ho, L. C., Peng, C. Y., Filippenko, A. V., \& Sargent, W. L. W. 2001, AJ, 122, 653

Reichardt, C., Jimenez, R., \& Heavens, A. F. 2001, MNRAS, 327, 849

Rest, A., van den Bosch, F. C., Jaffe, W., Tran, H., Tsvetanov, Z., Ford, H. C., Davies, J., \& Schafer, J. 2001, AJ, 121, 2431

Robertson, B., Bullock, J. S., Cox, T. J., Di Matteo, T., Hernquist, L., Springel, V., \& Yoshida, N. 2006a, ApJ, 645, 986

Robertson, B., Cox, T. J., Hernquist, L., Franx, M., Hopkins, P. F., Martini, P., \& Springel, V. 2006b, ApJ, 641, 21

Robertson, B., Hernquist, L., Cox, T. J., Di Matteo, T., Hopkins, P. F., Martini, P., \& Springel, V. 2006c, ApJ, 641, 90

Rothberg, B., \& Joseph, R. D. 2004, AJ, 128, 2098

-. 2006a, AJ, 131, 185

-. 2006b, AJ, 132, 976

Sanders, D. B., \& Mirabel, I. F. 1996, ARA\&A, 34, 749

Sanders, D. B., Soifer, B. T., Elias, J. H., Madore, B. F., Matthews, K., Neugebauer, G., \& Scoville, N. Z. 1988a, ApJ, 325, 74

Sanders, D. B., Soifer, B. T., Elias, J. H., Neugebauer, G., \& Matthews, K. 1988b, ApJ, 328, L35

Schweizer, F. 1996, AJ, 111, 109

Schweizer, F. 1998, in Saas-Fee Advanced Course 26: Galaxies: Interactions and Induced Star Formation, ed. R. C. Kennicutt, Jr., F. Schweizer, J. E. Barnes, D. Friedli, L. Martinet, \& D. Pfenniger, 105-+

Schweizer, F., \& Seitzer, P. 1992, AJ, 104, 1039

-. 1998, AJ, 116, 2206

-. 2007, AJ, 133, 2132
Shapley, A. E., Coil, A. L., Ma, C.-P., \& Bundy, K. 2005, ApJ, 635, 1006

Shier, L. M., \& Fischer, J. 1998, ApJ, 497, 163

Solomon, P. M., Downes, D., Radford, S. J. E., \& Barrett, J. W. 1997, ApJ, 478,144

Soltan, A. 1982, MNRAS, 200, 115

Springel, V. 2005, MNRAS, 364, 1105

Springel, V., Di Matteo, T., \& Hernquist, L. 2005a, ApJ, 620, L79

-. 2005b, MNRAS, 361, 776

Springel, V., \& Hernquist, L. 2002, MNRAS, 333, 649

-. 2003, MNRAS, 339, 289

-. 2005, ApJ, 622, L9

Tacconi, L. J., Genzel, R., Lutz, D., Rigopoulou, D., Baker, A. J., Iserlohe, C., \& Tecza, M. 2002, ApJ, 580, 73

Titus, T. N., Spillar, E. J., \& Johnson, P. 1997, AJ, 114, 958

Toomre, A. 1977, in Evolution of Galaxies and Stellar Populations, ed. B. M. Tinsley \& R. B. Larson, 401

Toomre, A., \& Toomre, J. 1972, ApJ, 178, 623

Trujillo, I., et al. 2006, ApJ, 650, 18

Vitvitska, M., Klypin, A. A., Kravtsov, A. V., Wechsler, R. H., Primack, J. R., \& Bullock, J. S. 2002, ApJ, 581, 799

Woods, D. F., Geller, M. J., \& Barton, E. J. 2006, AJ, 132, 197

Younger, J., et al. 2007a, ApJ, in preparation

Younger, J. D., Cox, T. J., Seth, A. C., \& Hernquist, L. 2007b, ArXiv e-prints, 707

APPENDIX

FITS TO THE SAMPLE OF RJ04

In Figures 21,38 we explicitly show the results of our fitting and simulation comparison with each of the merger remnants in the sample of RJ04. Table 1 summarizes the results, including the estimated integrated properties from simulations corresponding to each observed system. 
Extra Central Light in Merger Remnants
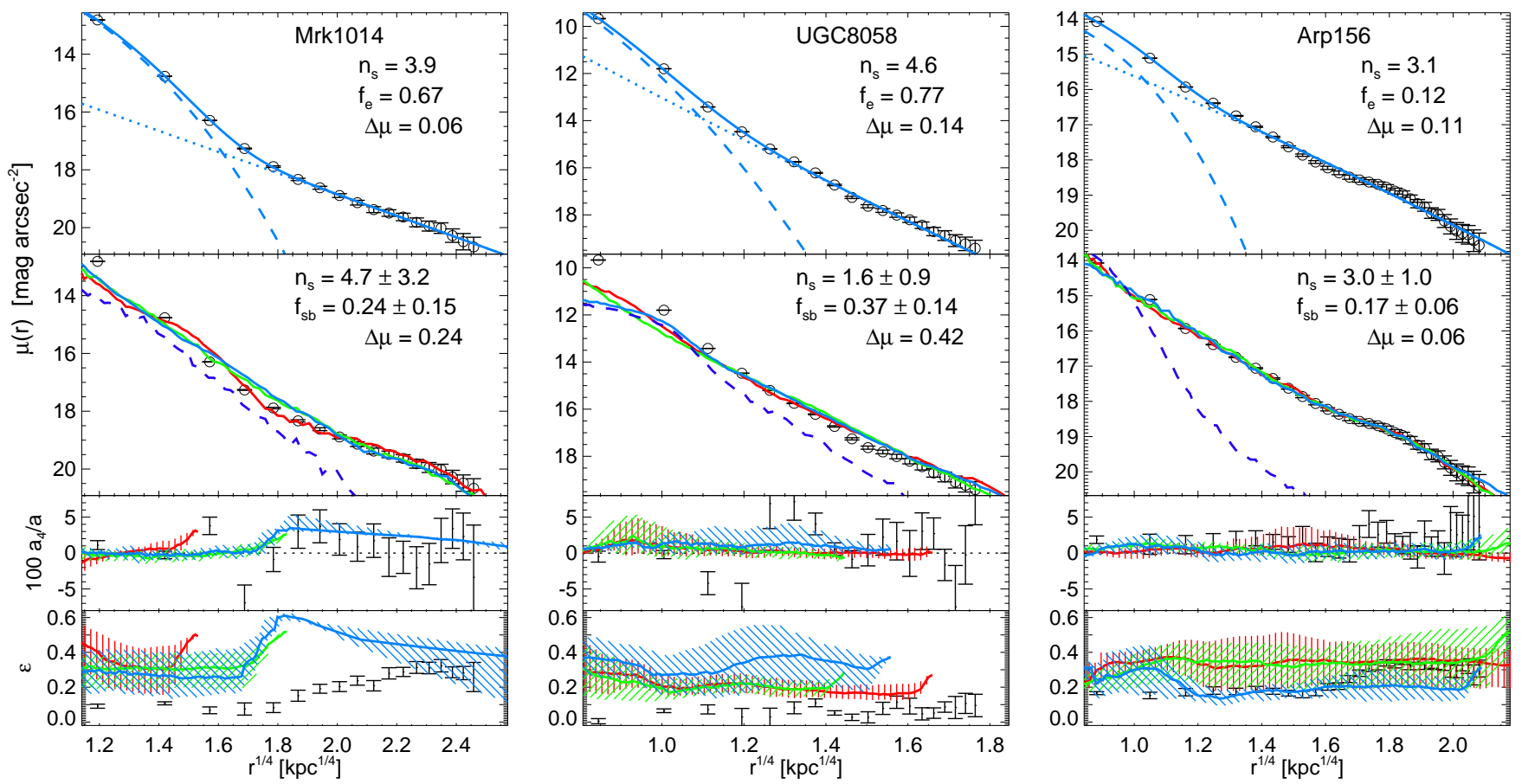

FIG. 21.- As Figure6 Figures 21 38 show the results for all objects in the RJ04 sample. Objects are sorted from most to least luminous in $K$-band. Note that Mrk1014 (previously unpublished) and UGC8058 (Mrk231) shown here are contaminated by central AGN, giving rise to the large discrepancies and poor fits seen.
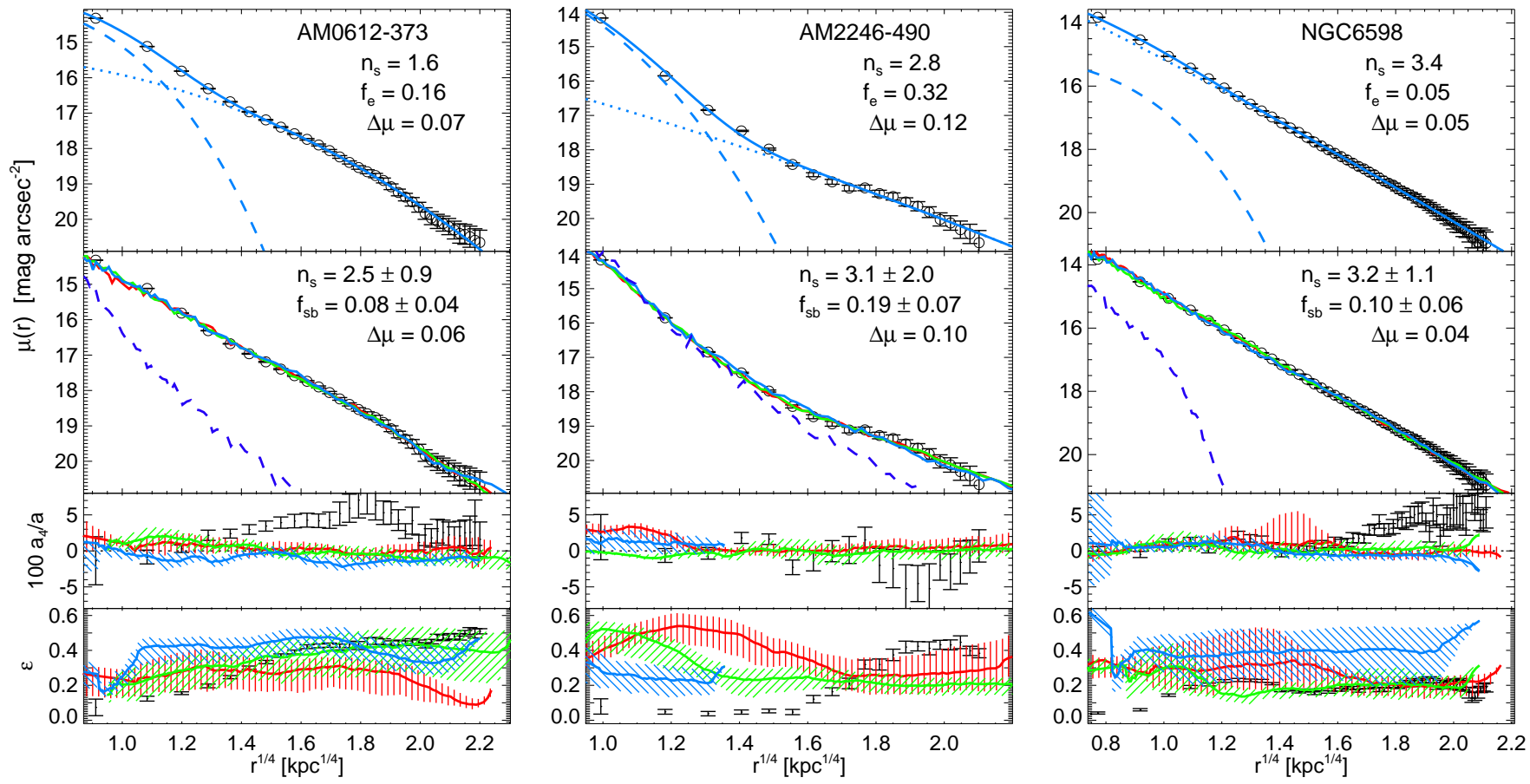

FIG. 22.- Figure21 continued. 
Hopkins et al.
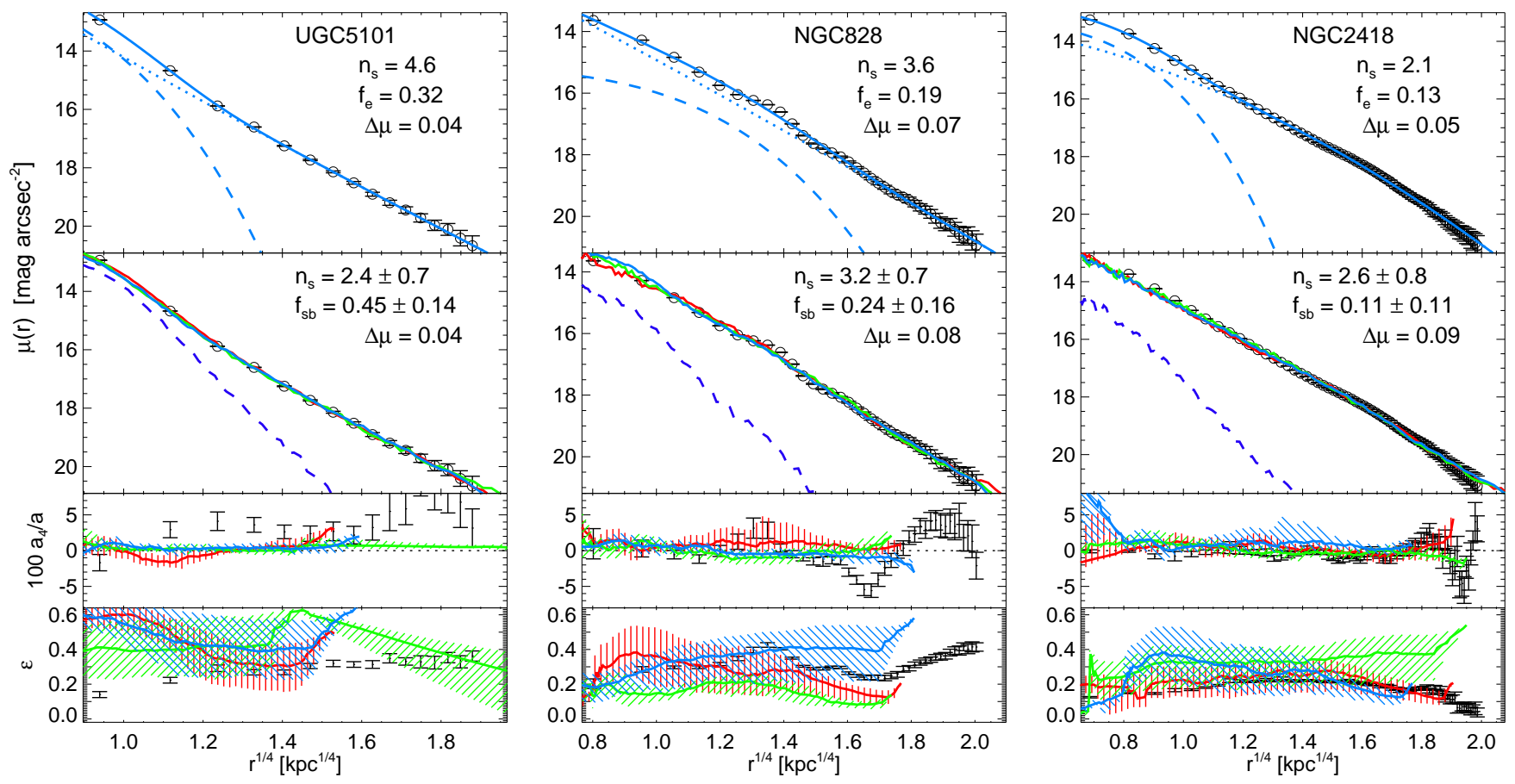

FIG. 23.- Figure 21 continued.
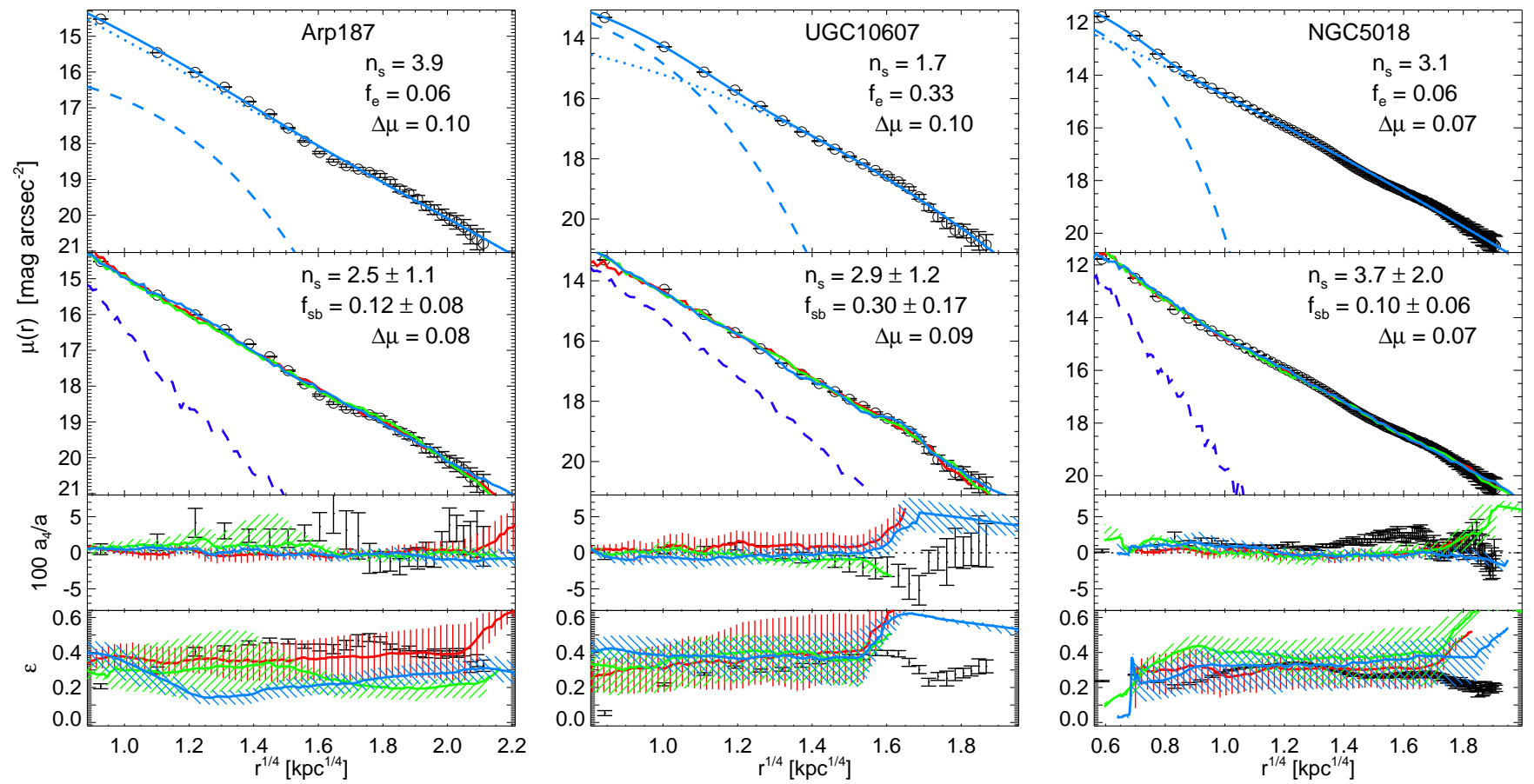

FIG. 24.- Figure 21 continued. 
Extra Central Light in Merger Remnants
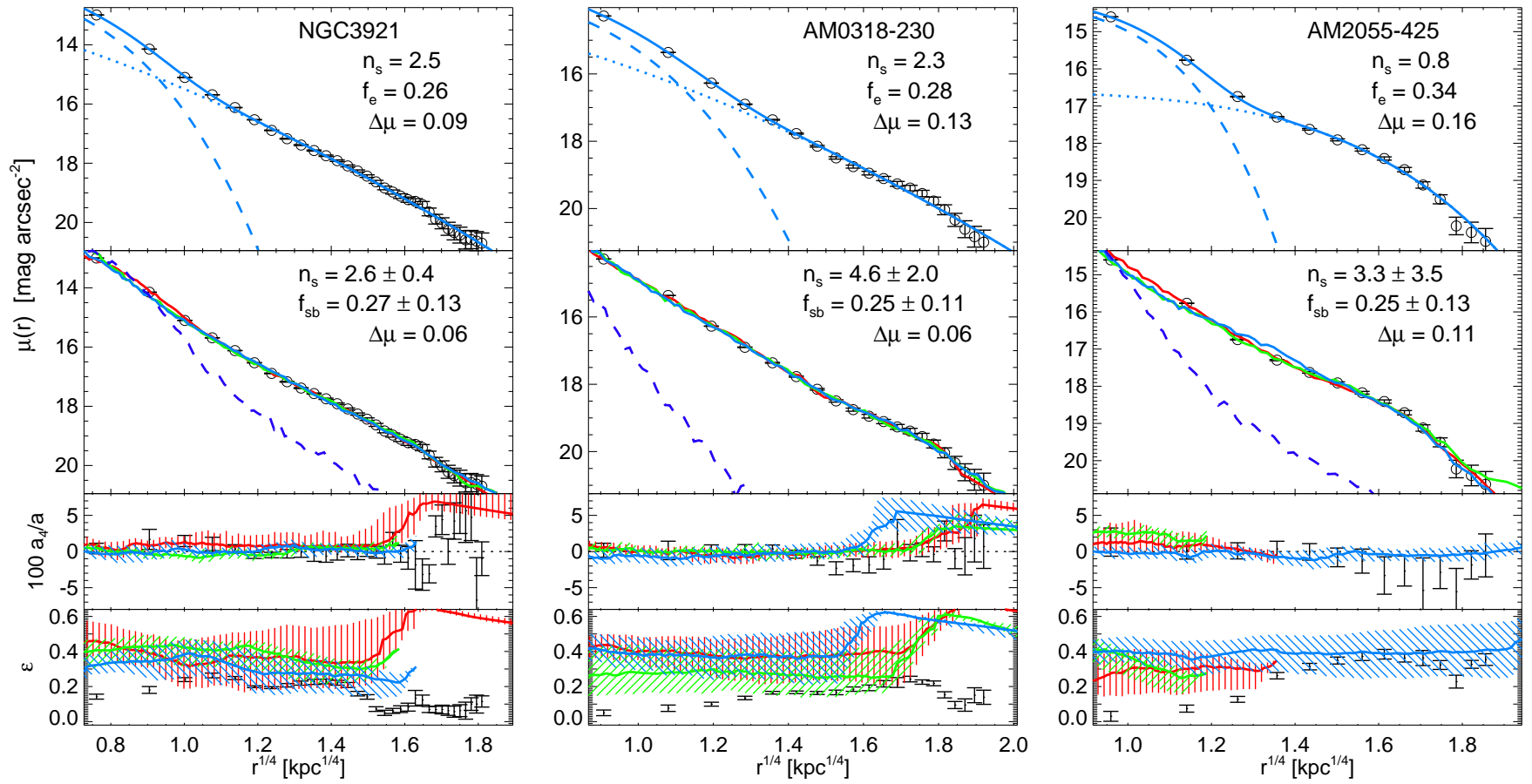

FIG. 25.- Figure 21 continued.
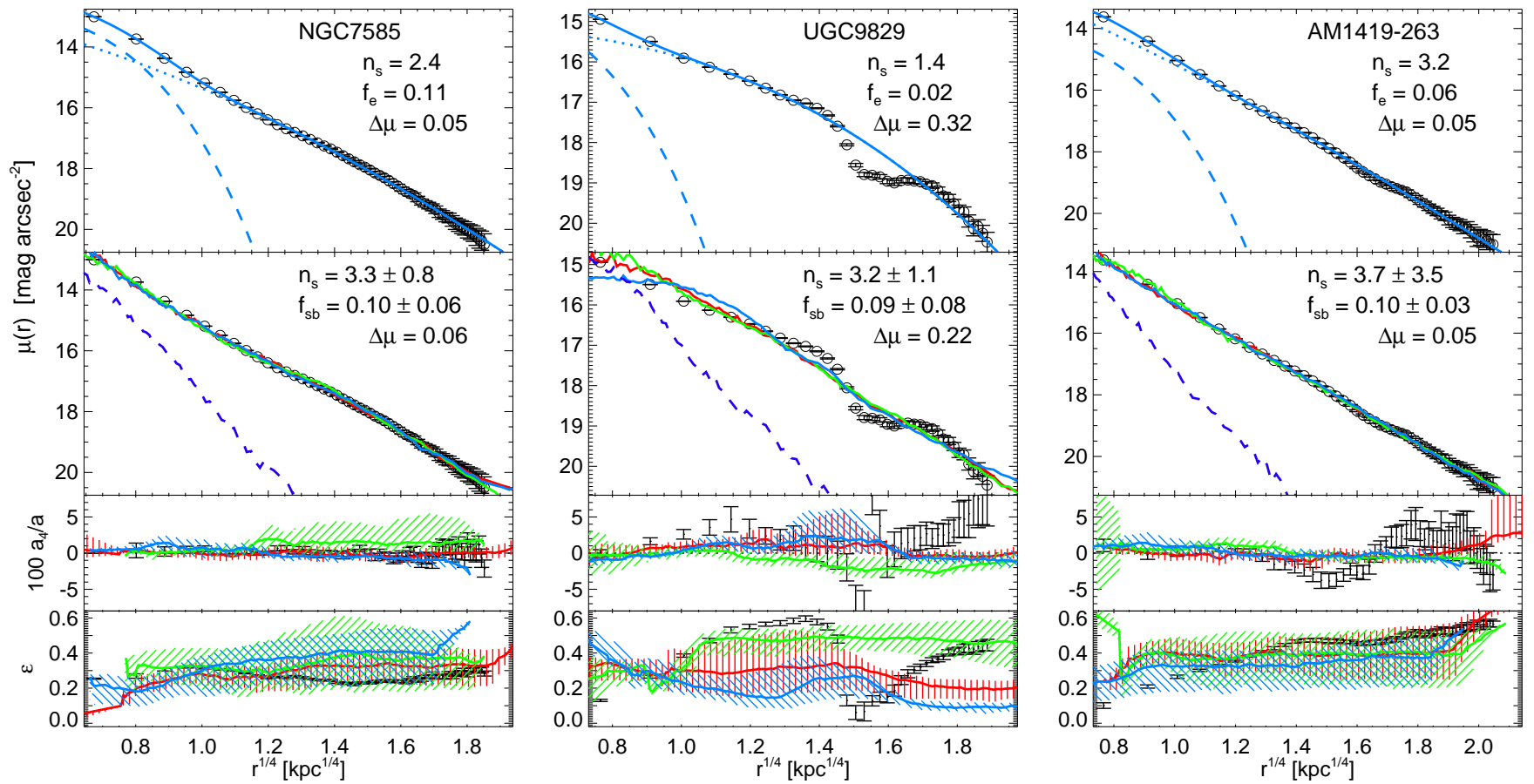

FIG. 26.- Figure21 continued. Unrelaxed features (including remaining spiral and tidal structure) in UGC9829 prevent a reliable fit. 

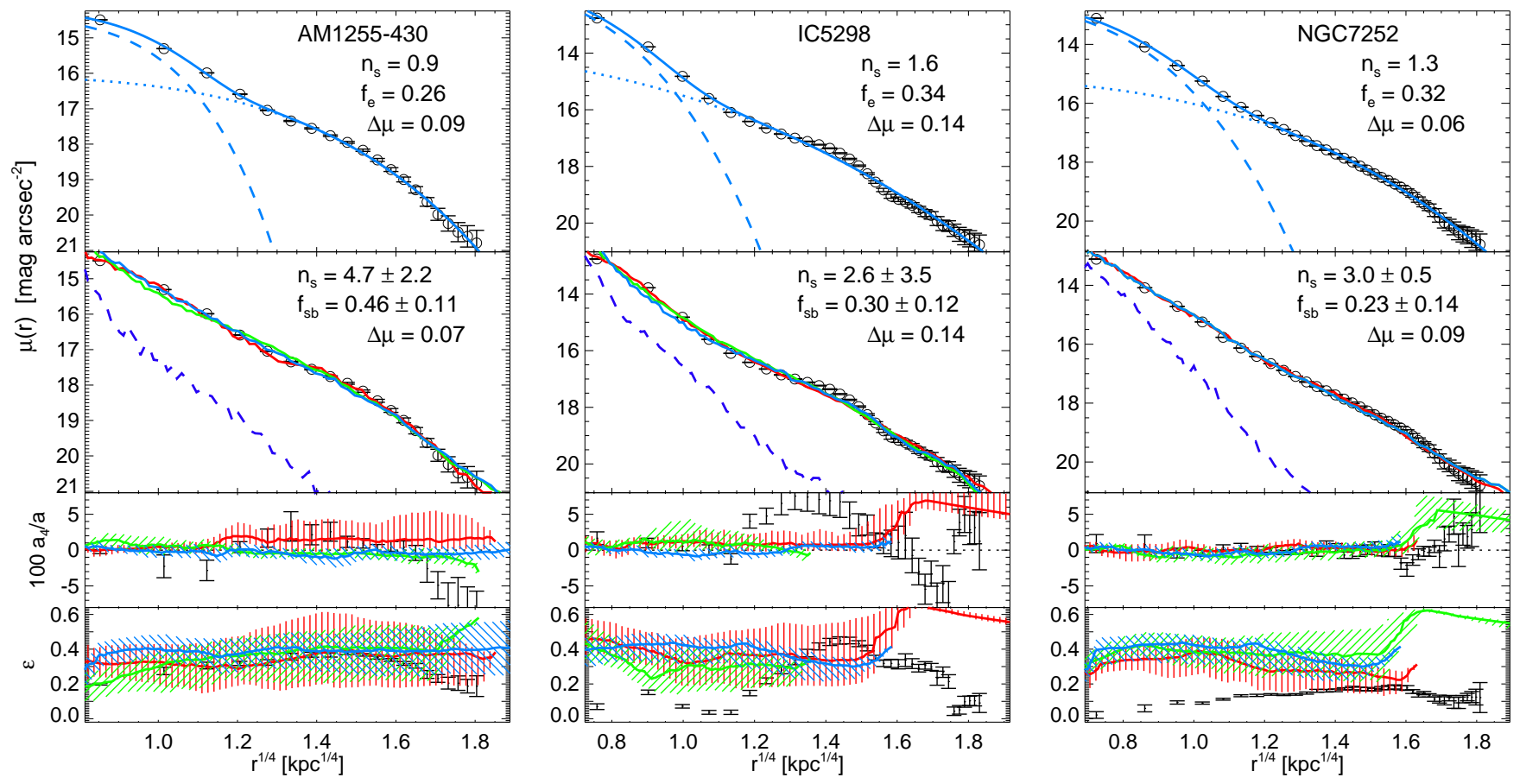

FIG. 27.- Figure 21 continued.
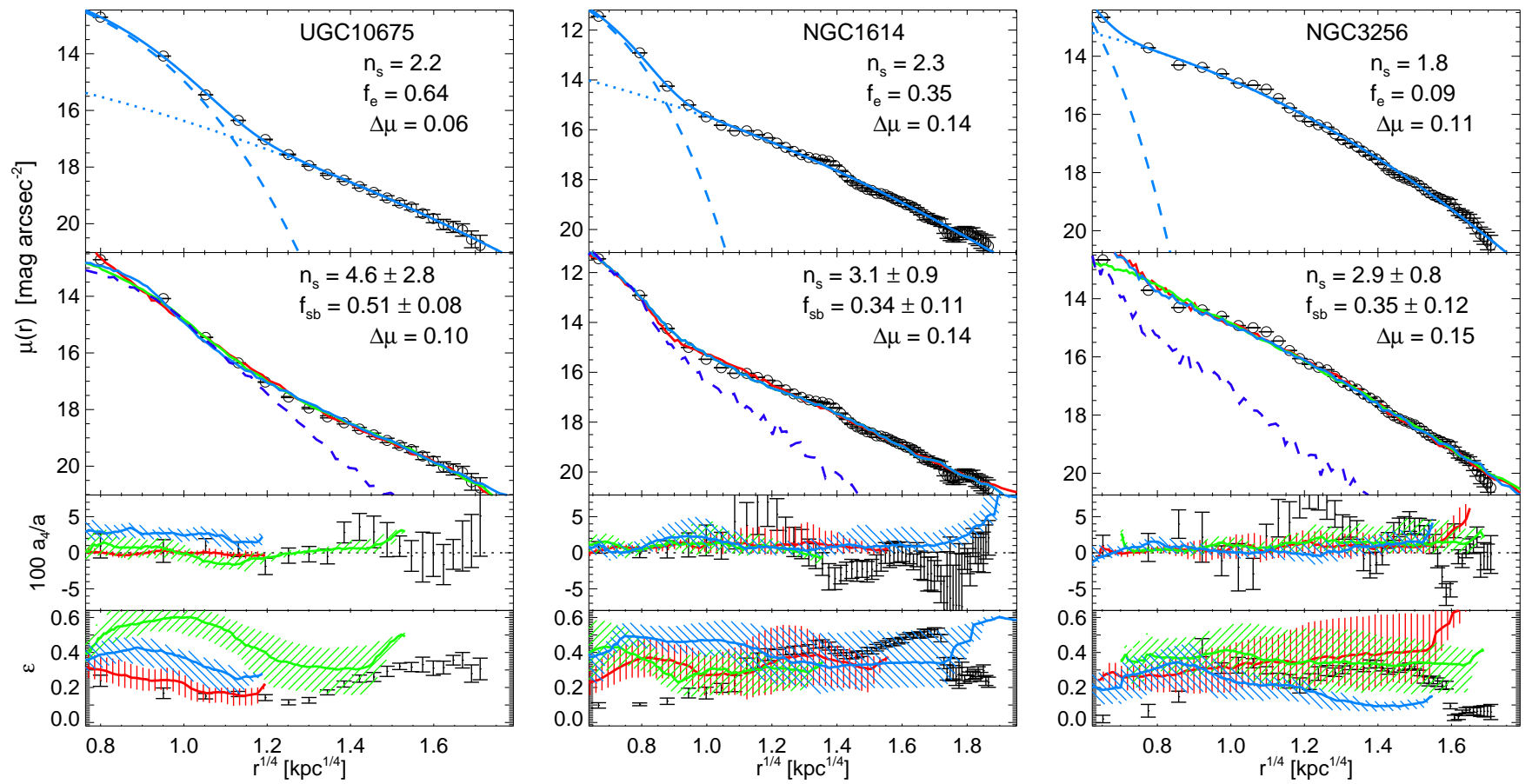

FIG. 28.- Figure 21 continued. 
Extra Central Light in Merger Remnants
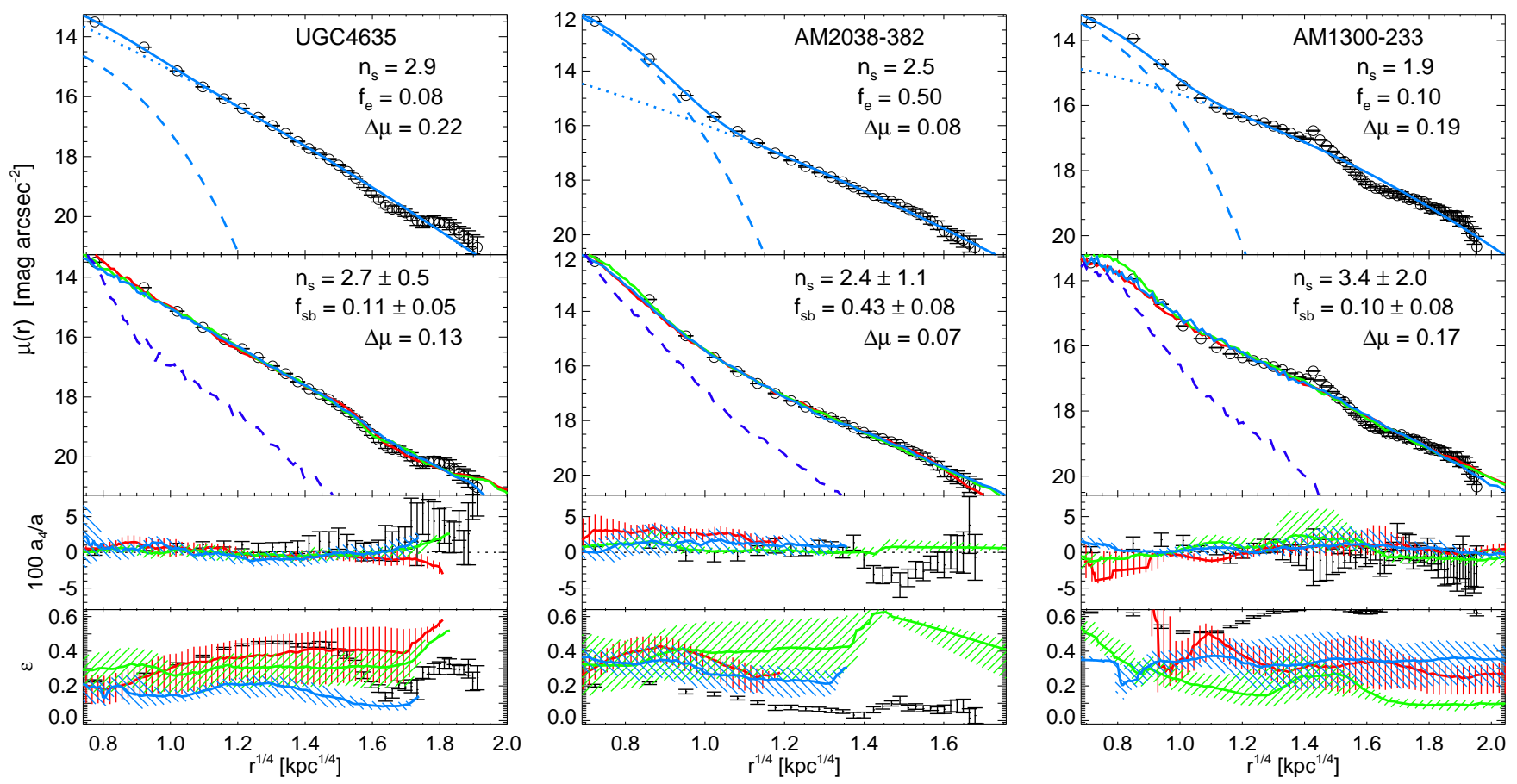

FIG. 29.- Figure 21 continued.
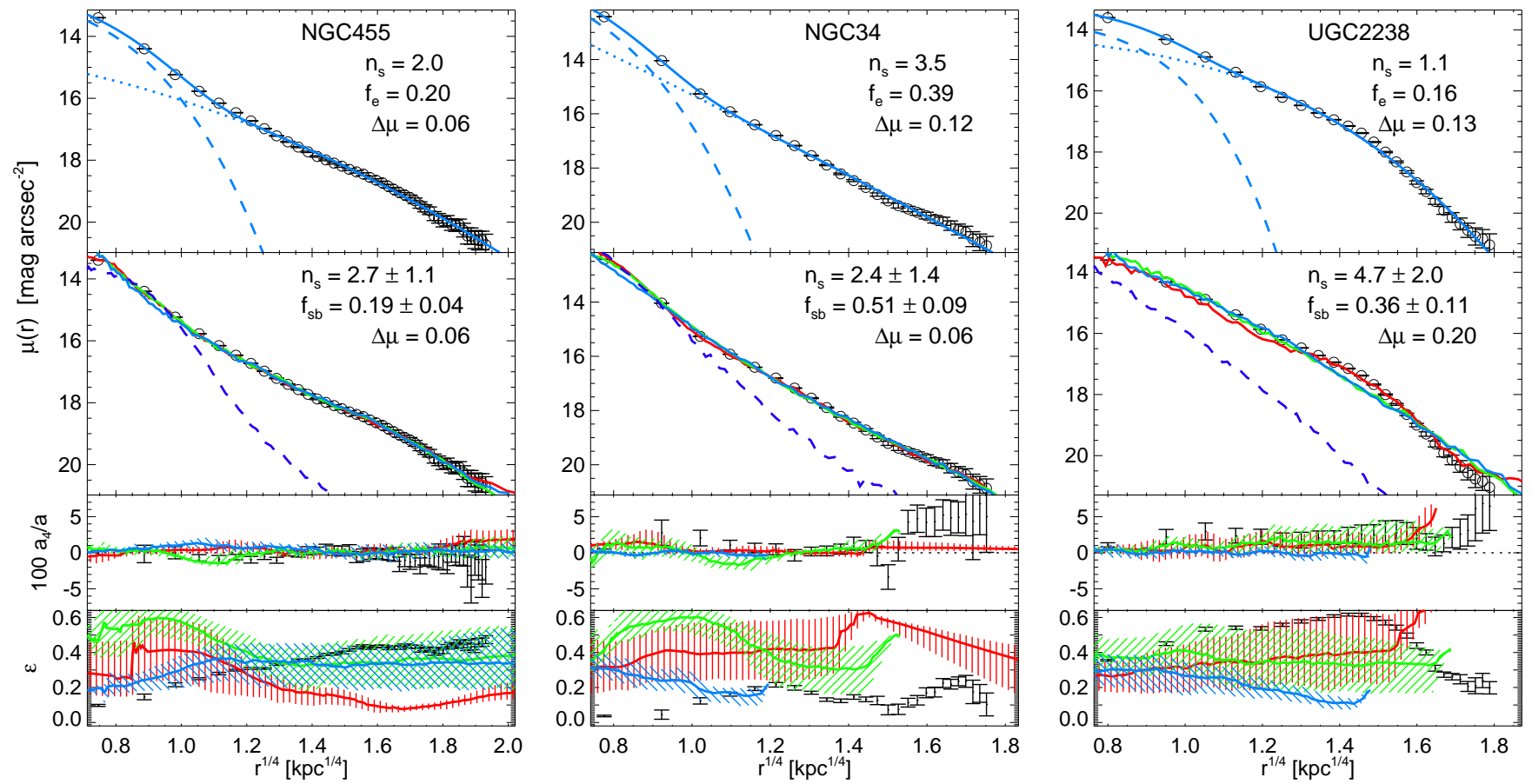

FIG. 30.- Figure 21 continued. UGC2238 contains a prominent edge-on central disk. 

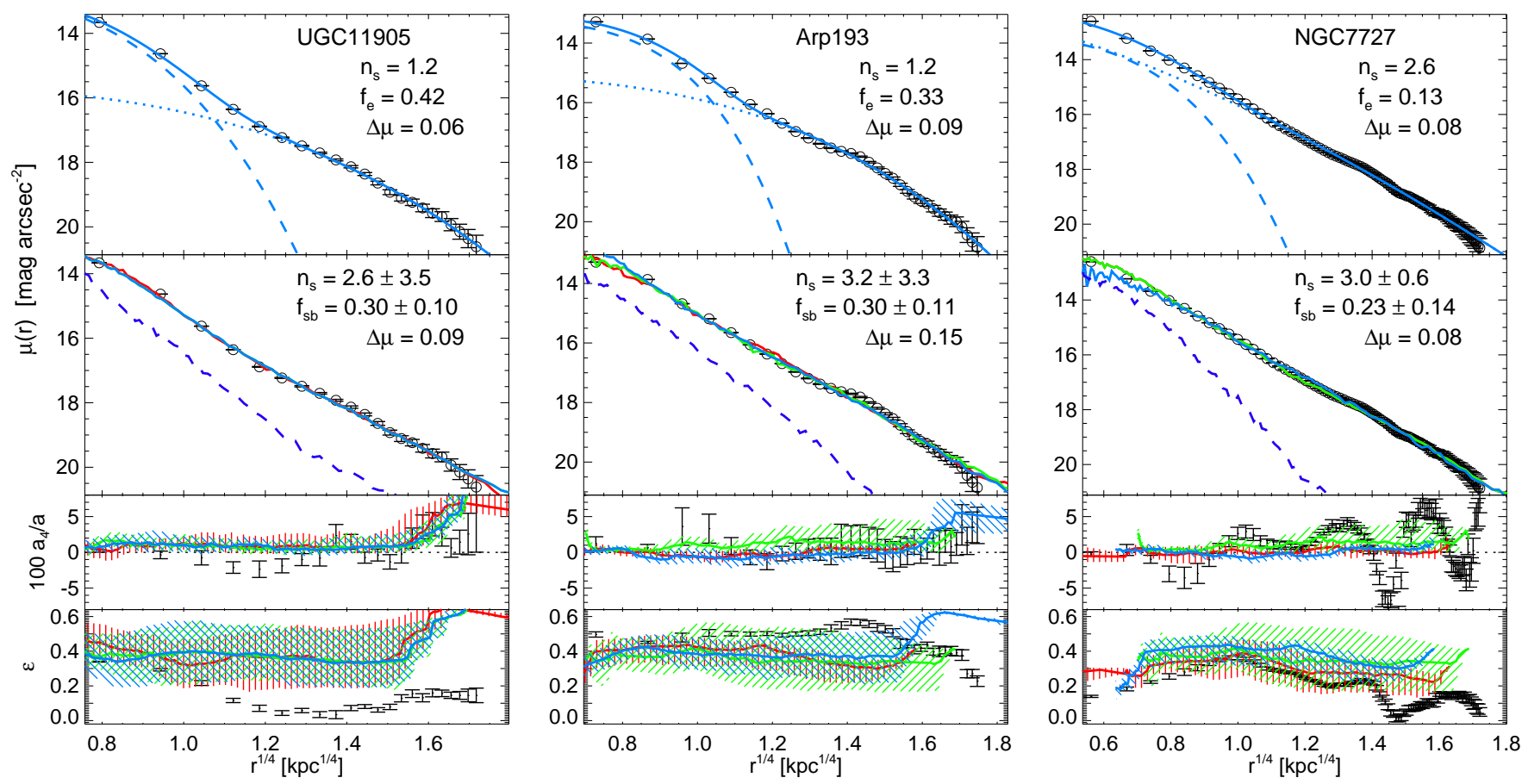

FIG. 31.-Figure 21 continued.
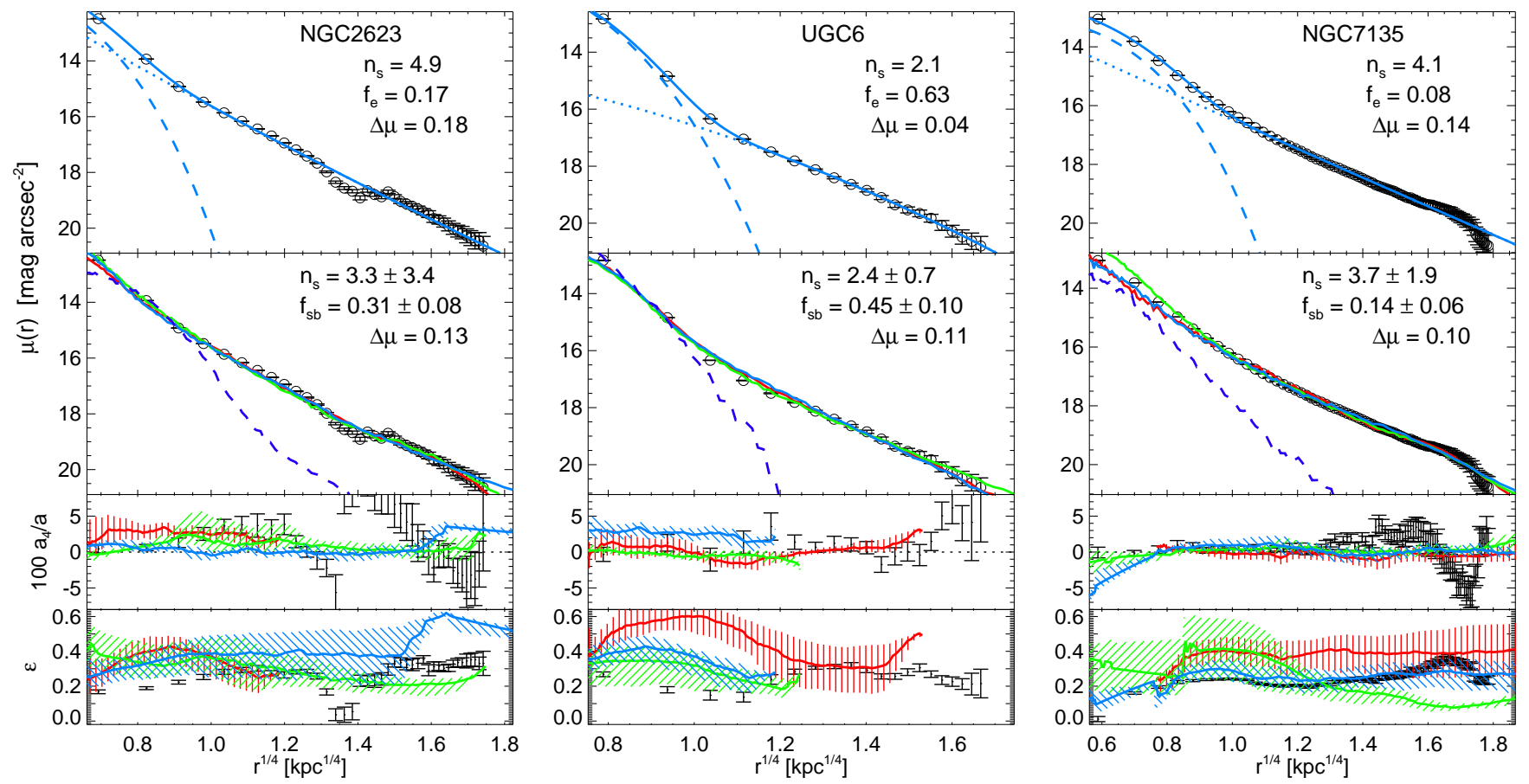

FIG. 32.- Figure 21 continued. 
Extra Central Light in Merger Remnants
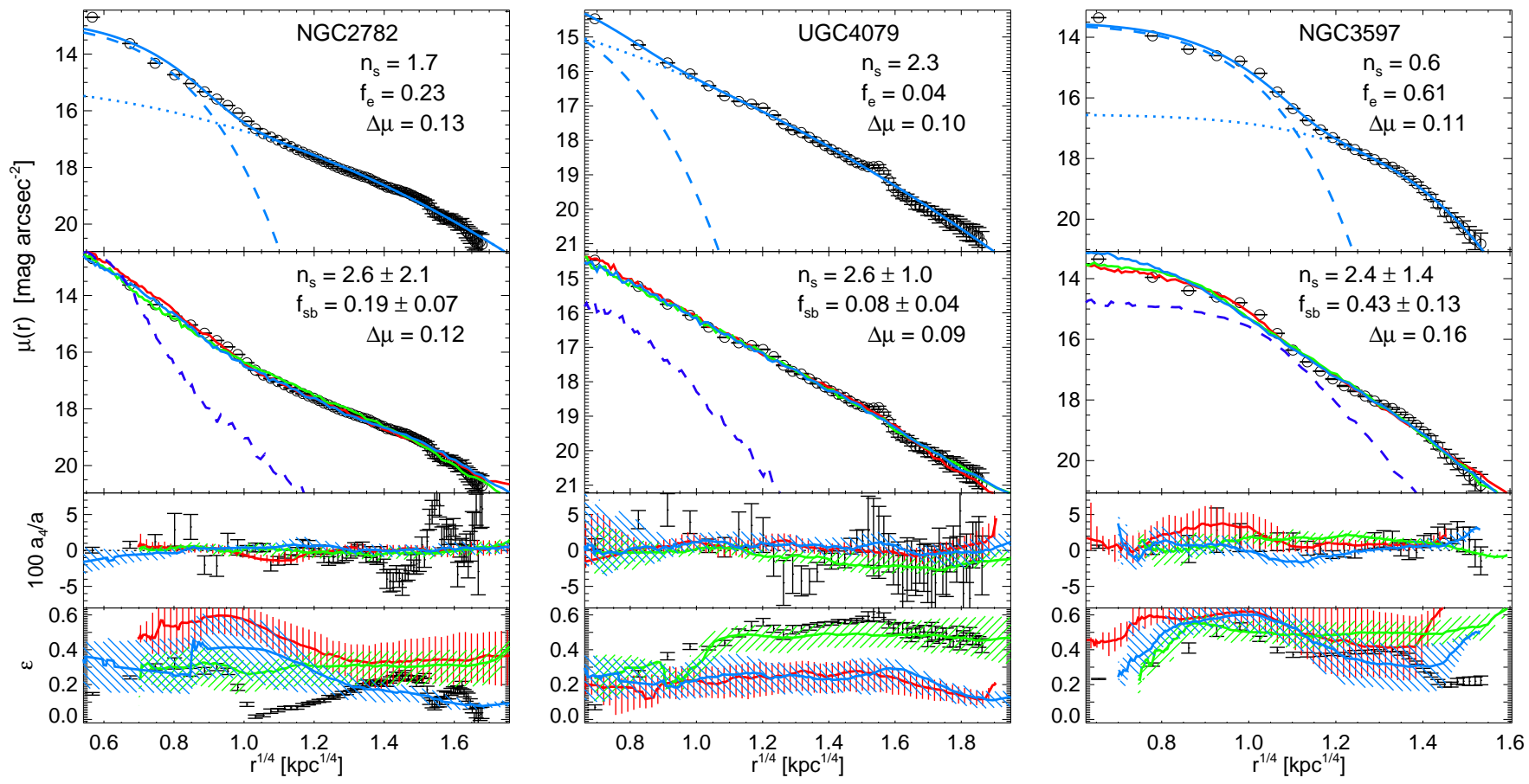

FIG. 33.- Figure21 continued.
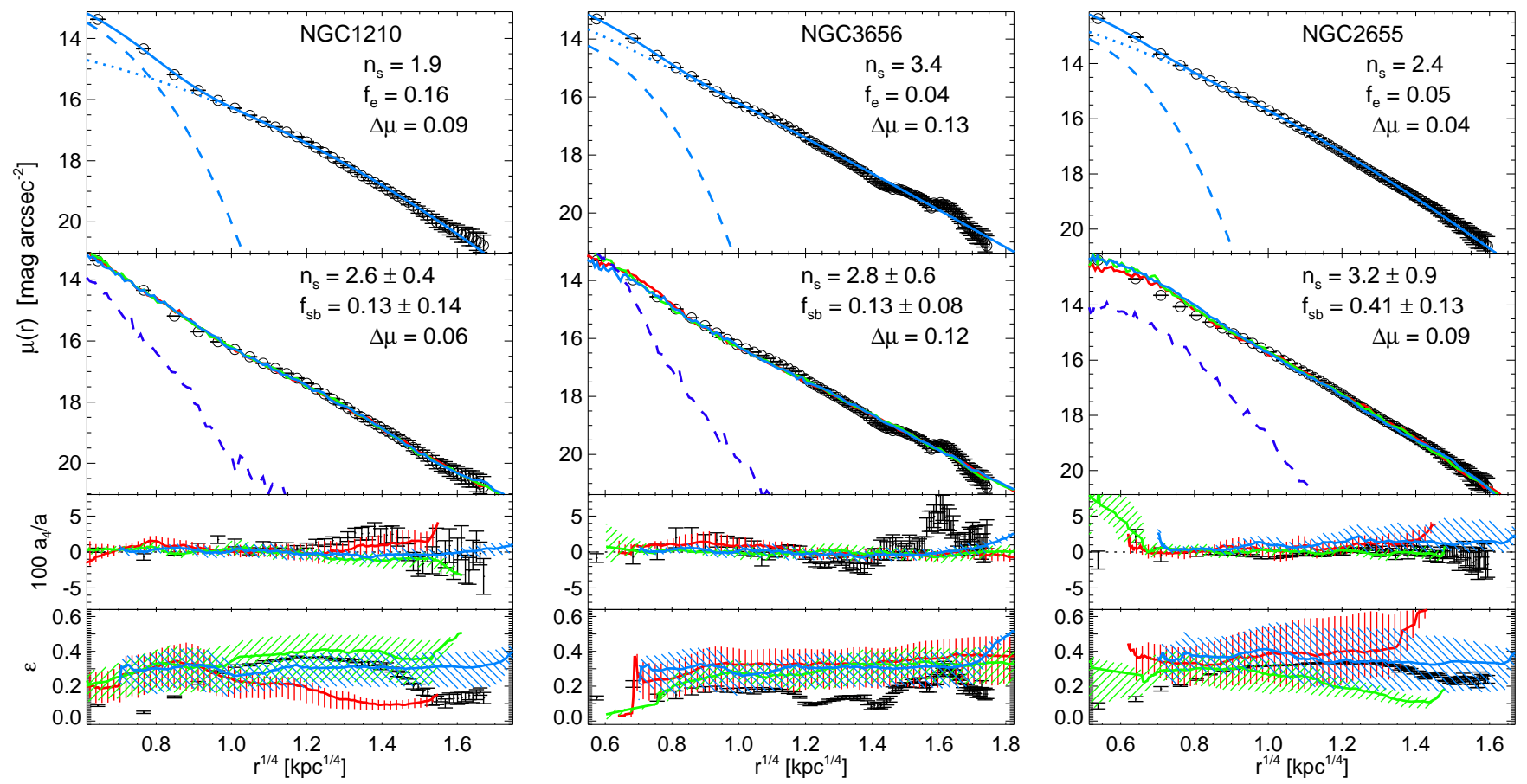

FIG. 34.- Figure 21 continued. 

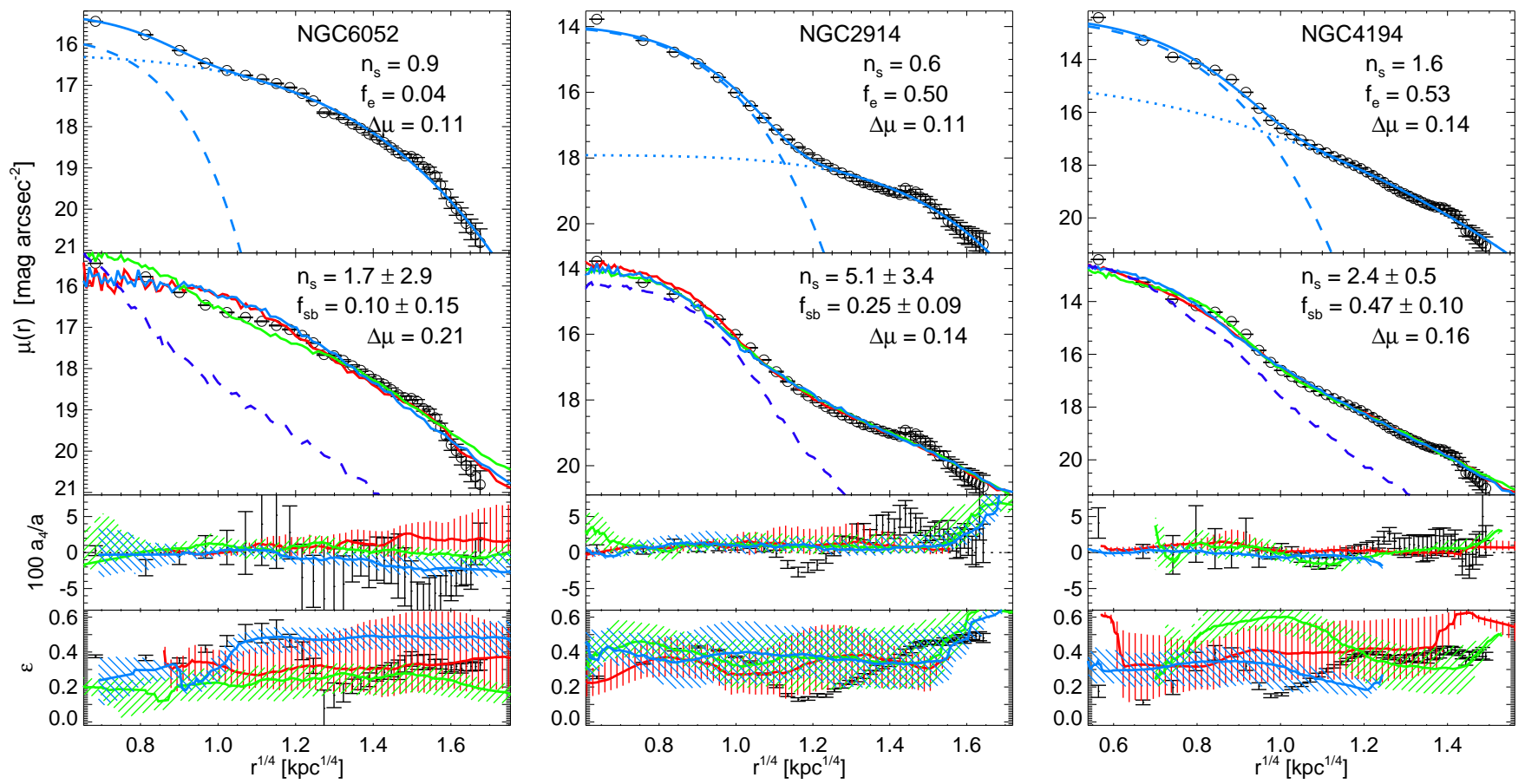

FIG. 35.- Figure 21 continued. NGC6052 appears to be still unrelaxed at $\sim$ a few kpc.
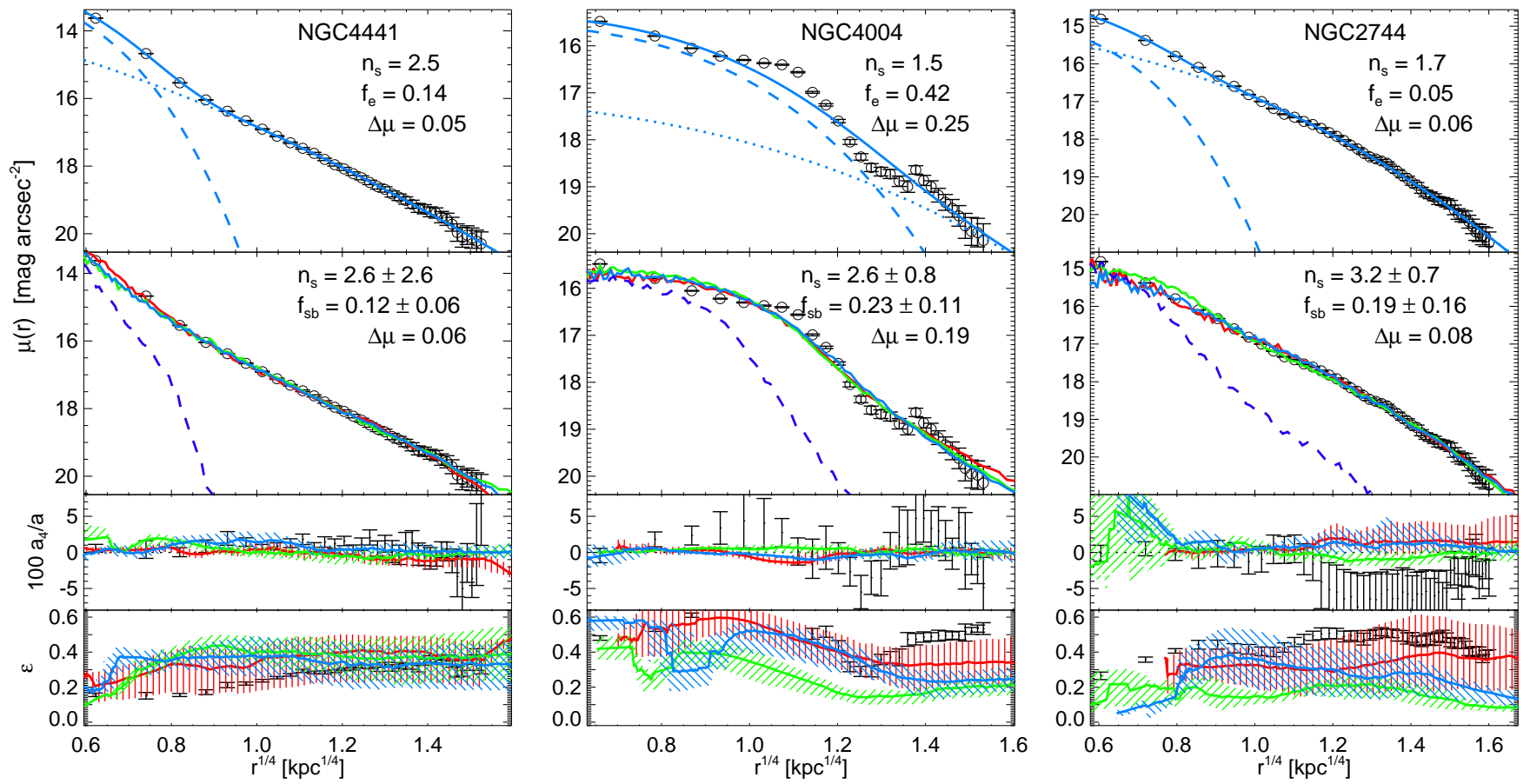

FIG. 36.- Figure 21 continued. There is a thin, bar-like feature at $\sim 1-2 \mathrm{kpc}$ in NGC4004, not reproduced in our simulations at the observed snapshots (although similar features do generically occur in earlier merger stages). 
Extra Central Light in Merger Remnants
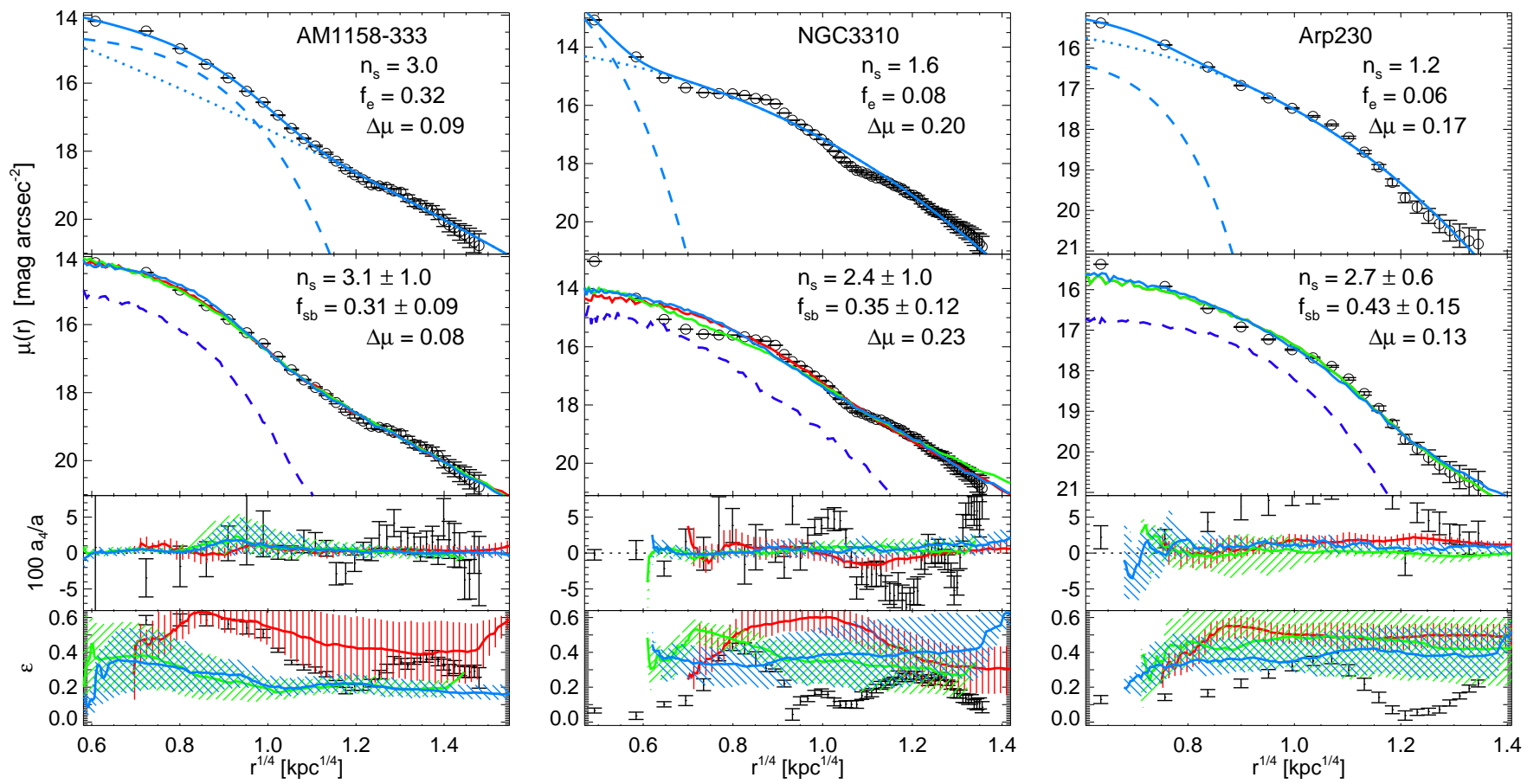

FIG. 37. - Figure 21 continued. NGC3310 shows a face-on ring and arm structure at $\sim 1 \mathrm{kpc}$, pushing the simulated extra light component to larger radii.

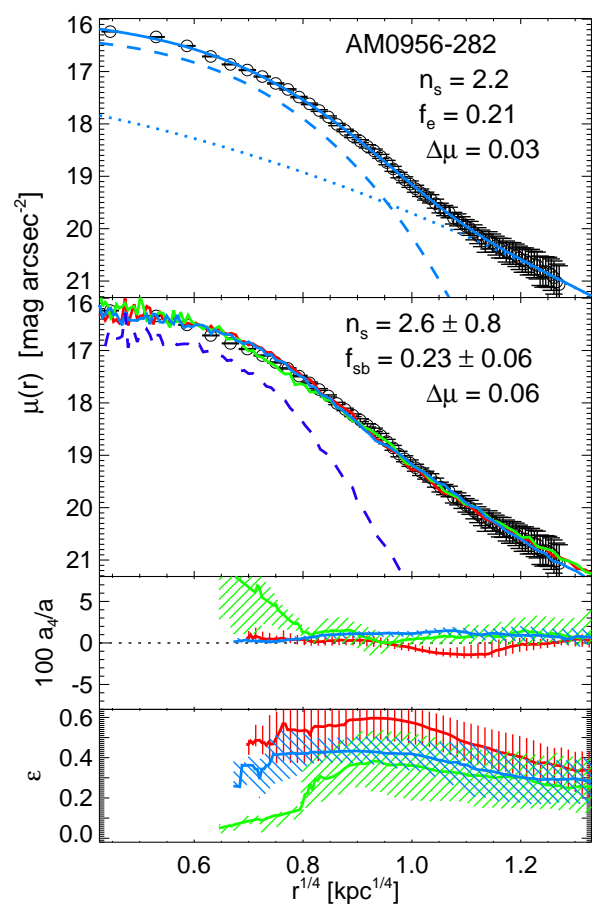

FIG. 38.- Figure21 continued. 
TABLE 1

Fits to RJ04 MERGER REMNANTS

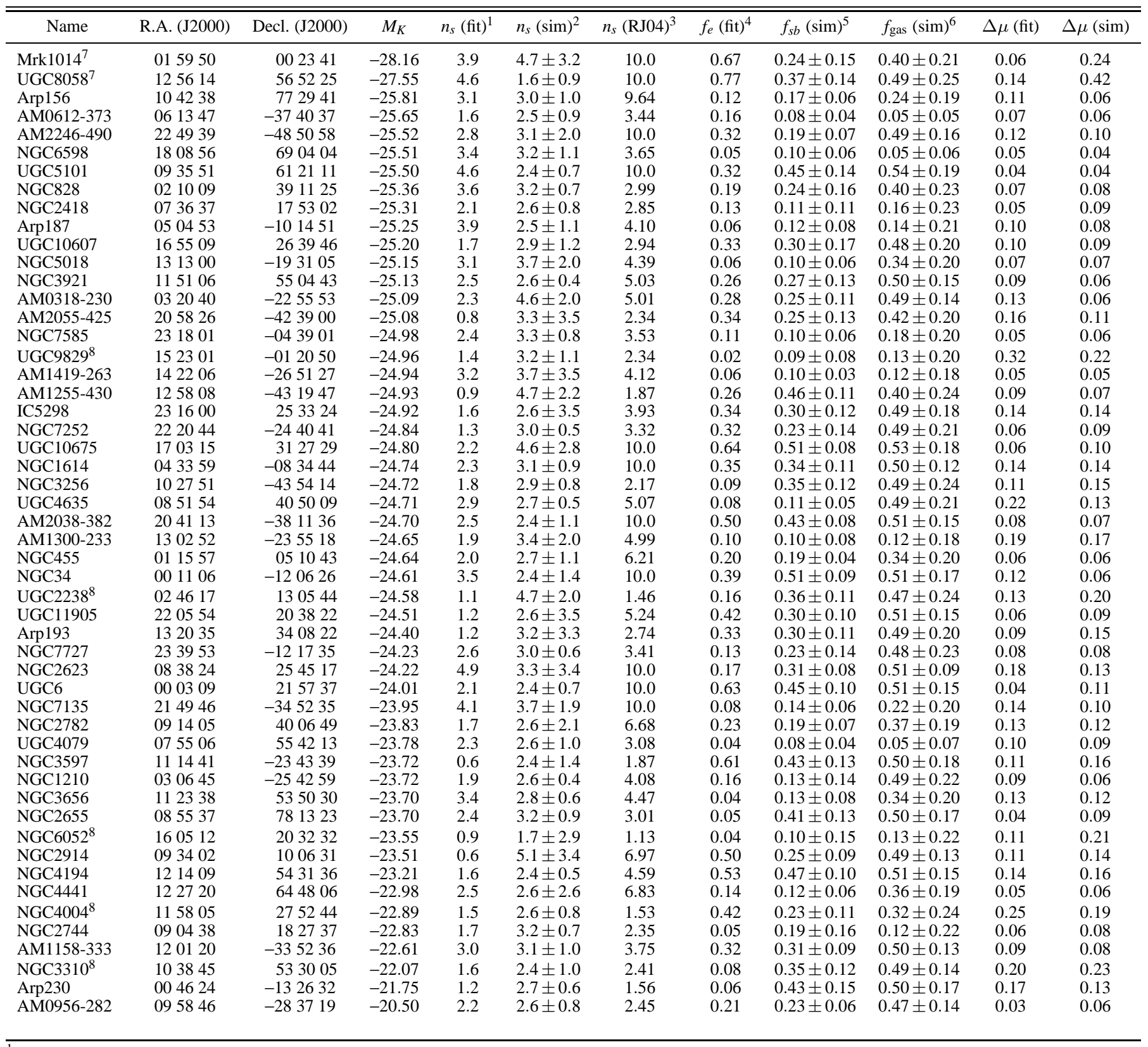

${ }^{1}$ Outer Sersic index $n_{s}$ of the two-component best-fit profile.

2 Range of outer Sersic indices fit in the same manner to the best-fit simulations, at $t \approx 1-3 \mathrm{Gyr}$ after the merger when the system has relaxed.

${ }^{3}$ Sersic index fit to the entire profile (i.e. not decomposed into an outer Sersic and inner extra light component) in RJ04. Note the authors impose a maximum $n_{s}=10.0$.

4 Fraction of light in the inner or "extra light" component of the fits.

${ }^{5}$ Fraction of light from stars produced in the central, merger-induced starburst in the best-fit simulations ( \pm the approximate interquartile range allowed). Note that this may be biased to high values for actively star-forming (especially LIRG and ULIRG) systems.

${ }^{6}$ Initial gas fraction in the best-fitting simulations - this is less robust (note the large interquartile range), representing a rough gas fraction of the systems $\sim$ a few Gyr before the final merger, if they evolved in isolation.

7 AGN contamination affects the central regions (i.e. estimated extra light) and prevents a good simulation fit (we only model the stellar profile here).

${ }^{8}$ Unrelaxed or prominent disk/bar features make the comparison with these objects uncertain. 